\title{
How can a Random Phenomenon between Particles be Synchronized Instantaneously and Independently of the Distance Between Said Particles?
}

Mario Mastriani ( $\square$ mmastria@fiu.edu )

Florida International University https://orcid.org/0000-0002-5627-3935

\section{Research Article}

Keywords: entanglement, quantum communication, quantum measurement, randomness.

Posted Date: October 20th, 2021

DOl: https://doi.org/10.21203/rs.3.rs-890985/v1

License: (c) (i) This work is licensed under a Creative Commons Attribution 4.0 International License.

Read Full License 


\section{How can a Random Phenomenon between Particles be Synchronized Instantaneously and Independently of the Distance Between Said Particles?}

Mario Mastriani*

Knight Foundation School of Computing \& Information Sciences, Florida International University, 11200 S.W. 8th st, Miami, FL 33199, USA

E-mail: mmastria@fiu.edu

Keywords: entanglement, quantum communication, quantum measurement, randomness.

Entanglement is a random phenomenon that is instantly synchronized, regardless of the space that mediates between entangled particles. However, the instantaneous transmission of information using entanglement is impossible. This is because the instantaneity in the synchronization of nonlocal outcomes as a consequence of quantum measurement (after the distribution of the entangled pairs) cannot be used for an entanglement-based communication system to transmit information instantaneously. This impossibility stems from the following two reasons: a) the difficulty of controlling non-local outcomes through local actions without the intervention of an auxiliary channel (classical), and b) regardless of the previous point, no communication system based on entanglement can be instantaneous due to the distribution of an entangled pair at relativistic speeds, necessary to generate the quantum channel, each time a qubit must be transmitted. Three simple experiments help to clarify this controversial point. In fact, this study establishes what is truly responsible for the impossibility to transmit information instantaneously of any communication system based on entanglement. In this respect, functional models of the internal behavior of quantum measurement, and entanglement were developed, which allow analyzing the instantaneity post-distribution of entangled particles, before and after a quantum measurement, as well as the randomness in the results obtained from a quantum measurement of the entanglement. In this sense, this study establishes a debate about three possible responsible for the aforementioned randomness: the quantum measurement itself, entanglement, and the human intervention. Finally, homology between the entanglement and the double-slit experiment is presented. 


\section{Introduction}

Over the last hundred years, entanglement ${ }^{[1-3]}$ has become the cornerstone between two of the main pillars of Physics: Special Relativity ${ }^{[4]}$, and Quantum Mechanics. ${ }^{[5]}$ Interestingly, the notion of entanglement arises in 1935 from an article written by Albert Einstein, Boris Podolsky, and Nathan $\operatorname{Rosen}^{[6]}$, where the authors present entanglement as a physical impossibility which would give rise to faster than light manifestations ${ }^{[7]}$, incompatible with Special Relativity. ${ }^{[4]}$ In fact, Einstein used to refer to entanglement as a "spooky action at a distance" ${ }^{,[6-8]}$, by virtue of its apparent violation of local realism. ${ }^{[7,8]}$ It was not until 1964 that the first contribution appeared trying to resolve the controversy about the non-locality of entanglement. It is an article written by John S. Bell ${ }^{[9]}$ where the author proposes an inequality associated with an experiment, which attempts to establish whether entanglement is local in nature or not. Later, John Clauser, Michael Horne, Abner Shimony, and Richard Holt (CHSH) present an improved version of Bell's method called CHSH inequalities. ${ }^{[10]}$ But it was not until 1982 that the first experiment using the aforementioned inequalities was carried out by Alan Aspect ${ }^{[11]}$, an experiment that was repeated the same year using time-varying analyzers. ${ }^{[12]}$ A complete reflection on these experiments in relation to the controversy around the non-locality of the entanglement can be seen in a 2007 Aspect's paper. ${ }^{[13]}$ However, critics have noted these experiments contained "loopholes", which prevented a definitive answer to this question. This was apparently solved in 2015 when Ronald Hanson at Delft University performed what has been called the first loophole-free experiment. ${ }^{[14]}$

On the other hand, and addressing the instantaneous information transfer as the central axis of the discussion, the creators of the teleportation protocol ${ }^{[15]}$ in the first lines of their paper's Introduction, literally, say:

The existence of long range correlations between Einstein-Podolsky-Rosen $(E P R)^{[6]}$ pairs of particles raises the question of their use for information transfer. Einstein himself used the word "telepathically" in this context. ${ }^{[7]}$ It is known that instantaneous information transfer is 
definitely impossible. ${ }^{[8]}$ Here, we show that EPR correlations can nevertheless assist in the "teleportation" of an intact quantum state from one place to another, by a sender who knows neither the state to be teleported nor the location of the intended receiver.

As we can see, although the problem of instantaneity is addressed as well as the impossibility of having it in the teleportation of arbitrary qubits ${ }^{[16,17]}$, this impossibility is incompletely characterized in the literature. Therefore, to begin with our analysis we must make it clear that any communication system based on entanglement implies, sine qua non condition, taking into account the following aspects:

1. entanglement is not a communication system in itself, since it requires,

2. the prior distribution of an entangled pair over great distances at relativistic times, and

3. the elimination of each qubit to be transmitted from the transmitter side in order not to violate the No-Cloning Theorem ${ }^{[18]}$, which gives rise to new and consecutive redistributions of new entangled pairs over long distances at relativistic times.

The literature ${ }^{[19,20]}$ addresses the discussion about the no instantaneity in the transmission of information through a communication system based on entanglement as a fact that exclusively concerns the post-distribution stage of the entangled pairs, leaving the distribution stage completely aside, which is also responsible for the times involved in interactive two-way transmissions. In fact, if somehow the delay times of the post-distribution of entangled pairs could be eliminated, the communication system would still never be instantaneous because of the distribution times of said pairs. It is clear that any instant information transmission thanks to an entanglement-based communication system is impossible. The first question to ask is: why? If it is because of the reasons stated in the literature ${ }^{[19,20]}$, or if these reasons are too oriented to a particular type of experiment which makes post-distribution instantaneity collide with the No-Cloning Theorem. The second, no less important, question is to establish if there is an experiment, or group of them, that can clarify the problem by eliminating the aforementioned conflict, i.e., eliminating the mentioned concomitance. 
On the other hand, if we distribute a pair of entangled particles of the type `between Alice and Bob, and Alice performs a measurement on her particle, the equiprobable outcomes obtained in both ends can only be $|00\rangle$ or $|11\rangle$. In other words, by choosing the Bell state $\left|\beta_{00}\right\rangle$ Alice and Bob are performing a quasi-control of the experiment consisting of both obtaining the same result, whichever of the two is possible. In any case, this could not be considered as a key exchange system as it is extremely weak from the point of view of Information security.

Regardless of whether Alice and Bob can take advantage of said communication resulting from a simple measurement to send information instantaneously, there has been a synchronization with a delay equal to zero in the outcomes obtained by both. On the other hand, in this experiment, it is not necessary to use a classic disambiguation channel as it is in the quantum teleportation protocol ${ }^{[15]}$, and even so the post-distribution instantaneity does not collide with the No-Cloning Theorem as it does in the experiment of the literature. ${ }^{[19,20]}$ Based on this reasoning, we wonder if a little more control is possible over the experiment, i.e., over both outcomes, since it seems that it all comes down to control, due to the fact that nature clearly makes use of the instantaneity in the synchronization of local and non-local outcomes without seeming to care much about a conflict with the No-Cloning Theorem.

Based on the above, we establish the following hypothesis: There is at least one protocol that does not put post-distribution instantaneity on a collision course with the No-Cloning Theorem. A confirmation of this hypothesis would imply the fact that it is possible to control non-local outcomes through local actions, eliminating the post-distribution transmission times, which does not represent at all an instantaneous transmission of information by a communication system that uses entanglement.

The quantum teleportation protocol ${ }^{[15]}$ is a clear example that the control mentioned in the previous hypothesis is currently only achieved through a classical disambiguating channel, in which the disambiguation bits travel long distances at relativistic times. Therefore, between the 
distribution times of the entangled pairs and the travel times of the disambiguation bits through the classical channel, the only thing that justifies the use of entanglement-based quantum communication protocols compared to their classical counterparts are aspects related to security through quantum cryptography protocols ${ }^{[21,22]}$ with a clear projection on the future quantum Internet. $^{[23-36]}$

The above hypothesis represents the central idea of this paper, in addition to clarifying concepts such as: a) instantaneity of the effect vs instantaneity of the communication system, b) control of non-local outcomes through local actions, i.e., control of entanglement, and c) a new setup for modeling quantum measurement on a given basis.

The outline of the paper is as follows. In Section 2, the concepts of quantum measurement of entangled particles are introduced. Section 3 analyses the quasi-control of non-local outcomes via local actions, while Section 4 analysis the control of non-local outcomes via local actions. In Section 5, we extend this analysis to the quantum teleportation protocol. Section 6 presents the interpretation of the results of the three previous experiments, and direct consequences of the model. The conclusions are presented in Section 7.

\section{Measuring entanglement}

By means of the superposition principle ${ }^{[37]}$, every wave-function can be represented by a point on the Bloch sphere ${ }^{[37]}$,

$|\psi\rangle=\alpha|0\rangle+\beta|1\rangle$

where $\{|0\rangle,|1\rangle\}$ represent the so-called Computational Basis States (CBS) or qubit basis states, which are located at the poles of the already mentioned sphere,

$$
\begin{aligned}
& \text { Spin up }=|H\rangle=|0\rangle=\left[\begin{array}{l}
1 \\
0
\end{array}\right]=\text { North pole, } \\
& \text { Spin down }=|V\rangle=|1\rangle=\left[\begin{array}{l}
0 \\
1
\end{array}\right]=\text { South pole, }
\end{aligned}
$$


and $\{|H\rangle,|V\rangle\}$ means horizontal and vertical polarized states, respectively. Besides, $\{\alpha, \beta\} \in \mathfrak{E}$ represent the projections onto the mentioned CBS, such that $|\alpha|^{2}+|\beta|^{2}=1$. On the other hand, in quantum information processing ${ }^{[37]}$, we can perform measurements on three types of bases ${ }^{[38]}$. $\{|0\rangle,|1\rangle\}=\{|0\rangle, X|0\rangle\}=\{|H\rangle,|V\rangle\}$

$\{|+\rangle,|-\rangle\}=\{H|0\rangle, H X|0\rangle\}=\{|D\rangle,|A\rangle\}$,

$\{|+i\rangle,|-i\rangle\}=\{S H|0\rangle, S H X|0\rangle\}=\{|R\rangle,|L\rangle\}$,

where $i=\sqrt{-1}$, and

$|D\rangle=(|H\rangle+|V\rangle) / \sqrt{2}$,

$|A\rangle=(|H\rangle-|V\rangle) / \sqrt{2}$,

$|R\rangle=(|H\rangle+i|V\rangle) / \sqrt{2}$,

$|L\rangle=(|H\rangle-i|V\rangle) / \sqrt{2}$,

$X=\left[\begin{array}{ll}0 & 1 \\ 1 & 0\end{array}\right]$,

$H=\frac{1}{\sqrt{2}}\left[\begin{array}{cc}1 & 1 \\ 1 & -1\end{array}\right]$, and

$S=\left[\begin{array}{ll}1 & 0 \\ 0 & i\end{array}\right]$.

For the three experiments performed in this study, we will work exclusively with the $\{|H\rangle,|V\rangle\}$, basis for which we will define two operators known as the projectors $P_{H}$ and $P_{V}$ onto the states $|H\rangle$ and $|V\rangle$ respectively, which are represented by their density matrices,

$P_{H}=|0\rangle\langle 0|=| H\rangle\langle H|=\frac{I+Z}{2}=\left[\begin{array}{ll}1 & 0 \\ 0 & 0\end{array}\right]=M_{0}, \quad$ and

$P_{V}=|1\rangle\langle 1|=| V\rangle\langle V|=\frac{I-Z}{2}=\left[\begin{array}{ll}0 & 0 \\ 0 & 1\end{array}\right]=M_{1}$,

where $M_{0}$ and $M_{1}$ are the operators associated to the measurement process also known as Kraus 
operators $^{[37,38]}$, which we will explain later, while

$I=\left[\begin{array}{ll}1 & 0 \\ 0 & 1\end{array}\right]=|0\rangle\langle 0|+| 1\rangle\langle 1|=P_{H}+P_{V}=M_{0}+M_{1}, \quad$ and

$Z=\left[\begin{array}{cc}1 & 0 \\ 0 & -1\end{array}\right]=|0\rangle\langle 0|-| 1\rangle\langle 1|=P_{H}-P_{V}=M_{0}-M_{1}$

The projectors $\left\{P_{H}, P_{V}\right\}$ are known as horizontal and vertical polarizers, respectively, and are widely used in all quantum optics experiments ${ }^{[39]}$, which, as we can see in Equation 16 comply with $P_{H}+P_{V}=I$. In the chosen $\{|H\rangle,|V\rangle\}$ basis, the projectors $\left\{P_{H}, P_{V}\right\}$ also constitute a basis, $M=\left\{P_{H}, P_{V}\right\}=\left\{\left[\begin{array}{ll}1 & 0 \\ 0 & 0\end{array}\right],\left[\begin{array}{ll}0 & 0 \\ 0 & 1\end{array}\right]\right\}$,

where $M$ is the measurement operator, which is not a unitary operator that collapses the wavefunction in all cases with the exception of the CBS. This last example, in particular, will be explained later. Besides, quantum measurement procedure is an absolutely random process, in such a way that when applying this operator on a generic qubit, like that of Equation 1, we obtain the following equation,

$\left.M|\psi\rangle=\left\{P_{H}|\psi\rangle, P_{V}|\psi\rangle\right\}=\left\{\left[\begin{array}{ll}1 & 0 \\ 0 & 0\end{array}\right]\left[\begin{array}{l}\alpha \\ \beta\end{array}\right],\left[\begin{array}{ll}0 & 0 \\ 0 & 1\end{array}\right]\left[\begin{array}{l}\alpha \\ \beta\end{array}\right]\right\}=\left\{\begin{array}{c}\alpha \\ 0\end{array}\right],\left[\begin{array}{l}0 \\ \beta\end{array}\right]\right\}$,

whose associated probabilities are $\left\{P_{0}, P_{1}\right\}$, where $P_{0}$ represents the projection of $|\psi\rangle$ onto $|0\rangle$, while $P_{1}$ represents the projection of $|\psi\rangle$ onto $|1\rangle$, that is, on the $\{|H\rangle,|V\rangle\}$ basis, being,

$P_{0}=\left\langle\psi^{\dagger}\left|M_{0}^{\dagger} M_{0}\right| \psi\right\rangle$, and

$P_{1}=\left\langle\psi^{\dagger}\left|M_{1}^{\dagger} M_{1}\right| \psi\right\rangle$,

where $(\bullet)^{\dagger}$ denotes transposition and complex conjugate of $(\bullet)$. It is evident that operator $M$ alters what is measured, except in the case of CBS $\{|0\rangle,|1\rangle\}$, which are unaltered with the measurement. In fact, by applying the $M$ operator on the CBS, we obtain the following in order, 


$$
|0\rangle_{p m}=M|0\rangle=\left\{M_{0}|0\rangle, M_{1}|0\rangle\right\}=\left\{\left[\begin{array}{ll}
1 & 0 \\
0 & 0
\end{array}\right]\left[\begin{array}{l}
1 \\
0
\end{array}\right],\left[\begin{array}{ll}
0 & 0 \\
0 & 1
\end{array}\right]\left[\begin{array}{l}
1 \\
0
\end{array}\right]\right\}=\left\{\left[\begin{array}{l}
1 \\
0
\end{array}\right],\left[\begin{array}{l}
0 \\
0
\end{array}\right]\right\}=\left\{\begin{array}{cc}
|0\rangle, \text { nothing } \\
100 \% & 0 \%
\end{array}\right\},
$$

where $|0\rangle_{p m}$ means $|0\rangle$ post-measurement, with these probabilities,

$$
\begin{aligned}
& P_{0}=\left\langle 0\left|M_{0}^{\dagger} M_{0}\right| 0\right\rangle=\left[\begin{array}{ll}
1 & 0
\end{array}\right]\left[\begin{array}{ll}
1 & 0 \\
0 & 0
\end{array}\right]\left[\begin{array}{ll}
1 & 0 \\
0 & 0
\end{array}\right]\left[\begin{array}{l}
1 \\
0
\end{array}\right]=1, \\
& P_{1}=\left\langle 0\left|M_{1}^{\dagger} M_{1}\right| 0\right\rangle=\left[\begin{array}{ll}
1 & 0
\end{array}\right]\left[\begin{array}{ll}
0 & 0 \\
0 & 1
\end{array}\right]\left[\begin{array}{ll}
0 & 0 \\
0 & 1
\end{array}\right]\left[\begin{array}{l}
1 \\
0
\end{array}\right]=0,
\end{aligned}
$$

and,

$$
|1\rangle_{p m}=M|1\rangle=\left\{M_{0}|1\rangle, M_{1}|1\rangle\right\}=\left\{\left[\begin{array}{ll}
1 & 0 \\
0 & 0
\end{array}\right]\left[\begin{array}{l}
0 \\
1
\end{array}\right],\left[\begin{array}{ll}
0 & 0 \\
0 & 1
\end{array}\right]\left[\begin{array}{l}
0 \\
1
\end{array}\right]\right\}=\left\{\left[\begin{array}{l}
0 \\
0
\end{array}\right],\left[\begin{array}{l}
0 \\
1
\end{array}\right]\right\}=\left\{\begin{array}{cc}
\text { nothing }, & |1\rangle \\
0 \% & 100 \%
\end{array}\right\}
$$

where $|1\rangle_{p m}$ means $|1\rangle$ post-measurement, with these probabilities,

$$
\begin{aligned}
& P_{0}=\left\langle 1\left|M_{0}^{\dagger} M_{0}\right| 1\right\rangle=\left[\begin{array}{ll}
0 & 1
\end{array}\right]\left[\begin{array}{ll}
1 & 0 \\
0 & 0
\end{array}\right]\left[\begin{array}{ll}
1 & 0 \\
0 & 0
\end{array}\right]\left[\begin{array}{l}
0 \\
1
\end{array}\right]=0, \\
& P_{1}=\left\langle 1\left|M_{1}^{\dagger} M_{1}\right| 1\right\rangle=\left[\begin{array}{ll}
0 & 1
\end{array}\right]\left[\begin{array}{ll}
0 & 0 \\
0 & 1
\end{array}\right]\left[\begin{array}{ll}
0 & 0 \\
0 & 1
\end{array}\right]\left[\begin{array}{l}
0 \\
1
\end{array}\right]=1 .
\end{aligned}
$$

That is, both CBS remain unchanged after the measurement.

On the other hand, we are going to build the famous Bell's bases ${ }^{[1-3]}$, with 2-qubit vectors that combine their respective Hilbert spaces $\mathrm{H}^{A \cup B}=\mathrm{H}_{2}^{A} \otimes \mathrm{H}_{2}^{B}$, where the superscripts has to do with Alice and Bob, and then we will have the following four vectors,

$$
\begin{aligned}
& \left|\Phi_{+}^{A \cup B}\right\rangle=\left|\beta_{00}\right\rangle=\frac{1}{\sqrt{2}}\left(\left|0^{A}, 0^{B}\right\rangle+\left|1^{A}, 1^{B}\right\rangle\right), \\
& \left|\Phi_{-}^{A \cup B}\right\rangle=\left|\beta_{10}\right\rangle=\frac{1}{\sqrt{2}}\left(\left|0^{A}, 0^{B}\right\rangle-\left|1^{A}, 1^{B}\right\rangle\right), \\
& \left|\Psi_{+}^{A \cup B}\right\rangle=\left|\beta_{01}\right\rangle=\frac{1}{\sqrt{2}}\left(\left|0^{A}, 1^{B}\right\rangle+\left|1^{A}, 0^{B}\right\rangle\right), \\
& \left|\Psi_{-}^{A \cup B}\right\rangle=\left|\beta_{11}\right\rangle=\frac{1}{\sqrt{2}}\left(\left|0^{A}, 1^{B}\right\rangle-\left|1^{A}, 0^{B}\right\rangle\right) .
\end{aligned}
$$

In this study, we will only work with the first Bell states of Equation 28, i.e., $\left|\beta_{00}\right\rangle$. Then, by applying the measurement operator $M$ to the Bell state $\left|\beta_{00}\right\rangle$ after distributing the entangled pair 
between Alice and Bob, two important options emerge for the experiments present in later sections: a) Alice and Bob measure their respective states simultaneously, or b) One of them measures first, e.g. Alice, who is always associated with the emitter of an eventual communication system in literature. Both options are contemplated in the following subsections.

\subsection{Alice and Bob measure their particles simultaneously}

It is practically impossible for Alice and Bob to measure their particles at the same time according to the configuration of Figure 1, however, the important thing in this case is to correctly define the components of the measurement operator $M$. As we mentioned in Section 1 , there are only two possible equiprobable outcomes for this configuration, each with a $50 \%$ chance of happening: $|00\rangle$ and $|11\rangle$, which can be seen in the probability graph of Figure 1 .

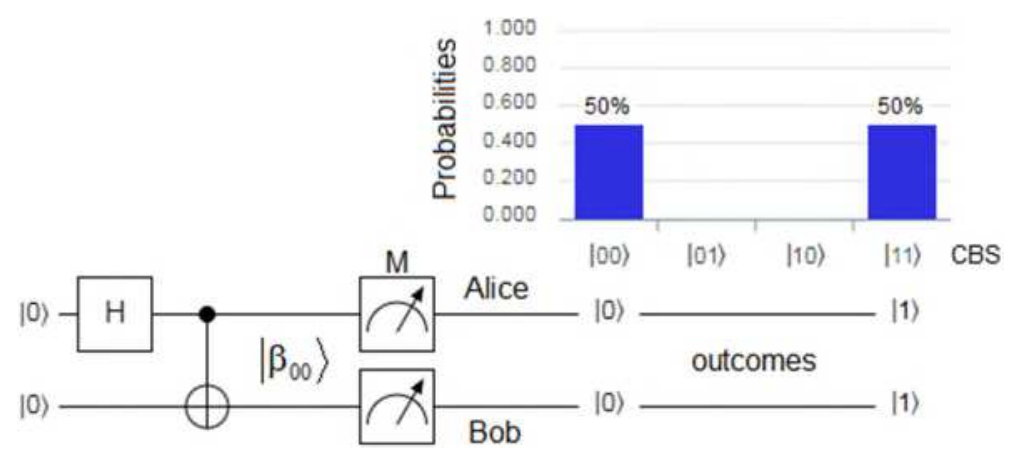

Figure 1. Application of the measurement operator $M$ on an entangled pair. Alice and Bob measure their particles simultaneously.

For this case the components of the measurement operator $M$ will be:

$$
\begin{aligned}
& \Pi_{0}=M_{0} \otimes M_{0}=\left[\begin{array}{ll}
1 & 0 \\
0 & 0
\end{array}\right] \otimes\left[\begin{array}{ll}
1 & 0 \\
0 & 0
\end{array}\right]=\left[\begin{array}{llll}
1 & 0 & 0 & 0 \\
0 & 0 & 0 & 0 \\
0 & 0 & 0 & 0 \\
0 & 0 & 0 & 0
\end{array}\right], \quad \text { and } \\
& \Pi_{1}=M_{1} \otimes M_{1}=\left[\begin{array}{ll}
0 & 0 \\
0 & 1
\end{array}\right] \otimes\left[\begin{array}{ll}
0 & 0 \\
0 & 1
\end{array}\right]=\left[\begin{array}{llll}
0 & 0 & 0 & 0 \\
0 & 0 & 0 & 0 \\
0 & 0 & 0 & 0 \\
0 & 0 & 0 & 1
\end{array}\right]
\end{aligned}
$$

where $\otimes$ represents the Kronecker product. ${ }^{[37]}$ 
Then, applying the measurement operator $M$, defined in terms of $\Pi_{0}$ and $\Pi_{1}$, to $\left|\beta_{00}\right\rangle$, yields,

$$
\begin{aligned}
\left|\beta_{00}\right\rangle_{p m} & =M\left|\beta_{00}\right\rangle=\left\{\Pi_{0}\left|\beta_{00}\right\rangle, \Pi_{1}\left|\beta_{00}\right\rangle\right\} \\
& =\left\{\left[\begin{array}{cccc}
1 & 0 & 0 & 0 \\
0 & 0 & 0 & 0 \\
0 & 0 & 0 & 0 \\
0 & 0 & 0 & 0
\end{array}\right]\left[\begin{array}{c}
1 / \sqrt{2} \\
0 \\
0 \\
1 / \sqrt{2}
\end{array}\right],\left[\begin{array}{cccc}
0 & 0 & 0 & 0 \\
0 & 0 & 0 & 0 \\
0 & 0 & 0 & 0 \\
0 & 0 & 0 & 1
\end{array}\right]\left[\begin{array}{c}
1 / \sqrt{2} \\
0 \\
0 \\
1 / \sqrt{2}
\end{array}\right]\right\} \\
& =\left\{\left[\begin{array}{c}
1 / \sqrt{2} \\
0 \\
0 \\
0
\end{array}\right],\left[\begin{array}{c}
0 \\
0 \\
0 \\
1 / \sqrt{2}
\end{array}\right]\right\}=\left\{\begin{array}{rc}
|00\rangle / \sqrt{2},|00\rangle / \sqrt{2} \\
50 \% & 50 \% \\
\text { head } & \text { tail } \\
(\equiv 0) & (\equiv 1)
\end{array}\right\},
\end{aligned}
$$

with these probabilities,

$$
\begin{gathered}
P_{0}=\left\langle\beta_{00}\left|\Pi_{0}^{\dagger} \Pi_{0}\right| \beta_{00}\right\rangle=\left[\begin{array}{llll}
1 / \sqrt{2} & 0 & 0 & 1 / \sqrt{2}
\end{array}\right]\left[\begin{array}{llll}
1 & 0 & 0 & 0 \\
0 & 0 & 0 & 0 \\
0 & 0 & 0 & 0 \\
0 & 0 & 0 & 0
\end{array}\right]\left[\begin{array}{cccc}
1 & 0 & 0 & 0 \\
0 & 0 & 0 & 0 \\
0 & 0 & 0 & 0 \\
0 & 0 & 0 & 0
\end{array}\right]\left[\begin{array}{c}
1 / \sqrt{2} \\
0 \\
0 \\
1 / \sqrt{2}
\end{array}\right]=\frac{1}{2}, \\
P_{1}=\left\langle\beta_{00}\left|\Pi_{1}^{\dagger} \Pi_{1}\right| \beta_{00}\right\rangle=\left[\begin{array}{llll}
1 / \sqrt{2} & 0 & 0 & 1 / \sqrt{2}
\end{array}\right]\left[\begin{array}{llll}
0 & 0 & 0 & 0 \\
0 & 0 & 0 & 0 \\
0 & 0 & 0 & 0 \\
0 & 0 & 0 & 1
\end{array}\right]\left[\begin{array}{llll}
0 & 0 & 0 & 0 \\
0 & 0 & 0 & 0 \\
0 & 0 & 0 & 0 \\
0 & 0 & 0 & 1
\end{array}\right]\left[\begin{array}{c}
1 / \sqrt{2} \\
0 \\
0 \\
1 / \sqrt{2}
\end{array}\right]=\frac{1}{2} .
\end{gathered}
$$

By applying the measurement operator $M$ to the Bell state $\left|\beta_{00}\right\rangle$, the outcomes obtained can be associated with the result of tossing a coin, whose values will be equiprobable, i.e., $50 \%$ getting head, which we can interpret in binary form as zero ( head $\equiv 0)$, and $50 \%$ getting tail, the latter associated in a binary way with a one $($ tail $\equiv 1)$, which we can clearly see in the last member of the Equation 31. In other words, it is a space of 2 equiprobable results considering the chosen basis $\{|H\rangle,|V\rangle\}$ and the qubit to which the measurement operator $M$ is applied, i.e., $\left|\beta_{00}\right\rangle$.

\subsection{Alice measures her particle first}

Based on Figure 2, we must define the constituent components of the measurement operator $M$ that we must use in this case, 


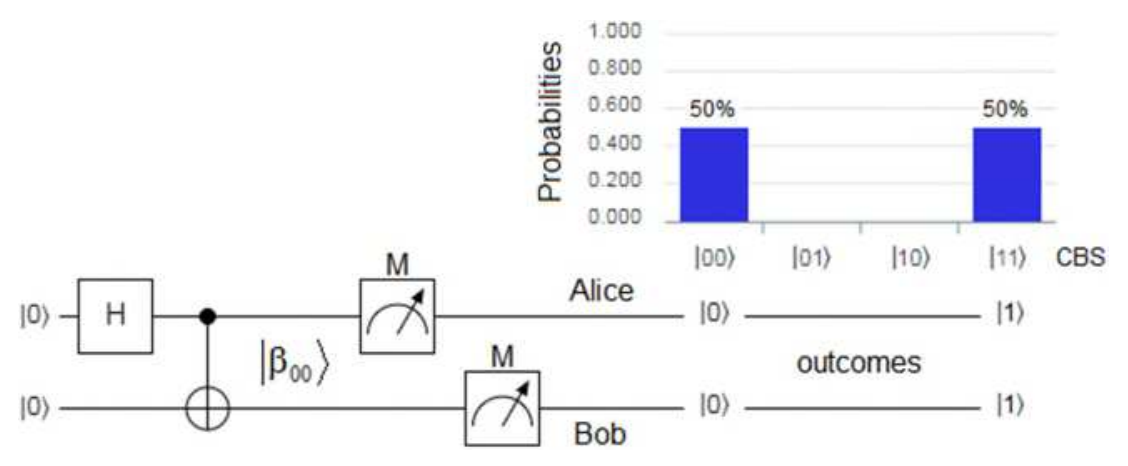

Figure 2. Application of the measurement operator $M$ on an entangled pair. Alice measures her particle first, for an imaginary timeline from left to right of the figure.

$\Pi_{0}=M_{0} \otimes I=\left[\begin{array}{ll}1 & 0 \\ 0 & 0\end{array}\right] \otimes\left[\begin{array}{ll}1 & 0 \\ 0 & 1\end{array}\right]=\left[\begin{array}{llll}1 & 0 & 0 & 0 \\ 0 & 1 & 0 & 0 \\ 0 & 0 & 0 & 0 \\ 0 & 0 & 0 & 0\end{array}\right], \quad$ and

$\Pi_{1}=M_{1} \otimes I=\left[\begin{array}{ll}0 & 0 \\ 0 & 1\end{array}\right] \otimes\left[\begin{array}{ll}1 & 0 \\ 0 & 1\end{array}\right]=\left[\begin{array}{llll}0 & 0 & 0 & 0 \\ 0 & 0 & 0 & 0 \\ 0 & 0 & 1 & 0 \\ 0 & 0 & 0 & 1\end{array}\right]$.

Then, applying the measurement operator $M$, defined in terms of the new projectors $\Pi_{0}$ and $\Pi_{1}$, to $\left|\beta_{00}\right\rangle$, yields,

$$
\begin{aligned}
\left|\beta_{00}\right\rangle_{p m} & =M\left|\beta_{00}\right\rangle=\left\{\Pi_{0}\left|\beta_{00}\right\rangle, \Pi_{1}\left|\beta_{00}\right\rangle\right\} \\
& =\left\{\left[\begin{array}{cccc}
1 & 0 & 0 & 0 \\
0 & 1 & 0 & 0 \\
0 & 0 & 0 & 0 \\
0 & 0 & 0 & 0
\end{array}\right]\left[\begin{array}{c}
1 / \sqrt{2} \\
0 \\
0 \\
1 / \sqrt{2}
\end{array}\right],\left[\begin{array}{cccc}
0 & 0 & 0 & 0 \\
0 & 0 & 0 & 0 \\
0 & 0 & 1 & 0 \\
0 & 0 & 0 & 1
\end{array}\right]\left[\begin{array}{c}
1 / \sqrt{2} \\
0 \\
0 \\
1 / \sqrt{2}
\end{array}\right]\right\} \\
& =\left\{\left[\begin{array}{c}
1 / \sqrt{2} \\
0 \\
0 \\
0
\end{array}\right],\left[\begin{array}{c}
0 \\
0 \\
0 \\
1 / \sqrt{2}
\end{array}\right]\right\}=\left\{\begin{array}{rc}
|00\rangle / \sqrt{2}, & 11\rangle / \sqrt{2} \\
50 \% & 50 \% \\
\text { head } & \text { tail } \\
(\equiv 0) & (\equiv 1)
\end{array}\right\},
\end{aligned}
$$

with these probabilities,

$$
P_{0}=\left\langle\beta_{00}\left|\Pi_{0}^{\dagger} \Pi_{0}\right| \beta_{00}\right\rangle=\left[\begin{array}{llll}
1 / \sqrt{2} & 0 & 0 & 1 / \sqrt{2}
\end{array}\right]\left[\begin{array}{cccc}
1 & 0 & 0 & 0 \\
0 & 1 & 0 & 0 \\
0 & 0 & 0 & 0 \\
0 & 0 & 0 & 0
\end{array}\right]\left[\begin{array}{cccc}
1 & 0 & 0 & 0 \\
0 & 1 & 0 & 0 \\
0 & 0 & 0 & 0 \\
0 & 0 & 0 & 0
\end{array}\right]\left[\begin{array}{c}
1 / \sqrt{2} \\
0 \\
0 \\
1 / \sqrt{2}
\end{array}\right]=\frac{1}{2},
$$




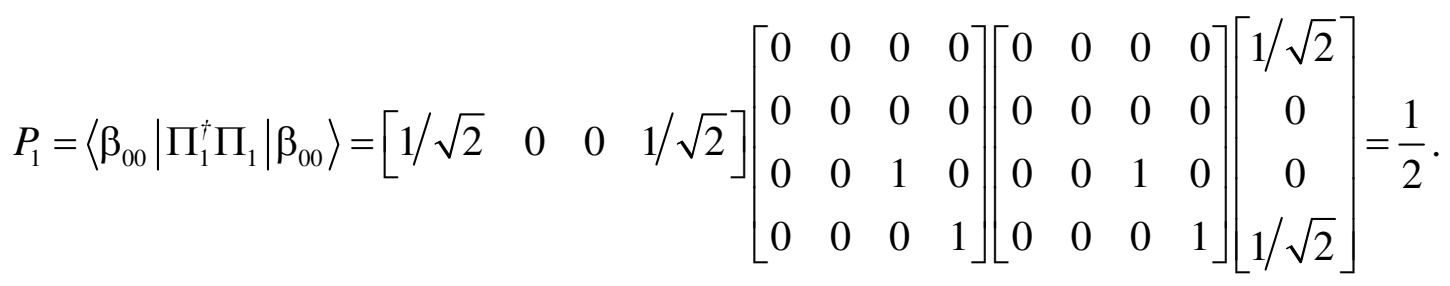

Probabilities obtained in Equations 37 and 38 can be seen below the last member of Equation 36, where, $50 \%$ in each case can also be associated with the result of tossing a coin.

If now Bob measures his particle, said measurement is carried out on a qubit of the type $|0\rangle$ or $|1\rangle$, i.e., on a CBS, which is absolutely identical to Alice's qubit, although completely independent after the collapse of the entanglement due to the measurement carried out by her. In that case, we have to repeat Equations 22 to 27 of this section for the case of a CBS $\{|0\rangle,|1\rangle\}$. Therefore, when Bob measures after Alice, everything will remain the same due to the immunity of the CBS (resulting from both figures) to the quantum measurement process, as Equations 22 and 25 indicate, since in that case they are two mutually independent qubits. In other words, everything relevant to Figures 1 and 2 concludes with the equality between Equations 31, and 36, since it is evident that the final results associated with them are identical. However, as we have already mentioned before, the measurement operator $M$ is non-unitary, provoking the collapse of the entanglement in both branches where entangled particles were distributed regardless the order in which the measurement operator $M$ was applied.

Strictly speaking, both Alice and Bob have to measure, what this comparative analysis tells us is that one of them will inevitably do it before the other. The first to do so will define both outcomes although it is indistinct who measures first. Therefore, as we have mentioned before, in the following experiments we will assume that the one who measures first is always Alice, i.e., she will first collapse the entanglement. In this way, we are consistent with the literature, which has historically assigned Alice the role of emitter. This is of particular interest to the analysis of Section 4.

Finally, Figure 3 presents the implementation of the measurement operator $M$, which is 
commonly used in quantum optics experiments. ${ }^{[39]}$ A polarizer beam-splitter (PBS) allows us to measure a photon on the basis $\{|H\rangle,|V\rangle\} \cdot{ }^{[40,41]}$ This optical element transmits horizontally polarized photons and reflects vertically polarized photons, and an eventual unpolarized photon can collapse to either a horizontal or vertical polarization with equal probability. The purpose of the fiber coupler (FC) of Figure 3 is just to bring these photons to the single photon detectors (SPDs), and thus it can be done via multi-mode fibers (MMF). ${ }^{[40]}$

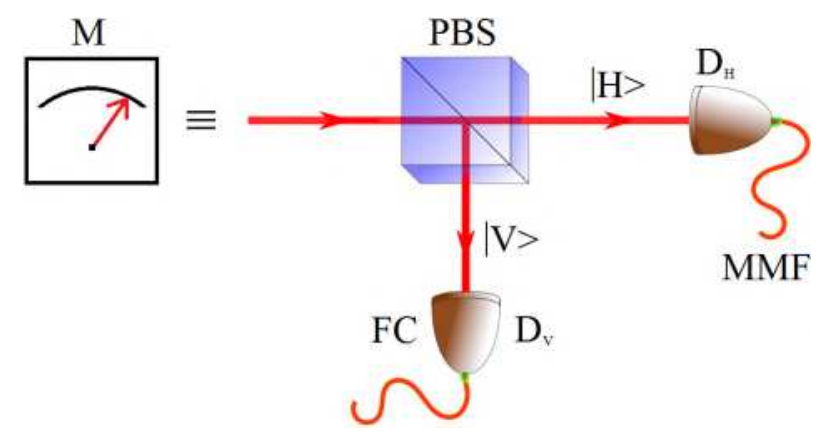

Figure 3. Optical implementation of the measurement operator $M$, where PBS represents a polarization beam-splitter cube, FC means fiber coupler, MMF is multi-mode fibers, $\mathrm{D}_{\mathrm{H}}$ is the horizontal detector, while $\mathrm{D}_{\mathrm{V}}$ is the vertical detector.

This implementation will be a key piece in the modeling of the measurement operator $M$ that is developed in Section 3.

\section{First experiment: quasi-controlling the outcomes}

In this section, as well as in the two subsequent ones, the experiments begin with the generation of a pair of entangled photons from the impact of a laser beam, that emits ultra-violet light at a given wavelength $\lambda_{\text {laser }}$, on a crystal of the type $\beta-\mathrm{BaB}_{2} \mathrm{O}_{4}$ (Beta-Barium Borate or $\mathrm{BBO}$ ), as Figure 4 shows. Both entangled photons are generated by spontaneous parametric down conversion (SPDC) in the nonlinear crystal (BBO), which is designed for type-II phase matching ${ }^{[42]}$, where the correlated photons are emitted in two separate cones with perpendicular polarizations, where one is ordinary polarized and the other extraordinary polarized. ${ }^{[42]}$ Both 
cones are not shown in Figure 4 because they are outside the central axis of this work. The entangled photons are emitted along the intersections of the mentioned cones and are noncollinear with the pump beam (i.e., the beam that immediately exits the laser). These new beams resulting from the aforementioned intersections are called signal and idler, and the relationship between their wavelengths and that of the pump is:

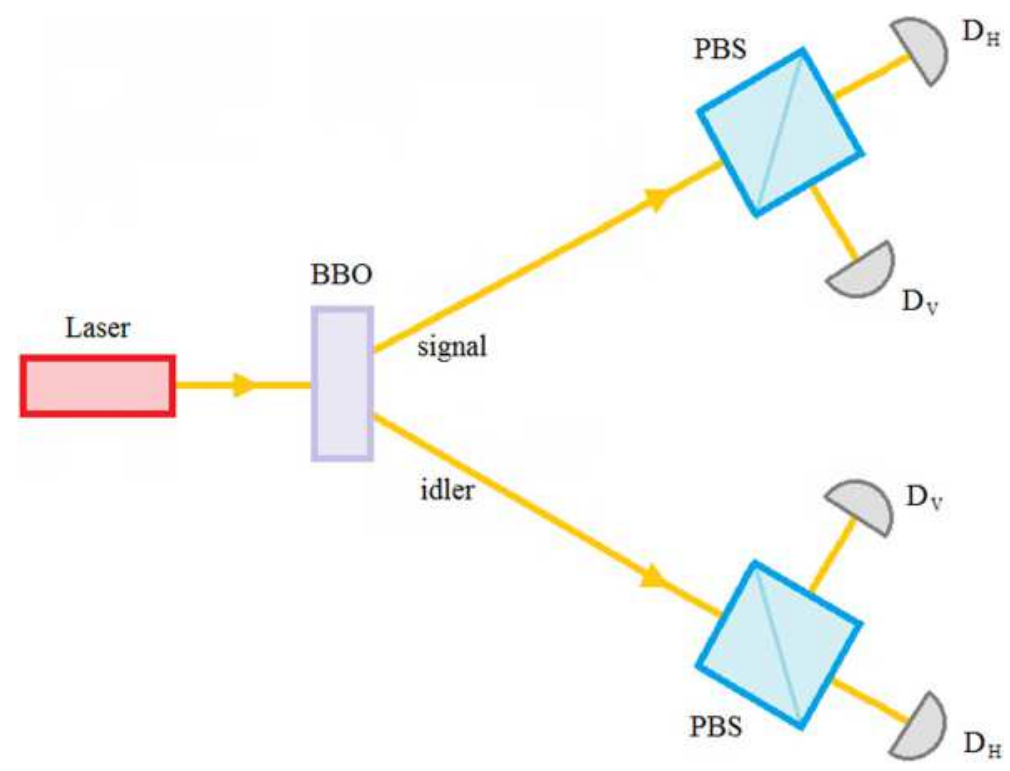

Figure 4. Generation of two entangled photons and their subsequent detection, where BBO means Beta-Barium Borate nonlinear crystal, $\mathrm{PBS}$ is polarizer beam-splitter, and $\left\{\mathrm{D}_{\mathrm{H}}, \mathrm{D}_{\mathrm{V}}\right\}$ are the respective detectors.

$\frac{1}{\lambda_{\text {laser }}}=\frac{1}{\lambda_{\text {signal }}}+\frac{1}{\lambda_{\text {idler }}}$

where $\lambda_{\text {signal }}=\lambda_{\text {idler }}=2 \lambda_{\text {laser }}$, reason why both the signal beam and the idler beam are in the infrared range. At the end of each of the two beams, Figure 4 is completed with both measurements implemented using the PBS and their respective detectors $\left\{D_{H}, D_{V}\right\}$.

Next, we will try to carry out a complete circuit modeling of the configuration of Figure 4, taking into account that Alice and Bob have previously decided to:

1. always measure on the same basis $\{|H\rangle,|V\rangle\}$, and

2. exclusively work with the Bell state $\left|\beta_{00}\right\rangle$. 
With the second condition, Alice and Bob have agreed to have a kind of quasi-control on the result of the experiment, given that, although the result of the entire measurement process is random, said randomness is confined to two possible outcomes: $|00\rangle$, or $|11\rangle$. In other words, Alice and Bob have no idea which of the two results they will get, but they are sure that they will always share the same spins $|0\rangle$, or $|1\rangle$, that is why we speak of quasi-control over the result of the experiment. Moreover, Figure 3 shows that each measurement operator $M$ is composed of a PBS and two detectors, however, from this point on, when we talk about modeling the measurement operator $M$, what we really want to do is modeling the PBS, which we will carry out from the projectors or polarizers $\left\{\mathrm{P}_{\mathrm{H}}, \mathrm{P}_{\mathrm{V}}\right\}$, which has never been implemented in the literature in this way.

The proposed PBS modeling is represented in Figure 5, where said PBS has a Bell state $\left|\beta_{00}\right\rangle$ as input, and one of the equiprobable components of the basis $\{|H\rangle,|V\rangle\}$ as output.

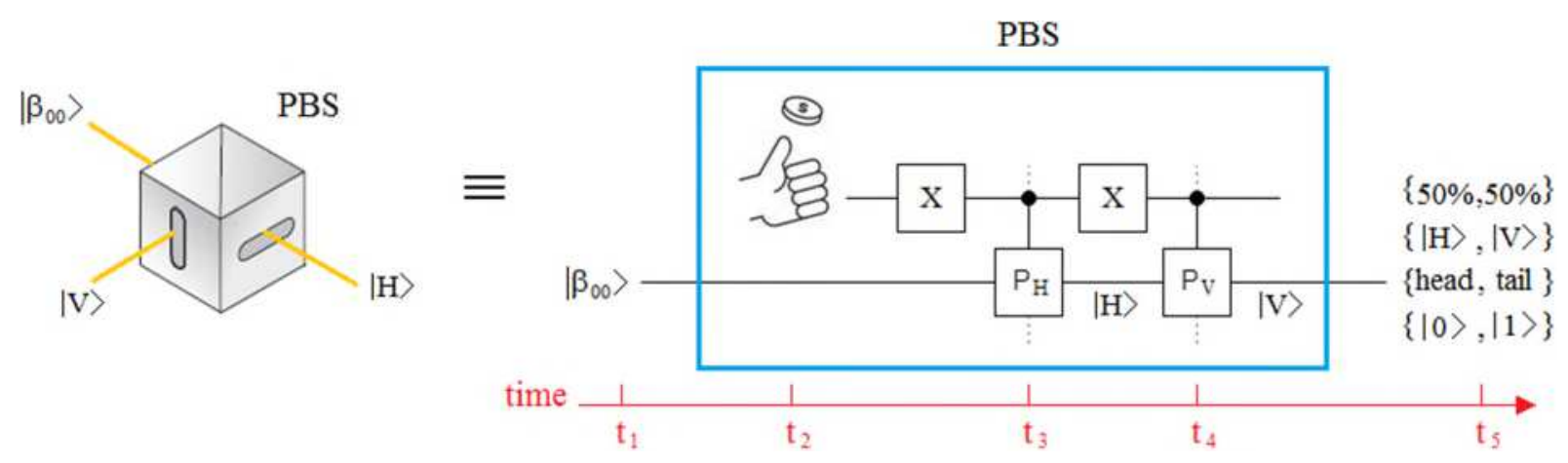

Figure 5. PBS modeling, where $X$ represents the Pauli matrix of Equation (11) which causes a bit-flip on the qubit to which it is applied, while $\left\{\mathrm{P}_{\mathrm{H}}, \mathrm{P}_{\mathrm{V}}\right\}$ are the polarizers onto the $\{|H\rangle,|V\rangle\}$ basis. The outcomes are equiprobable, which is associated to a perfect coin.

This modeling implies two conditions to take into account:

1. It is a closed system, where by closed we must understand that we do not have any possibility of control over the process that takes place inside from the outside, and 
2. we associate the result obtained with the output of the PBS projected onto the $\{|H\rangle,|V\rangle\}$ basis with the result that someone, inside the PBS, tossed a coin in the air, that is, $\{$ head,tail $\} \equiv\{|H\rangle,|V\rangle\} \equiv\{|0\rangle,|1\rangle\}$. This last equivalence has to do with later circuit implementations.

The question is: who flips a coin inside the PBS? Moreover, this is not the only option, in fact, we have three:

- God flips the coin,

- God chooses an outcome, or

- it is a random phenomenon which takes place inside.

Seen from outside, any of the three options is equivalent, therefore, if we associate the first option with the timeline present in the lower part of Figure 5 (red), Table 1 appears.

Table 1. Results obtained inside of the PBS of Figure 5, according to its timeline.

\begin{tabular}{|c|c|c|}
\hline Time & \multicolumn{2}{|c|}{ Result } \\
\hline $\mathrm{t}_{1}$ & \multicolumn{2}{|l|}{ PBS receives to $\left|\beta_{00}\right\rangle$} \\
\hline $\mathrm{t}_{2}$ & \multicolumn{2}{|c|}{$\begin{array}{l}\text { God flips the coin and this result enters to the first } \mathrm{X} \text { gate according to the following } \\
\text { equivalence: }\{\text { head,tail }\} \equiv\{|0\rangle,|1\rangle\} \text {, so that: }\end{array}$} \\
\hline & head $\equiv|0\rangle$ & tail $\equiv|1\rangle$ \\
\hline$t_{3}$ & $\begin{array}{l}X|0\rangle=|1\rangle, \text { then, } \\
\mathrm{P}_{\mathrm{H}} \text { is activated, therefore, }\left|\beta_{00}\right\rangle \text { collapses to } \\
|H\rangle \equiv|0\rangle \text {, i.e., entanglement disappears. }\end{array}$ & $\begin{array}{l}X|1\rangle=|0\rangle \text {, then, } \\
\mathrm{P}_{\mathrm{H}} \text { is not activated }\left(\mathrm{P}_{\mathrm{H}}=\mathrm{I} \text {, identity matrix), }\right. \\
\text { therefore, }\left|\beta_{00}\right\rangle \text { continues to advance, i.e., } \\
\text { entanglement is still active. }\end{array}$ \\
\hline $\mathrm{t}_{4}$ & $\begin{array}{l}X|1\rangle=|0\rangle \text {, then, } \\
\mathrm{P}_{\mathrm{v}} \text { is not activated }\left(\mathrm{P}_{\mathrm{v}}=\mathrm{I} \text {, identity matrix }\right) \text {, } \\
\text { therefore, }|H\rangle \equiv|0\rangle \text { continues to advance } \\
\text { until the exit. }\end{array}$ & $\begin{array}{l}X|0\rangle=|1\rangle, \text { then, } \\
\mathrm{P}_{\mathrm{v}} \text { is activated, therefore, }\left|\beta_{00}\right\rangle \text { collapses to } \\
|V\rangle \equiv|1\rangle \text {, i.e., entanglement disappears. }\end{array}$ \\
\hline $\mathrm{t}_{5}$ & $|H\rangle \equiv|0\rangle \equiv$ head & $|V\rangle \equiv|1\rangle \equiv$ tail \\
\hline
\end{tabular}

Table 1 shows us that the result of tossing a coin inside a PBS is automatically reflected at its exit, when measuring at the basis $\{|H\rangle,|V\rangle\}$ and working with $\left|\beta_{00}\right\rangle$. In consequence, the model of Figure 5 perfectly reflects the behavior of the configuration of Figure 3. 
Now, we will apply the model of Figure 5 to the configuration of Figure 4, which results in

Figure 6, where again we point out that Alice measures first. The application of the PBS to the Alice's beam gives two equiprobable outcomes, in the same way as in Figure 2 in the previous section.

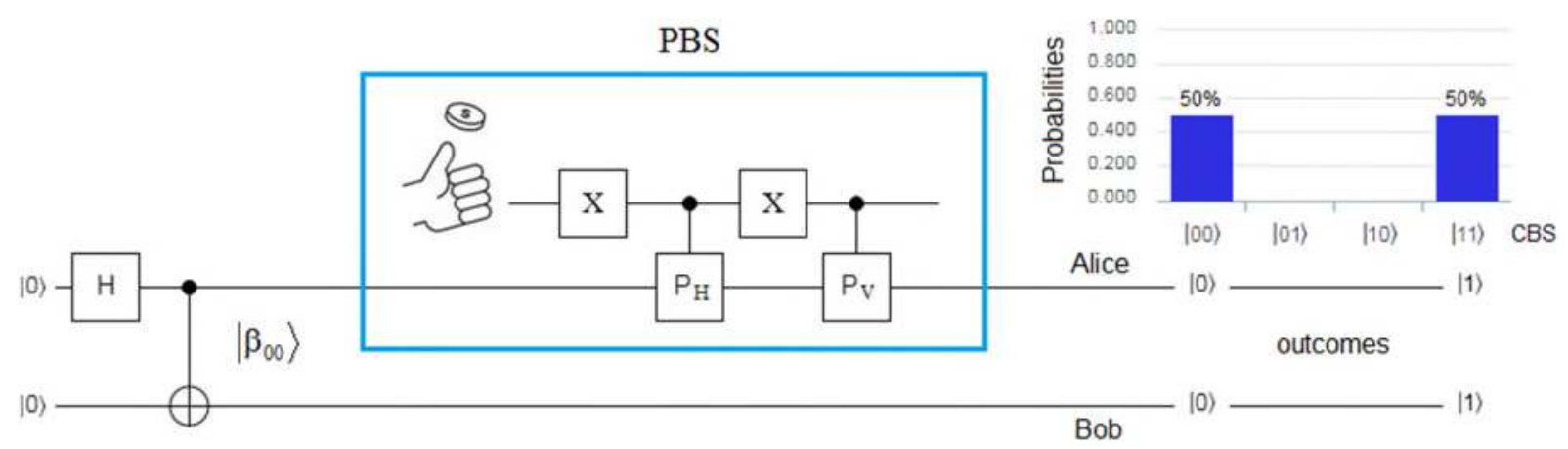

Figure 6. Circuit representation of Figure 4 configuration thanks to the PBS modeling of Figure 5.

Taking into account the conclusions of Table 1, when the result of flipping the coin is head $\equiv|0\rangle, \mathrm{P}_{\mathrm{H}}$ is activated, and $\mathrm{P}_{\mathrm{V}}$ is deactivated, which takes us to a simplified configuration of Figure 6 like that of Figure 7.

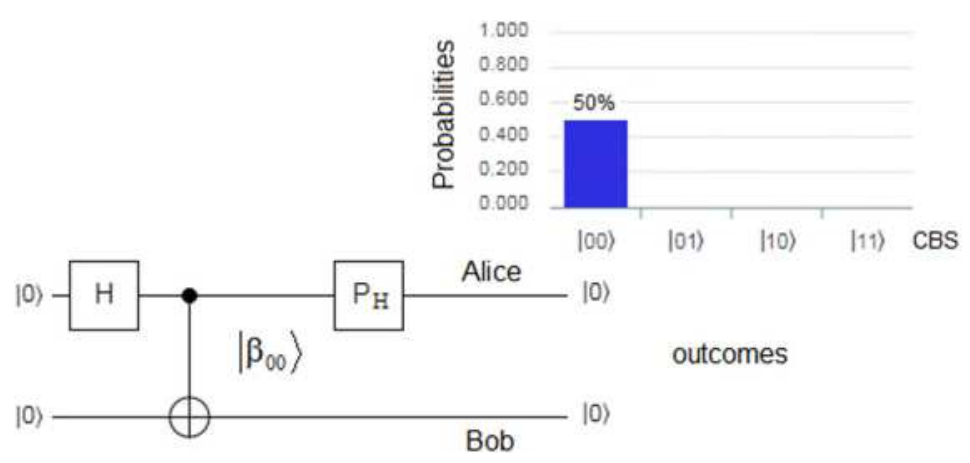

Figure 7. If the result of flipping the coin is head $\equiv|0\rangle$, then, $\mathrm{P}_{\mathrm{H}}$ is only activated.

The result of applying the $\mathrm{P}_{\mathrm{H}}$ in Alice's beam can be calculated as follows,

$$
\left|\beta_{00}\right\rangle_{p m}=\left(P_{H} \otimes I\right)\left|\beta_{00}\right\rangle=\left(\left[\begin{array}{ll}
1 & 0 \\
0 & 0
\end{array}\right] \otimes\left[\begin{array}{ll}
1 & 0 \\
0 & 1
\end{array}\right]\right)\left[\begin{array}{c}
1 / \sqrt{2} \\
0 \\
0 \\
1 / \sqrt{2}
\end{array}\right]=\left[\begin{array}{llll}
1 & 0 & 0 & 0 \\
0 & 1 & 0 & 0 \\
0 & 0 & 0 & 0 \\
0 & 0 & 0 & 0
\end{array}\right]\left[\begin{array}{c}
1 / \sqrt{2} \\
0 \\
0 \\
1 / \sqrt{2}
\end{array}\right]=\left[\begin{array}{c}
1 / \sqrt{2} \\
0 \\
0 \\
0
\end{array}\right]=|00\rangle / \sqrt{2}
$$


with these probabilities,

$$
\begin{aligned}
P_{0} & =\left\langle\beta_{00}\left|\left(P_{H} \otimes I\right)^{\dagger}\left(P_{H} \otimes I\right)\right| \beta_{00}\right\rangle=\left[\begin{array}{llll}
1 / \sqrt{2} & 0 & 0 & 1 / \sqrt{2}
\end{array}\right]\left[\begin{array}{cccc}
1 & 0 & 0 & 0 \\
0 & 1 & 0 & 0 \\
0 & 0 & 0 & 0 \\
0 & 0 & 0 & 0
\end{array}\right]\left[\begin{array}{cccc}
1 & 0 & 0 & 0 \\
0 & 1 & 0 & 0 \\
0 & 0 & 0 & 0 \\
0 & 0 & 0 & 0
\end{array}\right]\left[\begin{array}{c}
1 / \sqrt{2} \\
0 \\
0 \\
1 / \sqrt{2}
\end{array}\right] \\
& =\left[\begin{array}{llll}
1 / \sqrt{2} & 0 & 0 & 0
\end{array}\right]\left[\begin{array}{c}
1 / \sqrt{2} \\
0 \\
0 \\
0
\end{array}\right]=\frac{1}{\sqrt{2}}\left[\begin{array}{llll}
1 & 0 & 0 & 0
\end{array}\right]\left[\begin{array}{c}
1 \\
0 \\
0 \\
0
\end{array}\right] \frac{1}{\sqrt{2}}=\frac{1}{\sqrt{2}}\langle 00 \mid 00\rangle \frac{1}{\sqrt{2}}=\frac{1}{\sqrt{2}} \frac{1}{\sqrt{2}}=\frac{1}{2} .
\end{aligned}
$$

We can see the result of Equation 41 in the upper right margin of Figure 7 (probabilities), which exactly matches what is found in Equation 37, for the first of the two possible results of Equation 36.

Now, when the result of flipping the coin is tail $\equiv|1\rangle, \mathrm{P}_{\mathrm{H}}$ is deactivated, and $\mathrm{P}_{\mathrm{V}}$ is activated, which refers us to a simplified configuration like that of Figure 8.

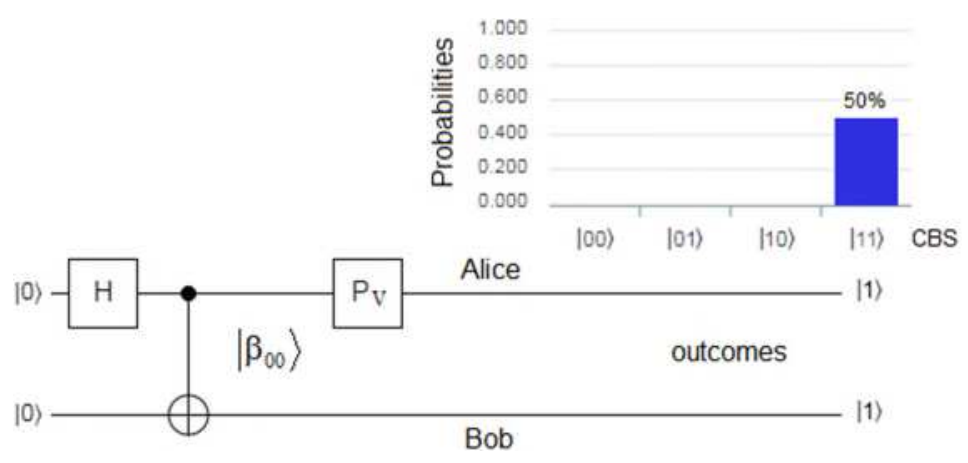

Figure 8. If the result of flipping the coin is tail $\equiv|1\rangle$, then, $\mathrm{P}_{\mathrm{V}}$ is only activated.

The result of applying the $\mathrm{P}_{\mathrm{V}}$ in Alice's beam can be calculated as follows,

$$
\left|\beta_{00}\right\rangle_{p m}=\left(P_{V} \otimes I\right)\left|\beta_{00}\right\rangle=\left(\left[\begin{array}{ll}
0 & 0 \\
0 & 1
\end{array}\right] \otimes\left[\begin{array}{cc}
1 & 0 \\
0 & 1
\end{array}\right]\right)\left[\begin{array}{c}
1 / \sqrt{2} \\
0 \\
0 \\
1 / \sqrt{2}
\end{array}\right]=\left[\begin{array}{llll}
0 & 0 & 0 & 0 \\
0 & 0 & 0 & 0 \\
0 & 0 & 1 & 0 \\
0 & 0 & 0 & 1
\end{array}\right]\left[\begin{array}{c}
1 / \sqrt{2} \\
0 \\
0 \\
1 / \sqrt{2}
\end{array}\right]=\left[\begin{array}{c}
0 \\
0 \\
0 \\
1 / \sqrt{2}
\end{array}\right]=|11\rangle / \sqrt{2}
$$

with these probabilities, 


$$
\begin{aligned}
P_{1} & =\left\langle\beta_{00}\left|\left(P_{V} \otimes I\right)^{\dagger}\left(P_{V} \otimes I\right)\right| \beta_{00}\right\rangle=\left[\begin{array}{llll}
1 / \sqrt{2} & 0 & 0 & 1 / \sqrt{2}
\end{array}\right]\left[\begin{array}{cccc}
0 & 0 & 0 & 0 \\
0 & 0 & 0 & 0 \\
0 & 0 & 1 & 0 \\
0 & 0 & 0 & 1
\end{array}\right]\left[\begin{array}{cccc}
0 & 0 & 0 & 0 \\
0 & 0 & 0 & 0 \\
0 & 0 & 1 & 0 \\
0 & 0 & 0 & 1
\end{array}\right]\left[\begin{array}{c}
1 / \sqrt{2} \\
0 \\
0 \\
1 / \sqrt{2}
\end{array}\right] \\
& =\left[\begin{array}{llll}
0 & 0 & 0 & 1 / \sqrt{2}
\end{array}\right]\left[\begin{array}{c}
0 \\
0 \\
0 \\
1 / \sqrt{2}
\end{array}\right]=\frac{1}{\sqrt{2}}\left[\begin{array}{llll}
0 & 0 & 0 & 1
\end{array}\right]\left[\begin{array}{l}
0 \\
0 \\
0 \\
1
\end{array}\right] \frac{1}{\sqrt{2}}=\frac{1}{\sqrt{2}}\langle 11 \mid 11\rangle \frac{1}{\sqrt{2}}=\frac{1}{\sqrt{2}} \frac{1}{\sqrt{2}}=\frac{1}{2} .
\end{aligned}
$$

Result of Equation 43 is present in the upper right margin of Figure 8 (probabilities), which exactly matches what is found in Equation 38, for the second of the two possible results of Equation 36.

Nature did not use a classical channel to synchronize these results, even then Alice and Bob obtained the same results. In fact, these results were instantly synchronized. In a communication context, the total time of this improvised communication system that allows sharing the orientation of the spin, from the time that the EPR pair distribution between Alice and Bob starts until they obtained their respective outcomes, is:

$T_{t t}=T_{d}+T_{p d}=T_{d} \neq 0$,

where:

- $\quad T_{\mathrm{tt}}$ is the total transmission time, that is, the total time taken by the configuration of Figures 4 and 6 for Alice and Bob to obtain spins with the same orientation,

- $\quad T_{d}$ is the distribution time of the EPR pair, and

- $\quad \mathrm{T}_{\mathrm{pd}}$ is the post-distribution time, i.e., the time it takes to Bob to obtain his outcome after Alice obtains hers after the measurement carried out by her. In other words, the postdistribution time is the synchronization time of Alice's and Bob's outcomes, which is equal to zero.

This analysis tells us that the instantaneity of the synchronization of Alice's and Bob's outcomes has no effect on the total transmission time, which is clearly dominated by the distribution time of the EPR (entangled photons) pair. In other words, no entanglement-based 
communication system can be instantaneous because of the time of distribution of the EPR pair.

Finally, the circuit model of the PBS of Figure 5 fully reflects the behavior of this device, which is of fundamental importance for a comprehensive analysis of entanglement in the context of quantum communication. ${ }^{[24,26,27,43-45]}$

\section{Second experiment: controlling the outcomes}

This section will start with a similar scheme to the first part of Figure 4 in the previous section, that is, with the generation of an entangled pair from the impact of a laser beam on a BBO-type crystal, which gives rise to two new noncollinear beams: signal for Alice, and idler for Bob, see Figure 9. However, and as a fundamental difference with the configuration of Figure 4, we will interpose a rotatable polarizer in the path of Alice's beam, which can only adopt (exclusively) two types of polarizations: horizontal, and vertical, and is controlled by a system consisting of a computer and a Classical-to-Quantum (C2Q) interface, which will convert each bit to its corresponding CBS, i.e., $\{0,1\} \rightarrow[C 2 Q] \rightarrow\{|0\rangle,|1\rangle\}$.

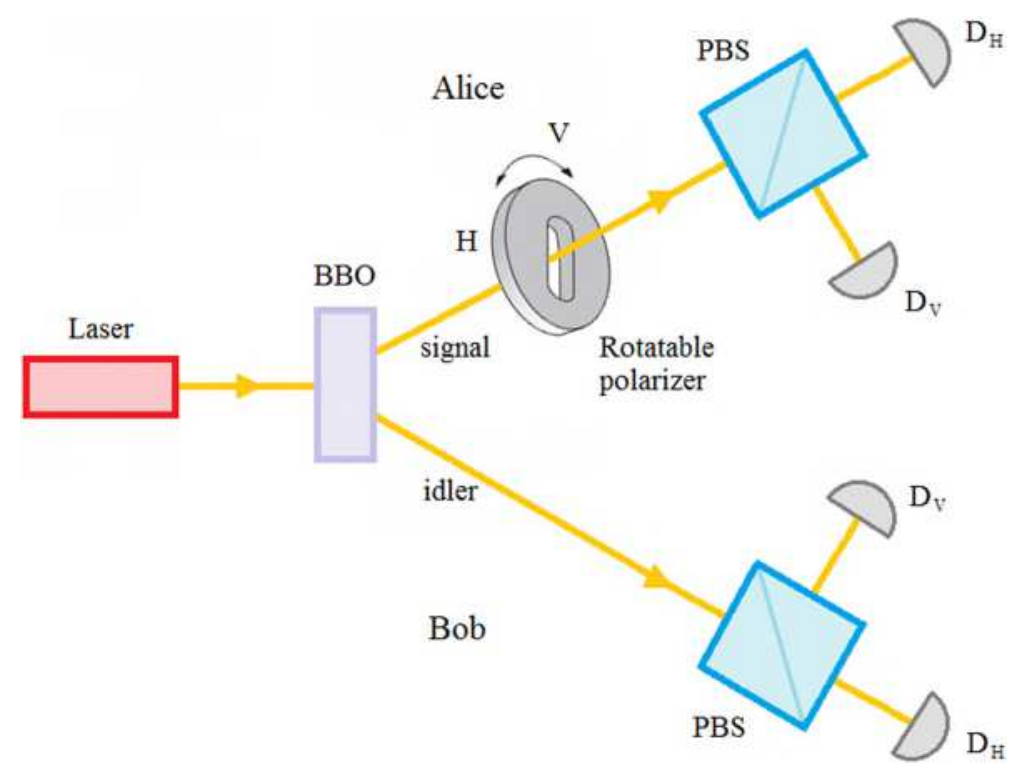

Figure 9. Generation of two entangled photons. Alice applies a rotatable polarizer (with only two options: vertical, and horizontal) controlled by a computer on her beam. Both, Alice and Bob measure. 
In other words, only Alice will use the aforementioned polarizer system, although both, i.e., Alice and Bob will measure. Moreover, in the same way that we did in the previous section, in this experiment we will work with Bell state $\left|\beta_{00}\right\rangle$, and we will measure on the $\{|H\rangle,|V\rangle\}$ basis. Next, we will proceed to model the complete mechanism of the rotatable polarizer with its control computer, and the $\mathrm{C} 2 \mathrm{Q}$ interface. This modeling can be seen in Figure 10. As we can appreciate, this configuration has two fundamental differences with that of Figure 5 for the PBS modeling:

1. while the model on the right side of Figure 5 is a closed system without any possibility of external intervention, the model on the right side of Figure 10, which models the rotatable polarizer, is an open system, i.e., absolutely accessible from the outside, and

2. in the model of Figure 10 we have replaced the toss of a coin with a computer (and a C2Q interface) that generates bits (message) with which we will intend to control the outcomes obtained by Alice and Bob.

In other words, the models in Figures 5 and 10 are identical, changing only two things:

- the accessibility to said models (Figure 5 closed, Figure 10 open), with which we try to have complete control over the outcomes obtained at the end of each beam, and

- the generating entity of the results, which in this case (Figure 10) takes the form of a computer, while in the case of Figure 5 it was about God tossing a coin.

In fact, we have several options when it comes to generating the results (outcomes) in the present experiment:

a) we flip a coin,

b) we choose a value at will,

c) we use a computer that generates random numbers, or

d) the computer gives us a bitstream, which can be a message to be transmitted with an appropriate protocol. 
Respect to the last option, Figure 10 allows us to associate the control of the angle of a rotatable polarizer $\left(0^{\circ} \equiv \mathrm{H}, 90^{\circ} \equiv \mathrm{V}\right)$, thanks to the intervention of an electric motor (absent in Figure 10) controlled by a computer, with an identical circuit configuration to that located inside the blue rectangle of Figure 5, also powered by a computer that delivers the bits associated with the activation of the respective polarizer: $\mathrm{P}_{\mathrm{H}}$, or $\mathrm{P}_{\mathrm{V}}$.
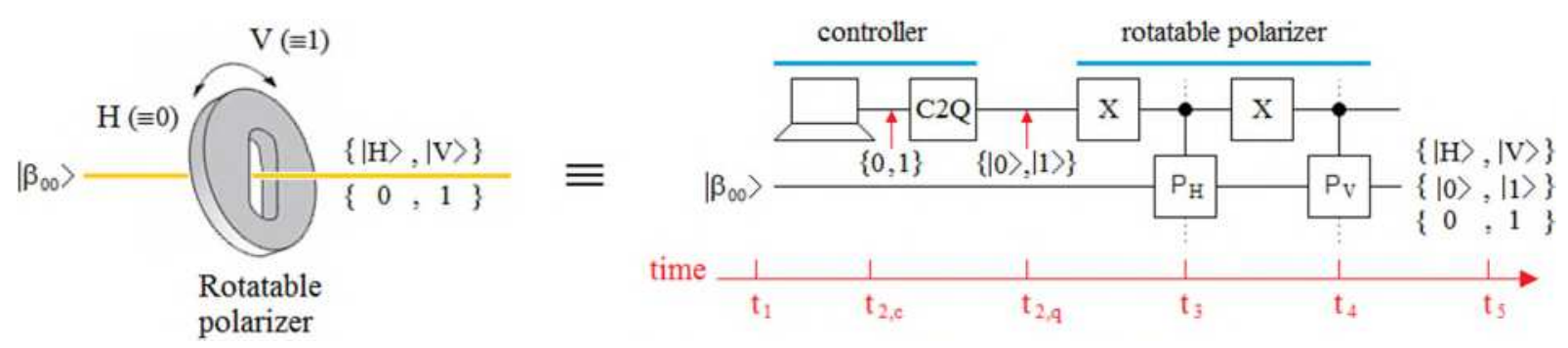

Figure 10. Circuit model of a rotatable polarizer, which can only acquire two polarizations: horizontal, and vertical, according to the bits emitted by the computer.

Developing the time analysis of the model of Figure 10 based on the timeline (red) present below the controller and the rotatable polarizer, we will obtain the results of Table 2.

Table 2. Results obtained from Figure 10, according to its timeline.

\begin{tabular}{|c|c|c|}
\hline Time & \multicolumn{2}{|c|}{ Result } \\
\hline $\mathrm{t}_{1}$ & \multicolumn{2}{|l|}{ Rotatable polarizer receives to $\left|\beta_{00}\right\rangle$} \\
\hline $\mathrm{t}_{2}$ & \multicolumn{2}{|c|}{$\begin{array}{l}\text { The computer emits a bit }\left(\text { in }_{2, \mathrm{c}} \text { ) and this result enters the C2Q interface. The output of the }\right. \\
\mathrm{C} 2 \mathrm{Q} \text { interface enters the first } \mathrm{X} \text { Pauli gate (in } \mathrm{t}_{2, \mathrm{q}} \text { ) according to the following equivalence: } \\
\{0,1\} \equiv\{|0\rangle,|1\rangle\} \text {, so that: }\end{array}$} \\
\hline & $0 \equiv|0\rangle$ & $1 \equiv|1\rangle$ \\
\hline $\mathrm{t}_{3}$ & $\begin{array}{l}X|0\rangle=|1\rangle, \text { then, } \\
\mathrm{P}_{\mathrm{H}} \text { is activated, therefore, }\left|\beta_{00}\right\rangle \text { collapses to } \\
|H\rangle \equiv|0\rangle \text {, i.e., entanglement disappears. }\end{array}$ & $\begin{array}{l}X|1\rangle=|0\rangle \text {, then, } \\
\mathrm{P}_{\mathrm{H}} \text { is not activated }\left(\mathrm{P}_{\mathrm{H}}=\mathrm{I} \text {, identity matrix), }\right. \\
\text { therefore, }\left|\beta_{00}\right\rangle \text { continues to advance, i.e., } \\
\text { entanglement is still active. }\end{array}$ \\
\hline $\mathrm{t}_{4}$ & $\begin{array}{l}X|1\rangle=|0\rangle \text {, then, } \\
\mathrm{P}_{\mathrm{V}} \text { is not activated }\left(\mathrm{P}_{\mathrm{V}}=\mathrm{I} \text {, identity matrix }\right) \text {, } \\
\text { therefore, }|H\rangle \equiv|0\rangle \text { continues to advance } \\
\text { until the exit. }\end{array}$ & $\begin{array}{l}X|0\rangle=|1\rangle, \text { then, } \\
\mathrm{P}_{\mathrm{V}} \text { is activated, therefore, }\left|\beta_{00}\right\rangle \text { collapses to } \\
|V\rangle \equiv|1\rangle \text {, i.e., entanglement disappears. }\end{array}$ \\
\hline $\mathrm{t}_{5}$ & $|H\rangle \equiv|0\rangle \equiv 0$ & $|V\rangle \equiv|1\rangle \equiv 1$ \\
\hline
\end{tabular}


As we can see, the results of Tables 1 and 2 agree completely, although there are two things that change in relation to those tables:

I. in Table 2, the entity that generates the results is a computer which gives the rotatable polarizer a bitstream where each bit is part of a process in which a man may have intervened or may intervene, which implies a control per se of the results, instead

II. in Table 1, all quantum measurement represented mainly by the intervention of a PBS results from an average of results, which for the case of the perfect coin of Figure 6, and a large number of attempts (or shots) results in the probabilities that can be observed to the right of said figure, that is, $50 \%$ for each of the two possible spins. But the case of Figure 10 is absolutely deterministic, i.e., no matter how many times $\left|\beta_{00}\right\rangle$ goes through the rotatable polarizer, if the bit that comes out of the computer is always the same, the outcomes will be too and that is perfectly controllable with the computer, in fact, the intervention of both PBS at the end of each beam of Figure 9 (i.e., after rotatable polarizer) is done on an already collapsed wave-function of the Bell state $\left|\beta_{00}\right\rangle$, that is, on a CBS $\{|0\rangle,|1\rangle\}$. Therefore, the whole experiment is equivalent to let $\left|\beta_{00}\right\rangle$ pass through the rotatable polarizer only once, which is in fact what will happen in any communication protocol based on this experiment.

In other words, human intervention in the results could be interpreted as a loaded coin. For example, a message could exclusively consist of a 1 (meaning "attack"), or a 0 (meaning "retreat"), with no more transmission than that, and with a 50\% occurrence versus nothing as a counterpart, which will be developed below. The implications of the configuration of Figure 10 , as well as the above preliminary conclusions on quantum communications are dramatic. 
Figure 11 represents the circuit implementation of the computer-controlled rotatable polarizer inside the configuration of Figure 9. Each outgoing bit of the computer will be present as outcomes, in both Alice's and Bob's hands.

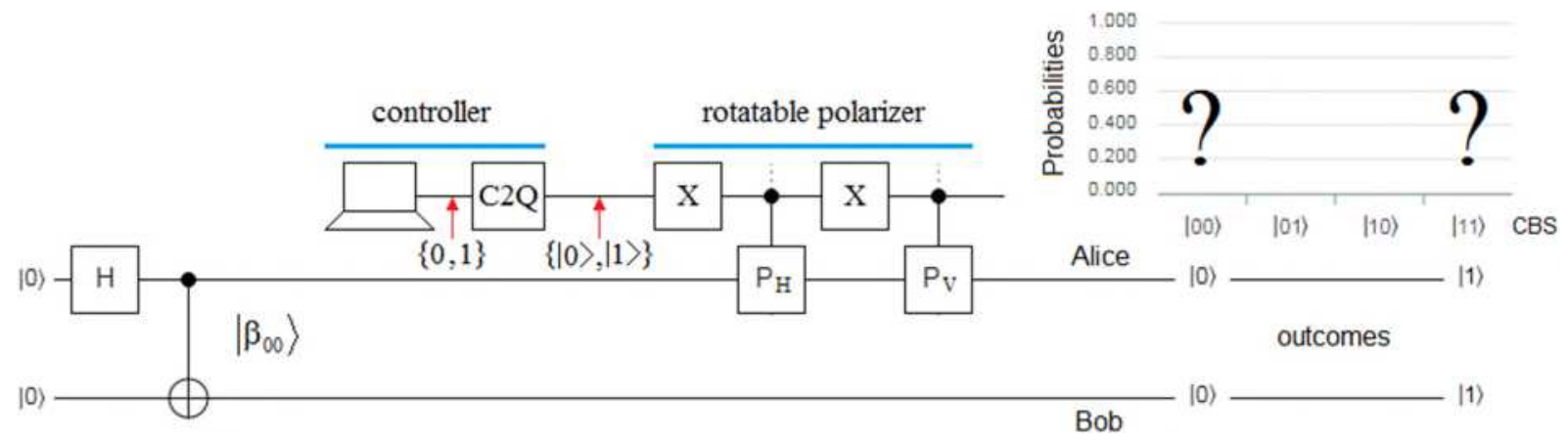

Figure 11. Circuit representation of Figure 9 configuration thanks to the rotatable polarizer modeled in Figure 10.

The reason why the probabilities on the right side of Figure 11 are missing has to do with what was mentioned before, which is equivalent to say that they depend on a voluntary process (deterministic) that takes place as each bit is emitted by the computer, which is generally unknown. However, taking into account the conclusions of Table 2, when the bit that comes out of the computer is $0 \equiv|0\rangle, \mathrm{P}_{\mathrm{H}}$ is activated, and $\mathrm{P}_{\mathrm{V}}$ is deactivated, which takes us to a simplified configuration of Figure 11 like that of Figure 12.

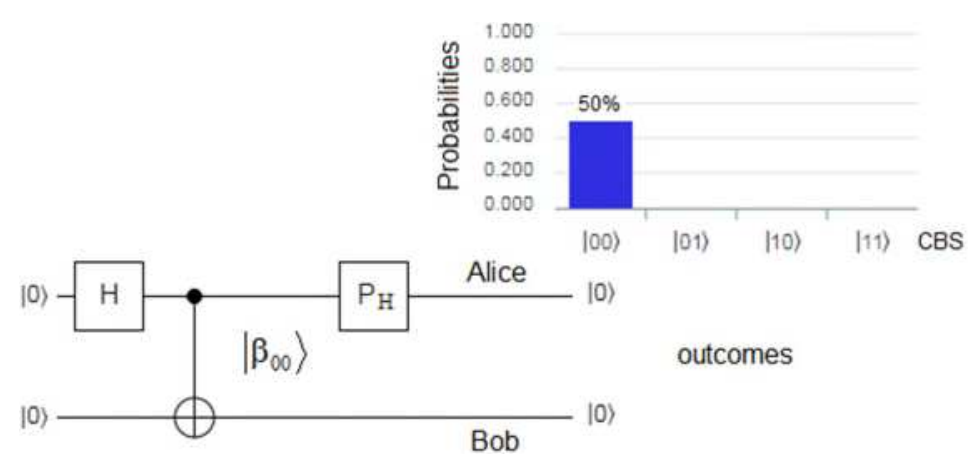

Figure 12. If the bit that comes out of the computer is $0 \equiv|0\rangle$, then, $P_{H}$ is only activated.

The result of applying the $\mathrm{P}_{\mathrm{H}}$ in Alice's beam can be calculated in the same way as we did for 
Equations 40 and 41 for the case of Figure 7, which is identical to Figure 12.

Now, when the bit that comes out of the computer is $1 \equiv|1\rangle, \mathrm{P}_{\mathrm{H}}$ is deactivated, and $\mathrm{P}_{\mathrm{V}}$ is activated, which refers us to a simplified configuration like that of Figure 13.

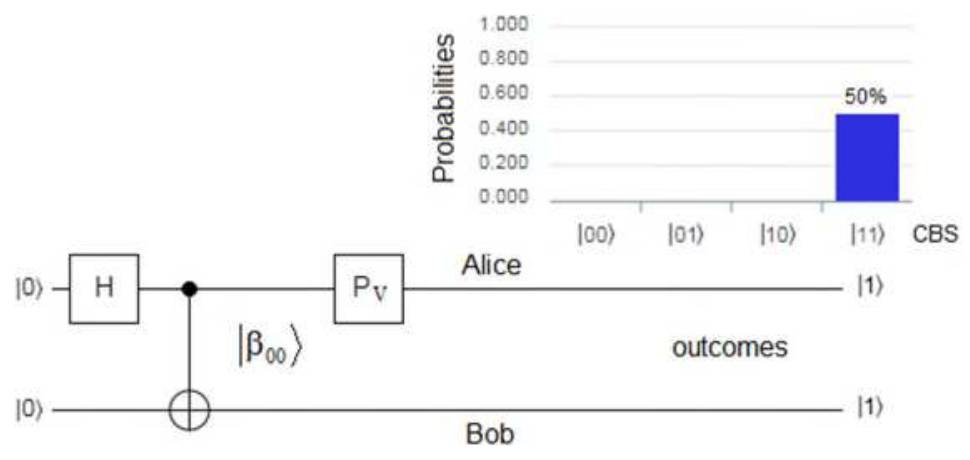

Figure 13. If the bit that comes out of the computer is $1 \equiv|1\rangle$, then, $P_{V}$ is only activated.

The result of applying the $P_{V}$ in Alice's beam can be calculated in the same way as we did for Equations 42 and 43 for the case of Figure 8, which is identical to Figure 13.

In other words, we have obtained the same results that nature obtained in Section 3 when we used a PBS to measure a Bell state $\left|\beta_{00}\right\rangle$ on an $\{|H\rangle,|V\rangle\}$ basis, without using a classical channel for instantaneous synchronization (post-distribution of the EPR pair) of the outcomes by using a suitable modeling of a controllable rotatable-polarizer restricted to only two possible polarizations: horizontal, and vertical. So the only difference between the configurations of Figures 6 and 11 is that in the latter we have full control over the outcomes of Alice and Bob. On the other hand, the transmission times involved in Figure 11 are the same as those for the case of Equation 44 of Section 3 for Figure 6, reaffirming the existence of an instantaneous synchronization posterior to the distribution of the EPR pair but with control over the outcomes. Moreover, if we see the configuration of Figure 11 as a communication protocol, this will be far from an instantaneous communication system, because of the time used in the distribution of the entangled pair, which monopolizes the total transmission time of the Equation 44, and which is clearly different from zero. 
Finally, the most important conclusion about the experiment of this section has to do with the possibility to control locally nonlocal outcomes on Bob's side, without using a classical channel for any type of synchronization and based on an appropriate configuration on Alice's side.

\section{Third experiment: quantum teleportation}

\subsection{Preliminaries}

In Figures 7 and 8 of Section 3 (Equations 40-43), we were able to see the outcomes obtained as well as the probabilities associated with the cases in which Alice's beam is interrupted with a horizontal $\left(\mathrm{P}_{\mathrm{H}}\right)$ or vertical $\left(\mathrm{P}_{\mathrm{V}}\right)$ polarizer, respectively. In both examples, it is made explicit that we will only have $50 \%$ of the total probabilities involved in each experiment. This has to do with the fact that the application of the $\mathrm{P}_{\mathrm{H}}$ polarizer only lets half of the components of $\left|\beta_{00}\right\rangle$ pass through it, i.e., $|00\rangle$, blocking the passage of $|11\rangle$, while the $\mathrm{P}_{\mathrm{v}}$ polarizer will do the opposite when it intervenes in Alice's beam. In order to verify these results on a platform available on the Internet, which allows us to define and use non-unitary matrices such as $\mathrm{P}_{\mathrm{H}}$ and $\mathrm{P}_{\mathrm{V}}$, we resorted to Quirk simulator ${ }^{[46]}$, in which we implemented both experiments again, and whose results can be seen in red in Figure 14(a, and b, respectively).

a

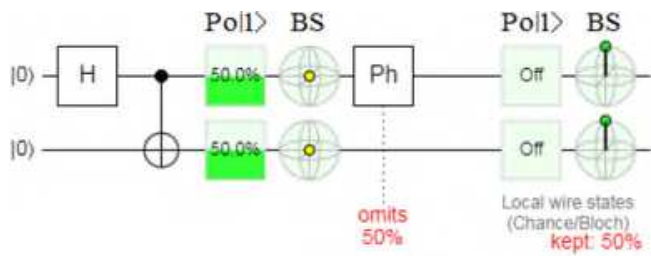

c

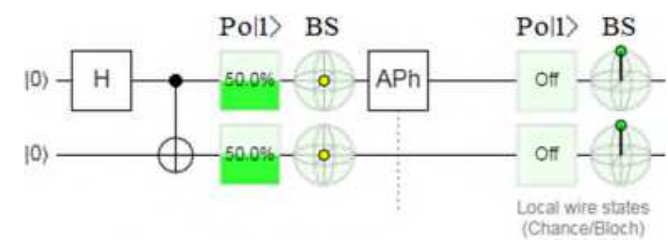

b

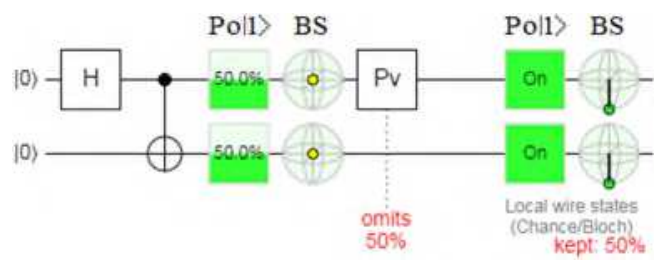

$\mathrm{d}$

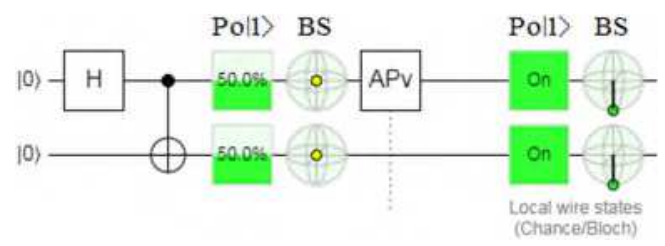

Figure 14. Implementation of the experiments of Figures 7 and 8 on Quirk simulator ${ }^{[46]}$, without (a, and b) and with (c, and d) amplification factor. 
Figure 14 shows two metrics, at the output of all circuit pairs $\{$ Hadamard gate $(\mathrm{H}), \mathrm{CNOT}\}$, where Po|1> means Probability of $\mid 1>$ (Equation 21 of Section 2), while BS represents Bloch's sphere. In all cases, Po| $1>$ is equal to $50 \%$, and we find a single yellow dot in the center of the Bloch sphere, which implies a typical representation of any of the Bell state of Equation 28, for example $\left|\beta_{00}\right\rangle$. The fundamental difference between the four graphs of Figure 14 is the percentage of retention of results indicated in red in graphs (a) and (b), which is not the case in graphs (c) and (d). This is because in the case of these last two graphs we use an amplification factor equal to $\sqrt{2}$. Wootters and Zurek ${ }^{[18]}$ explicitly commented on this issue: linearity does not forbid the amplification of any given state by a device designed especially for that state, but it does rule out the existence of a device capable of amplifying an arbitrary state. Therefore, in the cases of Figure 14 (c, and d), it is possible to apply such an amplification factor because a device should not be designed for a generic qubit but, especially, for CBS always, since in both cases (c) and (d), the application of the aforementioned amplification factor occurs after the intervention of the corresponding polarizer, that is, after $\left|\beta_{00}\right\rangle$ collapses into $|00\rangle$ or $|11\rangle$. As a result, maximum performance is reached in the outcomes obtained. This information is of vital importance for the different implementations that will be carried out in the next two subsections.

\subsection{Quantum teleportation}

Figure 15 shows the insertion of the PBS model of Figure 5 in the quantum teleportation protocol $^{[15-17]}$ prior to the use of a classical disambiguation channel. 


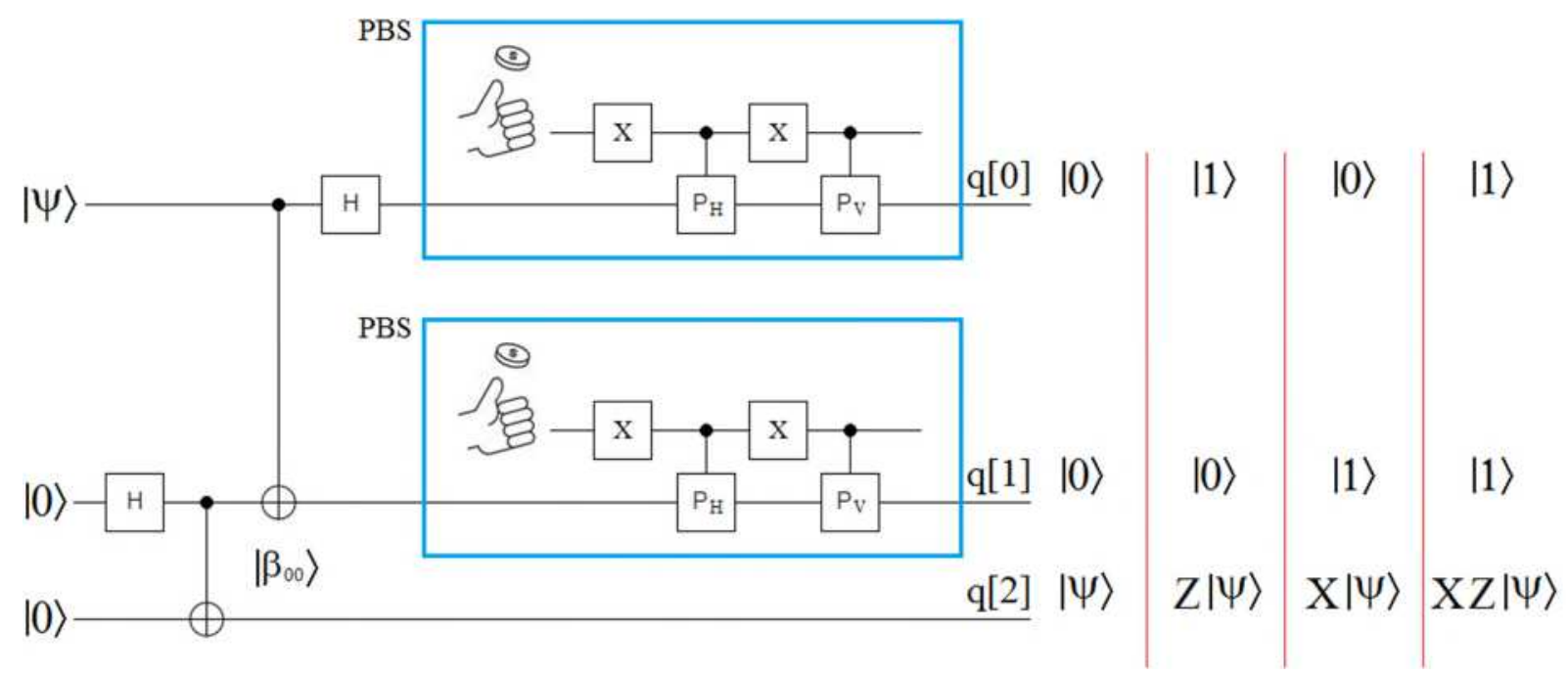

Figure 15. PBS model of Figure 5 inserted in the quantum teleportation protocol prior to the use of the classical disambiguation channel.

Four possible triads are randomly generated between both PBS:

$|q[0] q[1] q[2]\rangle \rightarrow\{|0\rangle|0\rangle|\psi\rangle,|0\rangle|1\rangle|X \psi\rangle,|1\rangle|0\rangle|Z \psi\rangle,|1\rangle|1\rangle|X Z \psi\rangle\}$.

For every combination of $\mathrm{q}[0] \mathrm{q}[1]$, we will have a certain state in $\mathrm{q}[2]$, which indicates that the protocol of Figure 15 cannot, per se, establish the correct teleportation of the qubit $|\psi\rangle$ in this way, since Bob has no way of knowing which of the four options is correct. For this reason, it is required to establish the correct state by transmitting the result of both PBS to Bob in order to use said results as a control or disambiguation bits and, thus, set the final value of the teleported qubit through the appropriate activation of a unitary-transform of reconstruction.

Therefore, we resort to the full teleportation protocol ${ }^{[15-17]}$ of Figure 16, which transmits both disambiguation bits $q[0] q[1] \equiv b_{Z} b_{X}$ using a classical channel (red) by activating the appropriate unitary-transform in $q[2]$ in order to reconstruct the teleported state $|\psi\rangle$. 


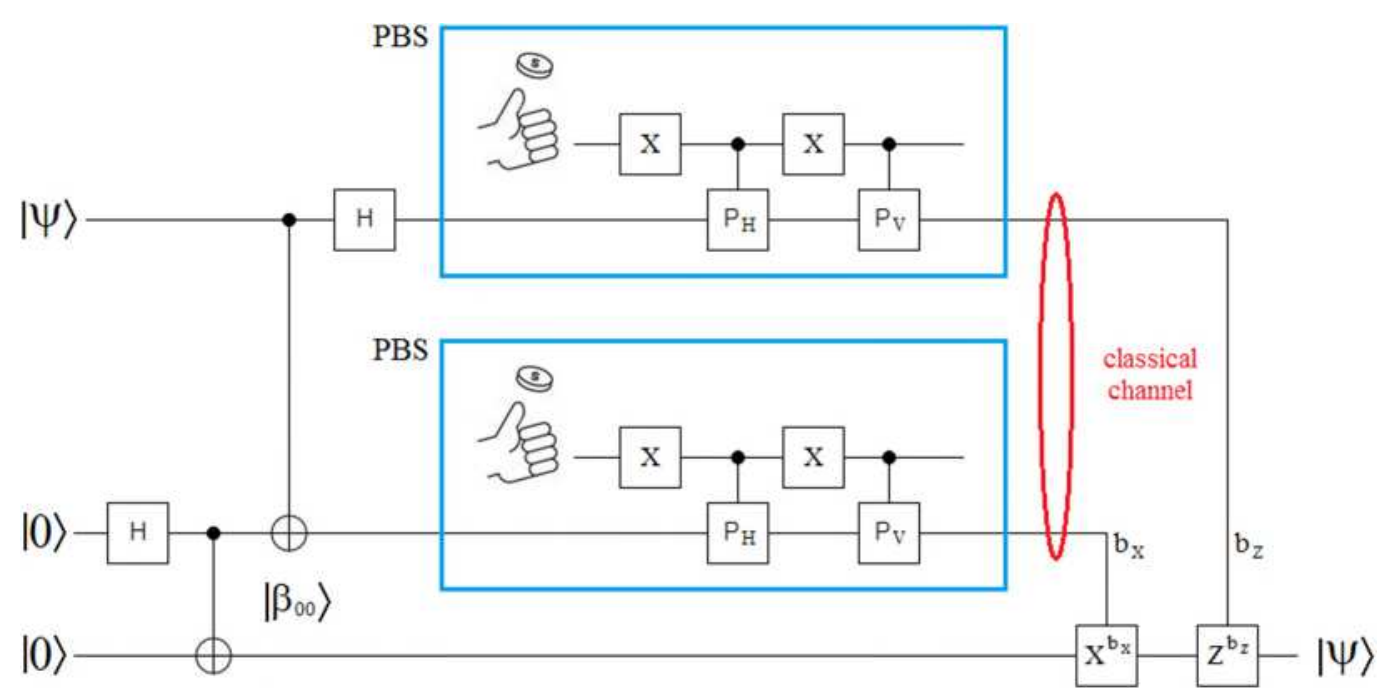

Figure 16. Complete teleportation protocol with the PBS model of Figure 5, both bits $\left\{b_{X}, b_{Z}\right\}$ of disambiguation, a classical channel, and a unitary-transform composed of $Z^{b_{Z}} X^{b_{X}}$.

As an example, let us use the protocol of Figure 16 to teleport the qubit that will be defined next. This example will allow us to numerically evaluate the operation of this protocol.

$$
\begin{aligned}
& \left|\psi_{1}\right\rangle=H T H T|0\rangle=\frac{1}{\sqrt{2}}\left[\begin{array}{cc}
1 & 1 \\
1 & -1
\end{array}\right]\left[\begin{array}{cc}
1 & 0 \\
0 & e^{i \frac{\pi}{4}}
\end{array}\right] \frac{1}{\sqrt{2}}\left[\begin{array}{cc}
1 & 1 \\
1 & -1
\end{array}\right]\left[\begin{array}{cc}
1 & 0 \\
0 & e^{i \frac{\pi}{4}}
\end{array}\right]\left[\begin{array}{l}
1 \\
0
\end{array}\right]=\left[\begin{array}{l}
0.85355+i 0.35355 \\
0.35355-i 0.14645
\end{array}\right], \\
& \text { Probabilities }_{\left|\psi_{1}\right\rangle}=\left[\begin{array}{l}
P o|0\rangle_{\left|\psi_{1}\right\rangle} \\
P o|1\rangle_{\left|\psi_{1}\right\rangle}
\end{array}\right]=\left[\begin{array}{l}
85.3553 \% \\
14.6447 \%
\end{array}\right] .
\end{aligned}
$$

Table 3 allows us to observe the qubit prepared by Alice in the metrics of Quirk simulator ${ }^{[46]}$, as well as the four options resulting from its measurement. Based on these four options, Bob instantly receives one of the four options in the first row corresponding to Bob in Table 3 after Alice's measurement. Bob corrects according to the two disambiguation bits $\left(b_{x}, b_{z}\right)$ sent by Alice through the classical channel, as we can see in the second row corresponding to Bob in Table 3. Those bits activate the appropriate gates of the unitary-operator on Bob's side in order to reconstruct the teleported state $|\psi\rangle$.

Table 3. Alice prepares qubit to be teleported, measures $\mathrm{q}[0]$ and $\mathrm{q}[1]$, and obtains disambiguation 
bits $\left(b_{x}, b_{z}\right)$. Bob receives $\left(b_{x}, b_{z}\right)$, corrects $q[2]$ with the corresponding unitary-operator, and gets the final outcome of $|\psi\rangle$.

\begin{tabular}{|c|c|c|c|c|c|}
\hline \multirow{2}{*}{ Alice } & $\begin{array}{c}\text { prepares } \\
|\psi\rangle\end{array}$ & \multicolumn{4}{|c|}{ Pol1> BS } \\
\hline & $\begin{array}{c}\text { measures } \mathrm{q}[0] \\
\text { and } \mathrm{q}[1], \text { and } \\
\text { obtains }\left(\mathrm{b}_{\mathrm{X}}, \mathrm{b}_{\mathrm{Z}}\right)\end{array}$ & $(|0\rangle,|0\rangle)$ & $(|0\rangle,|1\rangle)$ & $(|1\rangle,|0\rangle)$ & $(|1\rangle,|1\rangle)$ \\
\hline \multirow{3}{*}{ Bob } & $\begin{array}{c}\text { receives } \\
\left(b_{x}, b_{z}\right)\end{array}$ & 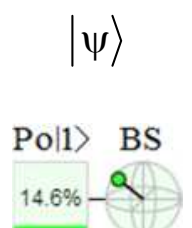 & $\begin{array}{r}X|\psi\rangle \\
\text { Po|1> BS } \\
14.6 \%->\end{array}$ & $\begin{array}{r}Z|\psi\rangle \\
\text { Po|1〉 BS } \\
85.4 \%-\end{array}$ & $\begin{array}{c}X Z|\psi\rangle \\
\text { Po|1> BS } \\
85.4 \%-\frac{d}{6}\end{array}$ \\
\hline & $\begin{array}{l}\text { corrects } \mathrm{q}[2] \\
\text { with the } \\
\text { corresponding } \\
\text { unitary-operator }\end{array}$ & $|\psi\rangle$ & $X(X|\psi\rangle)$ & $Z(Z|\psi\rangle)$ & $Z X(X Z|\psi\rangle)$ \\
\hline & $\begin{array}{l}\text { gets } \\
|\psi\rangle\end{array}$ & 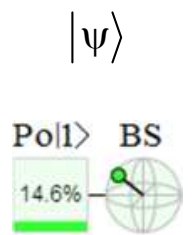 & 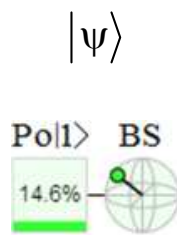 & $\begin{array}{l}\quad|\psi\rangle \\
\text { Po|1 } \mathrm{BS} \\
14.6 \%\end{array}$ & $\begin{array}{l}\quad|\psi\rangle \\
\text { Po|1> BS } \\
14.6 \% \_9\end{array}$ \\
\hline
\end{tabular}

Figure 17 presents the insertion of the rotatable polarizer model of Figure 10 in the quantum teleportation protocol ${ }^{[15-17]}$ prior to the use of a classical disambiguation channel.

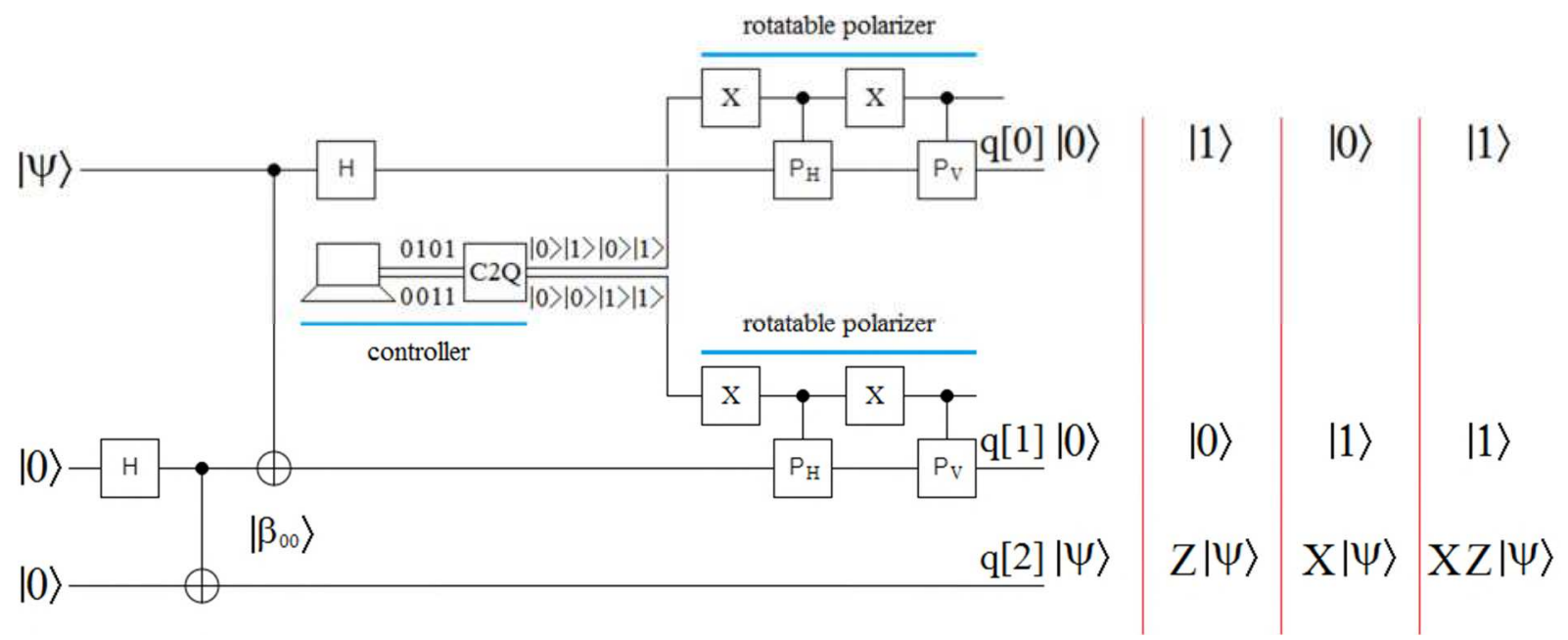

Figure 17. Rotatable polarizer model of Figure 10 inserted in the quantum teleportation protocol prior to the use of a classical disambiguation channel. 
Unlike the case of Figure 11 of Section 4, here, the controller (computer + C2Q interface) controls two rotatable polarizers at the same time: in the upper wire it sends a sequence of bits 0101, while in the lower wire it sends a sequence of bits 0011. In this way, the controller covers the four options necessary for the analysis of Figure 17. The idea behind this figure is to emulate the four possible options of Figure 15 but in a controlled way, i.e., at will. This last point is extremely important, since if we analyze the four options to the right of Figure 17 we can see that when the controller generates zeros in its two outputs, then $q[0]=|0\rangle$ and $q[1]=|0\rangle$, then Bob directly receives $|\psi\rangle$. Since we can at will control which option there will always be at the output of both rotatable polarizers, we always select $q[0]=|0\rangle$ and $q[1]=|0\rangle$, in which case Bob always receives the correct option, i.e. directly the teleported state $|\psi\rangle$. All of this happens without using a classical disambiguation channel, control (or disambiguation) bits, or a unitary transform on Bob's side. This is clearly seen in Table 3, but it happens that said table is associated with the modeling of the PBS, in which case, we do not have external control of what happens with the internal process that takes place in each of them. In fact, if we set both controller outputs to 00 , this is equivalent to exclusively activating the $\mathrm{P}_{\mathrm{H}}$ horizontal polarizers on both rotatable polarizers at the same time, which in turn is equivalent to the protocol of Figure 18. In this figure, the only measurement process that takes place is that on the teleported qubit $|\psi\rangle$ on Bob's side, since both horizontal polarizers $\mathrm{P}_{\mathrm{H}}$ act deterministically on their respective qubits like any quantum gate.

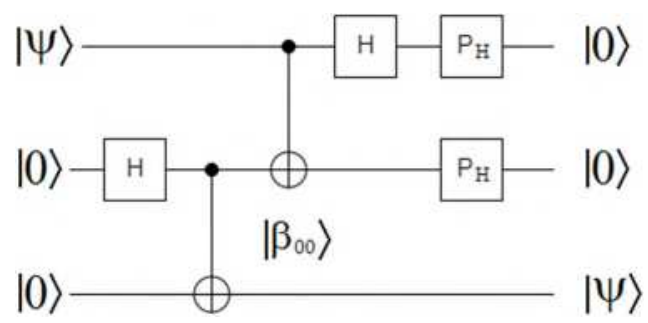

Figure 18. Equivalent circuit of the protocol of Figure 17 when both controller outputs are 
simultaneously 00 , which exclusively activates the $\mathrm{P}_{\mathrm{H}}$ on both rotatable polarizers.

Next, we will use the protocol of Figure 18 for the teleportation of the qubit of Equations 46 and 47, thanks to an implementation on Quirk simulator ${ }^{[46]}$, which can be seen in Figure 19. In this figure, we must pay particular attention to the inscriptions in red below the $\mathrm{P}_{\mathrm{H}}$ and the final metrics, given that the $\mathrm{P}_{\mathrm{H}}$ located in $\mathrm{q}[0]$ eliminates $50 \%$ of the information that crosses it, while the $\mathrm{P}_{\mathrm{H}}$ located in $\mathrm{q}[1]$ eliminates $50 \%$ of $50 \%$ of the information that passes through it, that is, $25 \%$. Therefore, both $\mathrm{P}_{\mathrm{H}}$ eliminate $75 \%$ of the information traffic that passes through both, which results in that Bob will only keep $25 \%$ of the outcomes in play in q[2]. Nonetheless, the teleportation is completely successful, which is evidenced by comparing the metrics at the end of qubit preparation in $\mathrm{q}[0]$ with those in $\mathrm{q}[2]$ to the right of Figure 19.

Another successful application of the protocol of Figure 18 can be seen in Figure $\mathbf{2 0}$ associated with the teleportation of the following qubit:

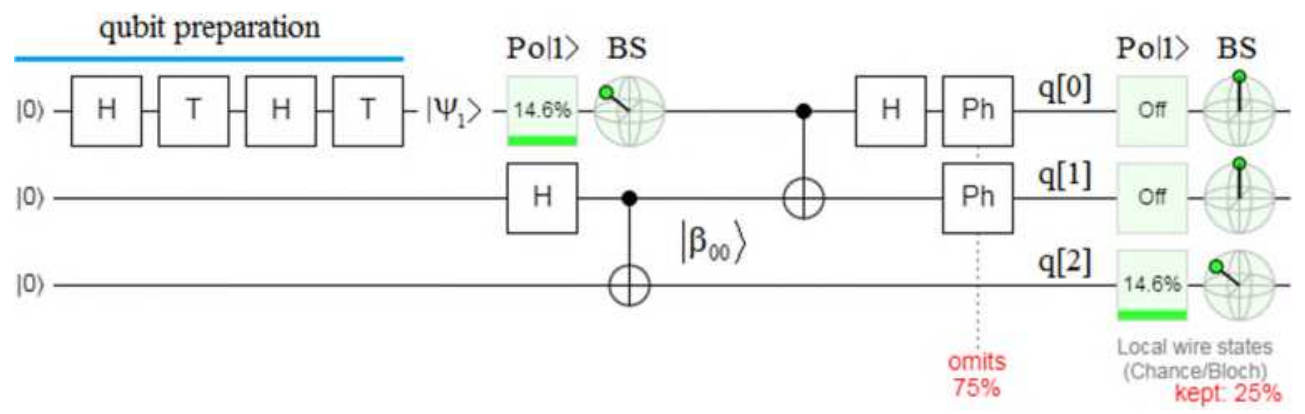

Figure 19. Teleportation of the qubit of Equations 46 and 47 using the protocol of Figure 18 on Quirk simulator. ${ }^{[46]}$

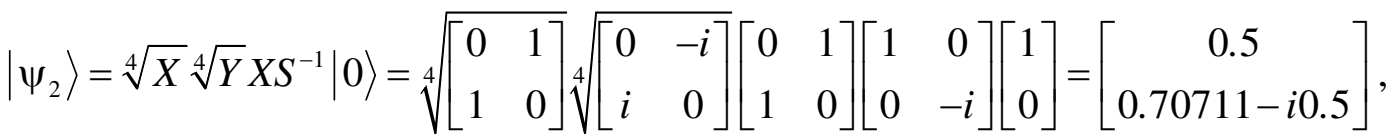

$$
\begin{aligned}
& \text { Probabilities }_{\left|\psi_{2}\right\rangle}=\left[\begin{array}{c}
P o|0\rangle_{\left|\psi_{2}\right\rangle} \\
P o|1\rangle_{\left|\psi_{2}\right\rangle}
\end{array}\right]=\left[\begin{array}{c}
25 \% \\
75 \%
\end{array}\right] \text {. }
\end{aligned}
$$

Figure 20 shows as the metrics at the end of qubit preparation in $\mathrm{q}[0]$ are identical to those in 
$\mathrm{q}[2]$ to the right of this figure, with the same degree of omission of information by both $\mathrm{P}_{\mathrm{H}}$ (75\%) and recovery of outcomes from Bob's side (25\%) as in Figure 19.

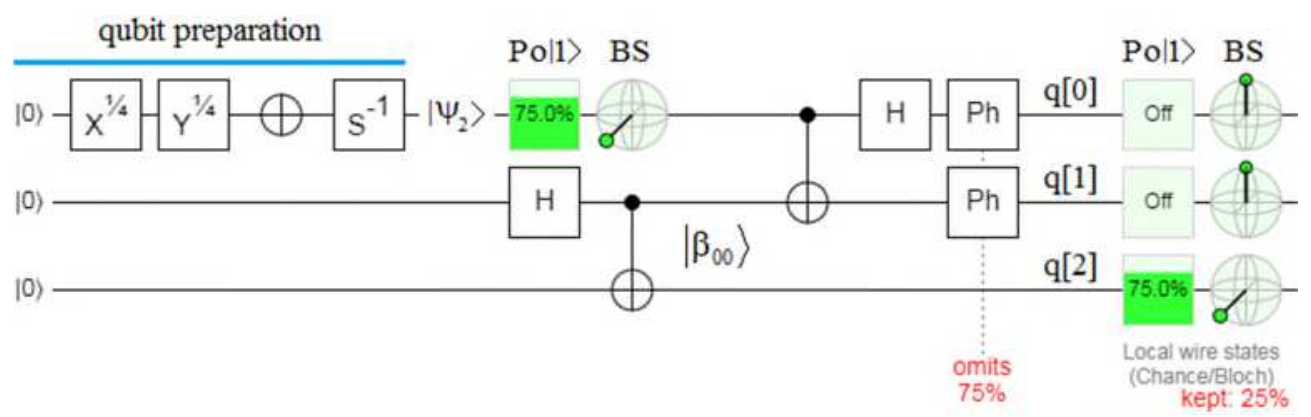

Figure 20. Teleportation of the qubit of Equations 48 and 49 using the protocol of Figure 18 on Quirk simulator. ${ }^{[46]}$

If we now apply the same amplification factor $\sqrt{2}$ of Figure 14 (c, and $d)$ to the output of both $\mathrm{P}_{\mathrm{H}}$ of Figures 19 and 20, we will obtain the configurations of Figures 21 and 22.

In both figures, the amplification factor present at the output of each horizontal polarizer is represented as $\mathrm{AP}_{\mathrm{H}}=\sqrt{2} \mathrm{P}_{\mathrm{H}}$. As we can see, the percentages about the information suppressed at the output of each $\mathrm{P}_{\mathrm{H}}$ due to the recovery by the aforementioned amplification factor are absent in both figures, which allows Bob to recover $100 \%$ of the outcomes at stake.

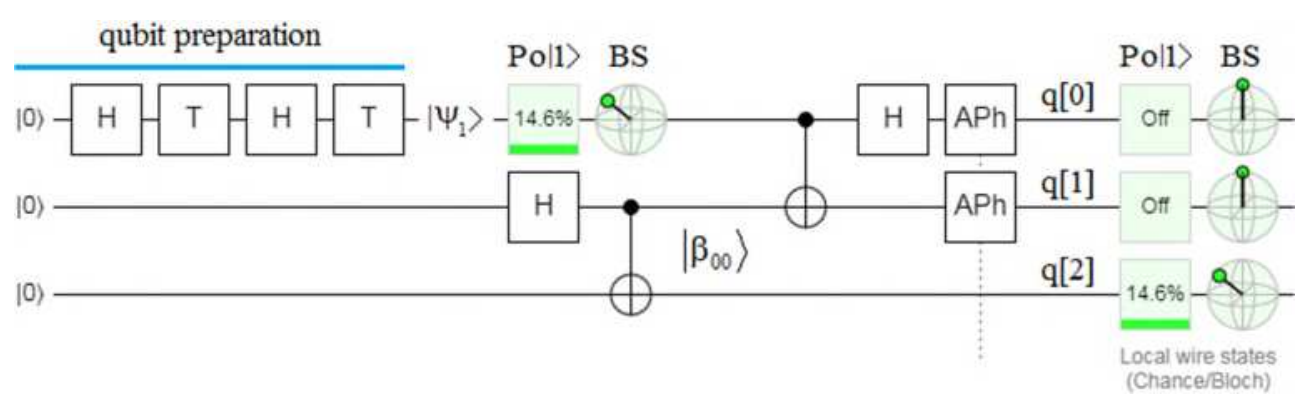

Figure 21. Teleportation of Figure 19 with amplification factor, where $A P h=\sqrt{2} \mathrm{P}_{\mathrm{H}}$. 


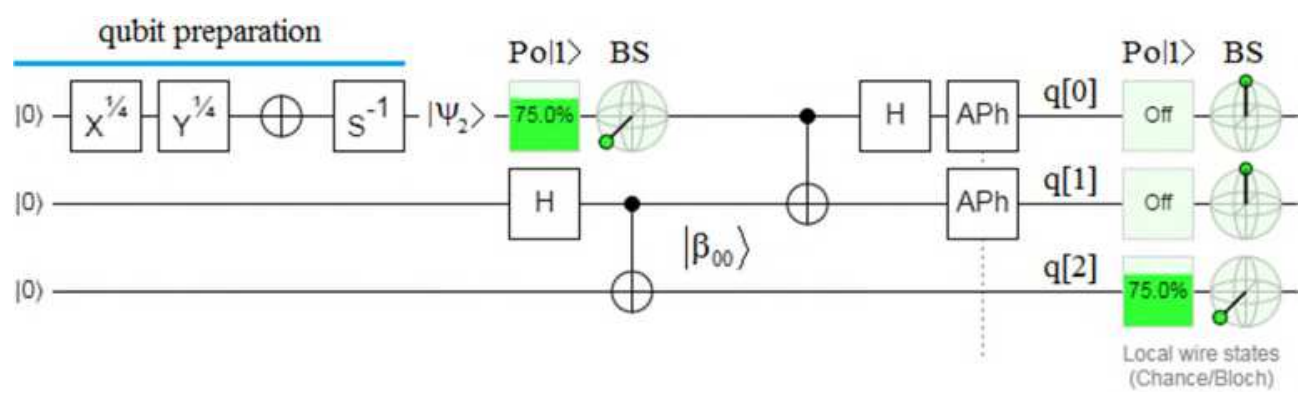

Figure 22. Teleportation of Figure 20 with amplification factor, where $A P h=\sqrt{2} \mathrm{P}_{\mathrm{H}}$.

\subsection{Projection on the quantum Internet}

As a natural extension of the application of the concepts seen in the previous subsection, we will use horizontal polarizers $\mathrm{P}_{\mathrm{H}}$ in the main protocols present in Quantum Internet ${ }^{[23-36]}$, that is, Quantum Secret Sharing (QSS) $)^{[47]}$, Entanglement Swapping (ES) ${ }^{[48]}$, and Quantum Repeaters (QR). ${ }^{[49-53]}$

\subsubsection{Quantum Secret Sharing}

The implementation of the quantum secret sharing protocol for the case of three entangled photons, where we work with a configuration of the $\mathrm{GHZ}_{3}$ (Daniel M. Greenberger, Michael

A. Horne, and Anton Zeilinger) type, is presented in Figure 23. $\mathrm{GHZ}_{3}$ state is expressed as:

$$
\left|G H Z_{3}\right\rangle=\frac{1}{\sqrt{2}}(|000\rangle+|111\rangle)
$$

For this experiment, we prepare a qubit identical to the one used for the case of Equations 46 and 47. Figure 23(a) shows the original version of this protocol using three PBS, a classical channel, and a unitary-transform on Bob's side, i.e., qubit q[3], in order to reconstruct the transferred state $|\psi\rangle$. Figure 23(b) presents a version of the QSS protocol replacing the three PBS, the classical channel, and the unitary-transform on Bob's side of the version (a) with three horizontal polarizers $\mathrm{P}_{\mathrm{H}}$ on qubits $\mathrm{q}[0]$, $\mathrm{q}[1]$, and $\mathrm{q}[2]$. In fact, $\mathrm{P}_{\mathrm{H}}$ on qubit $\mathrm{q}[0]$ omits $50 \%$ of information that goes through it, $\mathrm{P}_{\mathrm{H}}$ on qubit $\mathrm{q}[1]$ omits $50 \%$ of $50 \%$ of information that goes 
a

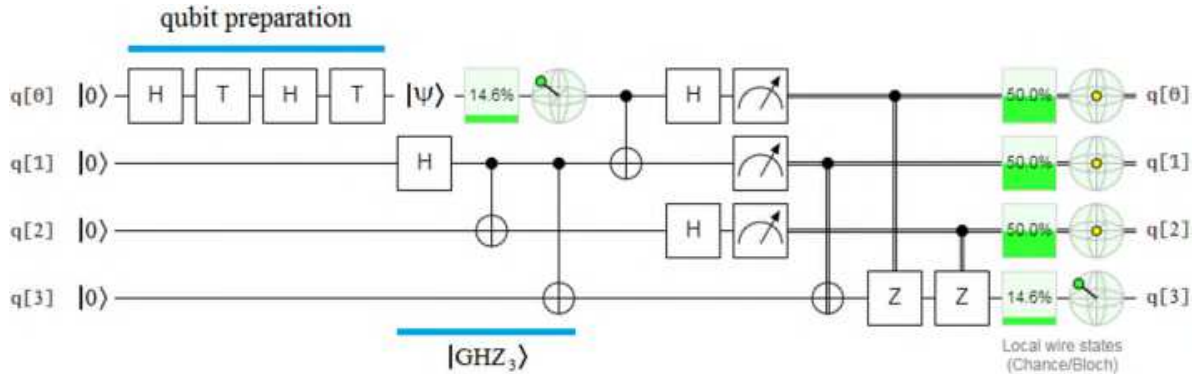

b

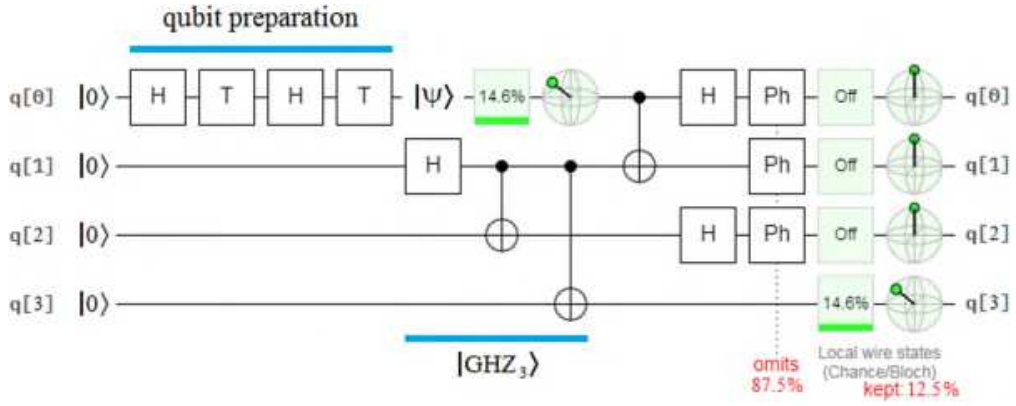

c

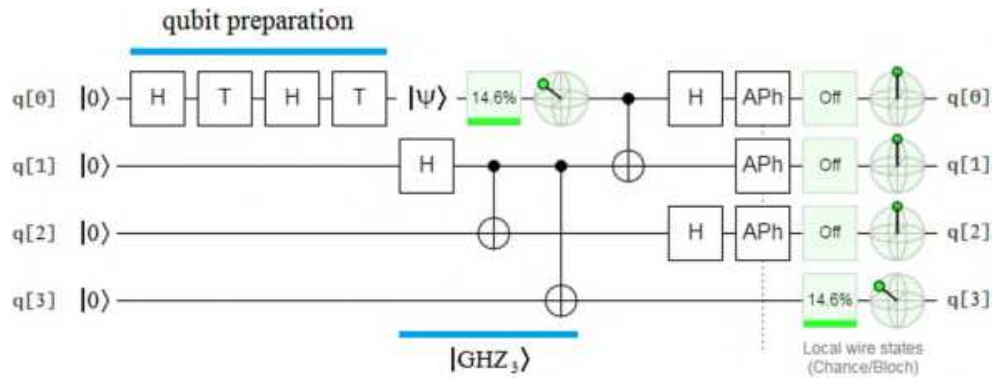

Figure 23. Quantum Secret Sharing protocol: a) original with a classical channel, b) version with horizontal polarizer $\mathrm{P}_{\mathrm{H}}$, and c) version with a horizontal polarizer, and an amplification factor, $\mathrm{AP}_{\mathrm{H}}$.

through it, i.e., $25 \%$, while $\mathrm{P}_{\mathrm{H}}$ on qubit q[2] omits $50 \%$ of $50 \%$ of $50 \%$ of information that goes through it, i.e., $12.5 \%$. Between the three $\mathrm{P}_{\mathrm{H}}, 87.5 \%$ of the information that passes through them is blocked. In consequence, Bob only recovers the $12.5 \%$ of the outcomes at stake. Finally, Figure 23(c) is similar to the previous one but incorporating the amplification factor at the output of every $\mathrm{P}_{\mathrm{H}}$, with which Bob recovers $100 \%$ of the outcomes at stake.

\subsubsection{Entanglement Swapping}

Figure 24 shows three versions of the entanglement swapping protocol. The first version, Figure 24(a) represents the original protocol with two PBS, two classical bits, and two classical channels, one from the middle up (from q[1] to q[0]), and one from the middle down (from q[2] 
a

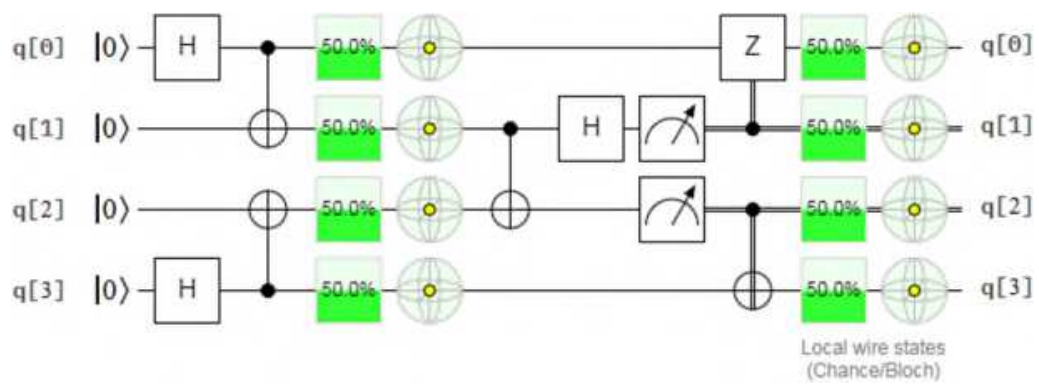

b

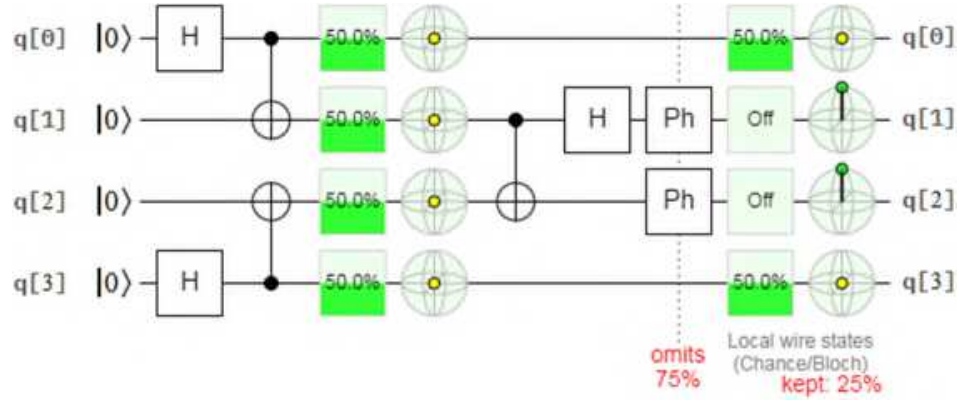

c

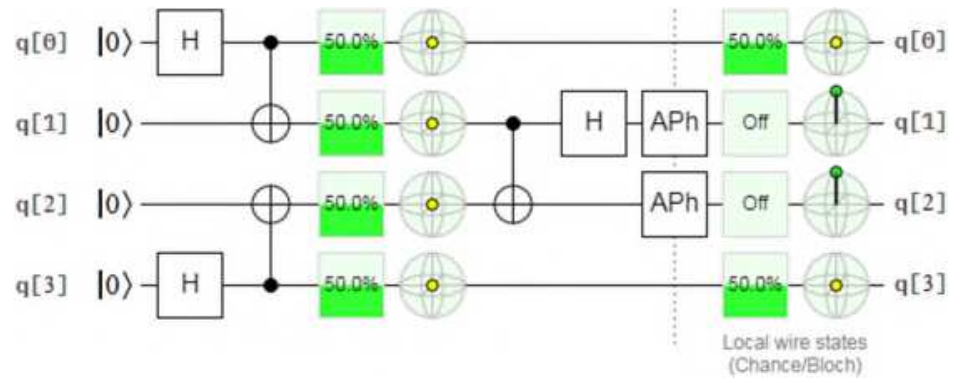

Figure 24. Entanglement Swapping protocol: a) original with a classical channel, b) version with horizontal polarizer $\mathrm{P}_{\mathrm{H}}$, and c) version with a horizontal polarizer, and an amplification factor, $\mathrm{AP}_{\mathrm{H}}$.

to $\mathrm{q}[3]$ ). Figure 24(b) shows a version based on the use of horizontal polarizers $\mathrm{P}_{\mathrm{H}}$, where the first $\mathrm{P}_{\mathrm{H}}$ (on $\mathrm{q}[1]$ ) retains $50 \%$ of the information that passes through it, while the second $\mathrm{P}_{\mathrm{H}}$ (on q[2]) retains $50 \%$ of $50 \%$. Together, both $\mathrm{P}_{\mathrm{H}}$ retain $75 \%$ and thus the entanglement between qubits $\mathrm{q}[0]$ and $\mathrm{q}[3]$ (on the right side of this figure) recovers $25 \%$ of the outcomes at stake. Finally, Figure 24(c) presents an identical configuration to that of Figure 24(b) but with an amplification factor of $\sqrt{2}$ after every horizontal polarizer $\mathrm{P}_{\mathrm{H}}$. As a direct consequence of this, entanglement between qubits $\mathrm{q}[0]$ and $\mathrm{q}[3]$ (on the right side of this figure) recovers $100 \%$ of the outcomes at stake.

\subsubsection{Quantum Repeaters}


Quantum repeater is a key piece in the world of quantum Internet when it is necessary to transmit information to long distances, both in the case of transmissions using optical fiber lines ${ }^{[49,51-53]}$, and in the case of using satellite relays. ${ }^{[50,54-57]}$ In fact, the first one have the following disadvantages: a propagation speed $(v)$ equal to $2 / 3$ of the speed of light $(c)$, losses and an attenuation in the material that requires the installation of a repeater every $50 \mathrm{~km}$, while satellite repeaters can cover greater distances at a speed $v=c$, with less attenuation and losses than in the case of optical fiber except for relative environmental aspects to the ground-sky link, i.e., clouds that can disrupt the distribution of entangled photons. ${ }^{[55-57]}$ Whether a satellite or terrestrial version is used, the quantum repeater protocol is based on the entanglement swapping protocol of Figure 24.

Figure 25(a) represents the original version of the quantum repeater protocol, where, we can see the qubit preparation module on $\mathrm{q}[0]$, while from $\mathrm{q}[1]$ to $\mathrm{q}[4]$ the entanglement swapping protocol is embedded. This version features four PBS, three classical channels, and one unitary transform on $\mathrm{q}[4]$ (on the right side of this figure) so that Bob can reconstruct the transmitted qubit. Figure 25(b) shows a version of quantum repeater protocol based on four horizontal polarizers $\mathrm{P}_{\mathrm{H}}$, two in the entanglement swapping module, and two more on the $\mathrm{q}[0]$ and $\mathrm{q}[1]$ qubits (on the right side of this figure) for the teleportation procedure of the qubit $|\psi\rangle$. In fact, the first $\mathrm{P}_{\mathrm{H}}$ (on q[2]) omits $50 \%$ of the information that passes through it, while the second $\mathrm{P}_{\mathrm{H}}$ (on q[3]) omits $50 \%$ of $50 \%$ of the information that crosses it. Together, they block $75 \%$ of the information involved in the protocol. The remaining $25 \%$ will be available to the $\mathrm{P}_{\mathrm{H}}$ of the qubits $\mathrm{q}[0]$ and $\mathrm{q}[1]$. The former will omit $50 \%$ of that $25 \%$, while the latter will omit $50 \%$ of $50 \%$ of $25 \%$. Together they will omit $18.25 \%$ which added to the $75 \%$ of the first two $\mathrm{P}_{\mathrm{H}}$ makes a total of $93.75 \%$ omitted by the four $\mathrm{P}_{\mathrm{H}}$. Then, Bob only recovers a $6.25 \%$ of the outcomes at stake. Finally, by replacing the four $\mathrm{P}_{\mathrm{H}}$ of Figure 25(b) with four $\mathrm{P}_{\mathrm{H}}$ with amplification factor of $\sqrt{2}$, Bob recovers $100 \%$ of the outcomes in Figure 25(c). 
a

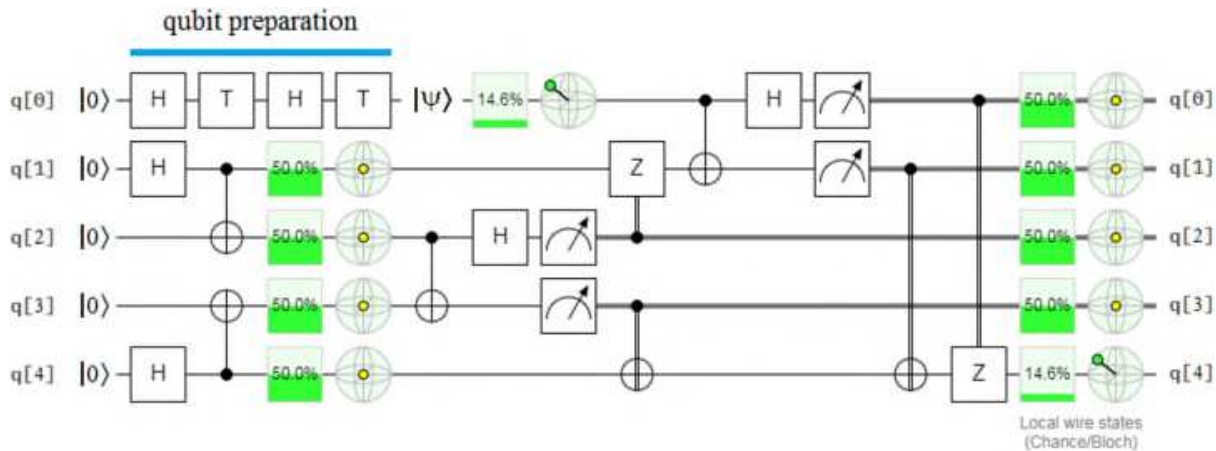

b

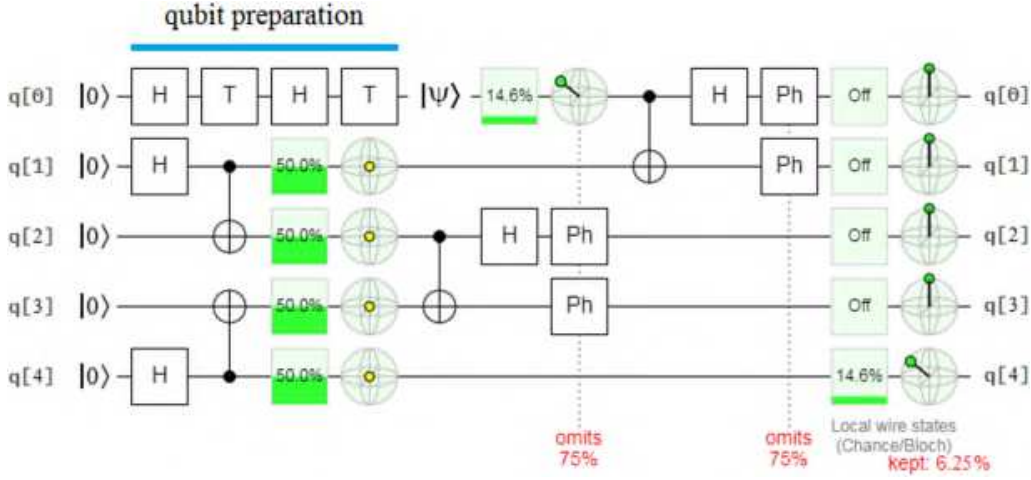

c

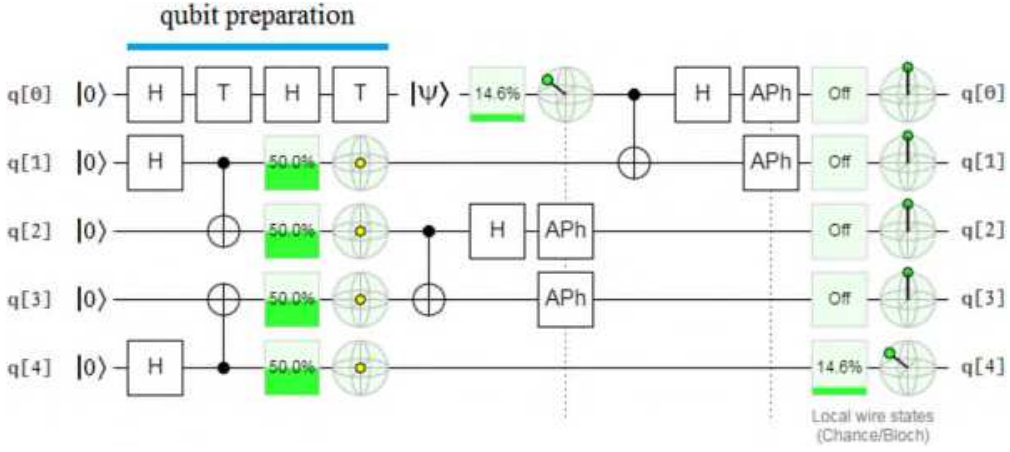

Figure 25. Quantum Repeater protocol: a) original with a classical channel, b) version with horizontal polarizer $\mathrm{P}_{\mathrm{H}}$, and c) version with a horizontal polarizer, and an amplification factor, $\mathrm{AP}_{\mathrm{H}}$.

\section{Interpretation of the results, and direct consequences of the model}

In this section, we will analyze the consequences of the two models presented before, that is, that of the PBS, and that of the rotatable polarizer, which led to the deterministic simplification of Figure 18. Consequently, this section is organized into three groups or subsections:

- interpretation of the results, mainly from the two immediately preceding sections,

- direct consequences of the models, in particular, in relation to the internal representation of the PBS, its contribution to a new approach about randomness and instantaneity in the synchronization of outcomes as a consequence of a quantum measurement, and if this really 
confronts two of the main pillars of Physics: Special Relativity and Quantum Mechanics; having as a central axis the possibility of locally controlling non-local outcomes, and

- time analysis, trying to establish definitively that an instantaneity of the process that takes place after the distribution of the entangled pairs; be it without control, with quasi-control, or with complete local control of non-local outcomes; does not imply at all the fact that the communication system that uses the entanglement is instantaneous, precisely because of the initial relativistic time for the distribution of the entangled pair, which gives rise to the essential quantum channel.

\subsection{Interpretation of the results}

The closed model of a PBS, presented in Figure 5, can be emulated with the open model of the rotatable polarizer of Figure 10, as long as the computer leading the control generates random numbers. In that case, both models will work statistically and functionally in the same way, to the point that the model in Figure 10 perfectly represents the model in Figure 5.

Another highlight of the two previous sections is that both with the PBS model used for the quasi-control of Figure 6, and for the case of the rotatable polarizer model used for the control of Figure 11, the instantaneous synchronization of the outcomes (not locals to each other) did not require a classical channel of control or disambiguation to carry it out. These successful results made it possible to extend the applicability of both models to the quantum teleportation protocols of Figures 15 to 17. First in the case of Figure 17 and then in the simplified model of Figure 18, we can see that both $\mathrm{P}_{\mathrm{H}}$ break the ambiguity present in the original protocols of Figures 15 and 16, since in Figure 17 the possibility of choosing the option 00 for the qubits $\mathrm{q}[0]$ and $\mathrm{q}[1]$ automatically converts the entire process into deterministic, which exempts the use of the traditional classical channel used as a sine qua non condition in the original quantum teleportation protocol. ${ }^{[15]}$ In other words, in Figure 17, something else other than the control of a computer is present: our will to use it, which is equivalent to our will being present in the result of the experiment. This criterion was extended with the same successful results to the use 
of $\mathrm{P}_{\mathrm{H}}$ in new versions of the protocols of quantum secret sharing, entanglement swapping, and quantum repeaters, contributing to a quantum Internet without classical channels.

\subsection{Direct consequences of the models}

This subsection revolves around the question of the Abstract, which has been present in Physics for the last hundred years: How a random phenomenon between particles can be synchronized instantaneously and independently of the distance between said particles? Several elements are entangled in this question: entanglement, quantum measurement, randomness, instantaneity, and space; giving rise to the aforementioned conflict between Special Relativity ${ }^{[4]}$ and Quantum Mechanics $^{[5]}$, regardless of whether or not the man can take advantage of the aforementioned instant synchronization. As a direct consequence of this question, others arise: Which actor are we talking about when we say random phenomenon: Entanglement, quantum measurement, or a conjunction between them? In fact, the previous one, is another way of asking: What is the responsible for randomness: Entanglement, quantum measurement, or both? What is the responsible for the instant synchronization of non-local results: Entanglement, quantum measurement, or both? Is there a synchronization of the entanglement prior to quantum measurement?

The result of a quantum measurement of entangled particles is random, no doubt about that, in fact, the quantum random number generators used today in quantum Cryptography ${ }^{[21,22]}$ and quantum Internet ${ }^{[23-36]}$ are based on this principle. However: Is it possible that constitutive instances of quantum measurement can be regular and yet quantum measurement as a whole be random?

The following question arises automatically: Does the PBS model of Section 4 help us in any way to clarify the previous questions? Moreover, would an internal model of the entanglement help us in any way to clarify the previous questions?

In Sections 4 and 5, we have established that the rotatable polarizer model is deterministic, since for the same inputs we will always have the same outputs. But, if it is a deterministic 
model, then: why in these sections do we talk about Probabilities of outcomes? Actually, we are talking about the probability that unpolarized light like $\left|\beta_{00}\right\rangle=1 / \sqrt{2}(|00\rangle+|11\rangle)$ will pass through a horizontal polarizer, which will always be 50\% (Figure 12, idem to Equation 41). We can also talk about the intensity of the light, before and after passing through the polarizer, in which case the intensity at the output is half that of the input.

As a fundamental strategy in order to answer the previous questions, we must separate the problem into parts, since trying to elucidate the origin of randomness with a strictly overall vision, as in fact happens with the literature on this subject, will not allow progress beyond that literature. To achieve this, we need to test the PBS model to the limit, create an internal model of the entanglement concomitant with this analysis, and evaluate in detail the human intervention in relation to the measurement process.

We are going to create an index of randomness of Boolean nature $\{$ Yes $\equiv 1$, No $\equiv 0\}$, which arises as an answer to a simple question about the randomness of the three actors in play: entanglement, PBS, and human intervention:

- $\quad$ Is entanglement random? Yes $=>\mathrm{RI}_{\mathrm{E}}=1, \mathrm{No}=>\mathrm{RI} \mathrm{I}_{\mathrm{E}}=0$,

- $\quad$ Is PBS random? Yes $=>$ RI $I_{P B S}=1$, No $=>R I_{P B S}=0$, and

- $\quad$ Is human intervention random? Yes $=>\mathrm{RI}_{\mathrm{HI}}=1$, No $\Rightarrow \mathrm{RI}_{\mathrm{HI}}=0$,

where, $\mathrm{RI}_{\mathrm{E}}, \mathrm{R} \mathrm{I}_{\mathrm{PBS}}, \mathrm{RI}_{\mathrm{HI}}$, are the randomness index of entanglement, PBS, and human intervention, respectively. With these indices we create a general index of post-measurement randomness:

$\mathrm{RI}_{\mathrm{PM}}=\mathrm{RI}_{\mathrm{E}} \vee \mathrm{RI} \mathrm{PBS}_{\mathrm{P}} \vee \mathrm{RI}_{\mathrm{HI}}$,

where, $\mathrm{RI}_{\mathrm{PM}}$ is the post-measurement random index, and “ $\mathrm{V}$ ” represents the logical OR operator. According to Equation 51, the index of post-measurement randomness RIPM will be 1 (random) with at least one of its constitutive indices equal to 1 . In other words, the quantum measurement process, as a whole, will be random as we know it with only one of the intervening factors being 
random, while the other two can be perfectly regular. Therefore, the strategy from here on will consist of forcing the regularity of each model to the maximum until reaching the one that can never be regular and with that scenario we will stay, thus automatically identifying the true factor responsible for the randomness resulting from all quantum measurement process. This hypothesis is based on the fact that a scenario where the three elements are random is much more complex and less consistent with the regularity and organization observed in the quantum world, after all the only randomness of the quantum world always revolves around the same: the direct or indirect estimation of the state of the variables involved, that is, their measurement. Therefore, applying the Ockham's razor ${ }^{[58,59]}$ : pluralitas non-est ponenda sine necessitate (plurality should not be posited without necessity) we will keep the scenario with the highest content of regularity because it allows us to achieve the same result with less complexity.

For this task, we are going to divide this subsection into four more:

- a regular model of PBS,

- a regular model of entanglement,

- an analysis about the interaction between the models, and

- a homology between entanglement, and the double slit experiment.

\subsubsection{A regular model of $P B S$}

In the PBS model of Figure 5, after flipping a coin, the rest of the model is deterministic, since for the same inputs $\{$ head, tail $\}$, it will always have the same outputs $\{|H\rangle,|V\rangle\}$, respectivelly. But, what happens if instead of tossing a coin we imagine a perfectly regular train of pulses inside it, generated based on a perfect clock like the one in Figure 26? 

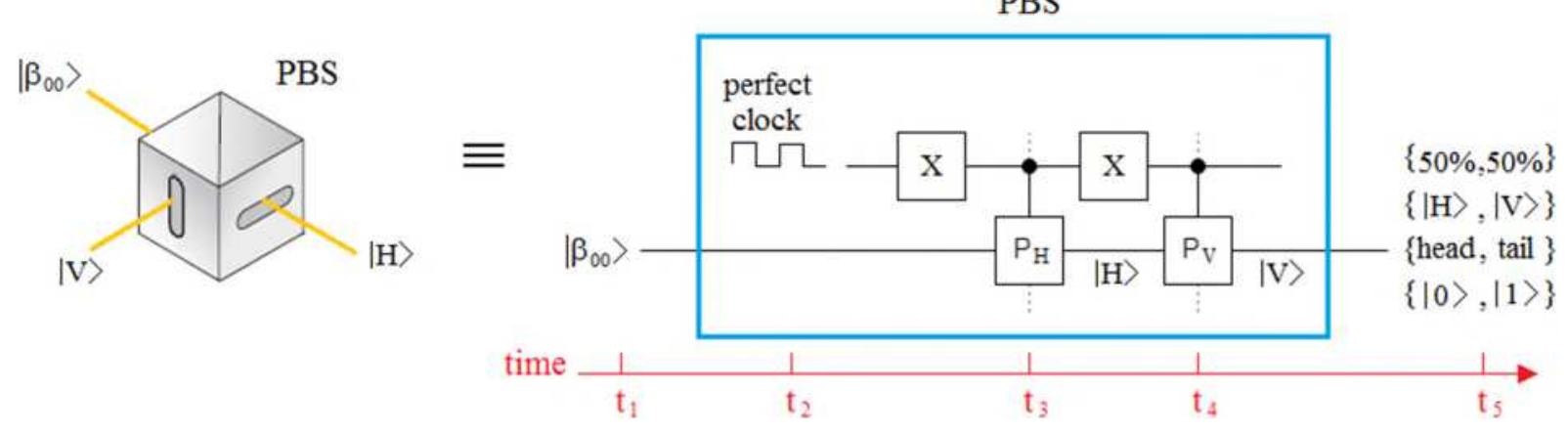

Figure 26. A regular model of the PBS.

In this case, the complete PBS model would be deterministic. Let us also imagine that the frequency of said clock is extremely high:

$f_{P}=\frac{c}{2 \pi l_{p}}=\frac{1}{2 \pi t_{p}} \cong 1.855 \times 10^{43} \mathrm{~Hz}$

where, $f_{\mathrm{P}}$ is the Planck's frequency, while $c \cong 300,000 \mathrm{~km} / \mathrm{sec}$ is the speed of light, $l_{\mathrm{P}}=$ $1.616255(18) \times 10^{-35} \mathrm{~m}$ is the Planck's length, and $t_{\mathrm{P}}$ is the Planck's time. Perhaps, it is an unattainable frequency by any human development, in the same way that it happens with the speed of light. In fact, this frequency is said to be the upper bound for the frequency of an electromagnetic ray, or cosmic rays, given that:

- Soft X-rays go from $3 \times 10^{17} \mathrm{~Hz}$ (with a wavelength of $10^{-9} \mathrm{~m}$, and $1.24 \mathrm{keV}$ ), to $3 \times 10^{18} \mathrm{~Hz}$ (with a wavelength of $10^{-10} \mathrm{~m}$, and $12.4 \mathrm{keV}$ ),

- Hard X-rays go from $3 \times 10^{18} \mathrm{~Hz}$ (with a wavelength of $10^{-10} \mathrm{~m}$, and $12.4 \mathrm{keV}$ ), to $3 \times 10^{19} \mathrm{~Hz}$ (with a wavelength of $10^{-11} \mathrm{~m}$, and $124 \mathrm{keV}$ ), and

- Gamma rays go from $3 \times 10^{19} \mathrm{~Hz}$ (with a wavelength of $10^{-11} \mathrm{~m}$, and $124 \mathrm{keV}$ ), to $3 \times 10^{20} \mathrm{~Hz}$ (with a wavelength of $10^{-12} \mathrm{~m}$, and $1.24 \mathrm{MeV}$ ), being the highest energy electromagnetic radiation.

This gives us a notion of the scale of the frequency involved in the model of Figure 26. In such a way that the only interaction of this model at a frequency equal to $f_{\mathrm{P}}$ with a classical entity such as the experimenter, who decides macroscopically when to apply the PBS in the path of Alice's beam is enough to generate a random result, independently of the random nature or not 
of an eventual internal model of the entanglement. This is due to a monumental mismatch in the order of the magnitudes involved between the internal model of the regular PBS, and in the macroscopic impossibility of Alice to precisely locate a certain point of her beam where to interpose the PBS. This would be more than enough to prevent both processes from being in phase, considering that one is classical and the other quantum. This tells us that the PBS could be perfectly regular and still the result of the measurement be random, although we still have another important element to analyze.

\subsubsection{A regular model of entanglement}

Although in the previous sections we put quantum measurement at the center of the problem, what we are really talking about is information, given that while we do not measure the elements of the entangled pair, they are found in a combination of states, but when we measure them, i.e., we try to inform ourselves of their situation, the first particle randomly takes one of the following determined states: spin-up or spin-down, which is accompanied by the end of the entanglement. Once the state of the first particle is established, and being the Bell's state of the type $\left|\beta_{00}\right\rangle=1 / \sqrt{2}\left(\left|0^{A}, 0^{B}\right\rangle+\left|1^{A}, 1^{B}\right\rangle\right)$, where the superscripts mean subsystem $A$ and $B$, the state of the second one is deterministic and instantaneously determined as the same of the first one, regardless of the distance between them. Since, we are dealing with information, the best tools to use are Entropy and Mutual Information ${ }^{[1]}$, through which we will carry out an entropic analysis of the problem posed in the previous sections.

For a better understanding of all the concepts to be developed from now on, we will organize this subsection through the following subsections:

- setup: we will use the concepts of Entropy and Mutual Information ${ }^{[1]}$ on the basis of which we will develop the entropic analysis. Particularly, Mutual Information is important as a metric to ponder the degree of correlation between the particles, 
- completely independent particles: they are particles without the slightest degree of correlation between them,

- classically-correlated particles: particles with a certain degree of mutual correlation,

- entangled particles: fully correlated particles (with the highest degree of correlation possible) which are called: maximally entangled particles, and

- projections of the three cases on a new internal model of entanglement: with important consequences on locality, causality, and instantaneity.

Setup: As a previous step for developing the Mutual Information ${ }^{[1]}$ as a metric, we need to begin with the definition of the density matrix of the subsystems (A, and B) treated individually,

$$
\rho^{A}=\rho^{B}=\frac{1}{2}(|0\rangle\langle 0|+| 1\rangle\langle 1|)=\frac{1}{2} I=\frac{1}{2}\left[\begin{array}{ll}
1 & 0 \\
0 & 1
\end{array}\right] .
$$

However, when a system is composed of two subsystems, its density matrix will depend on whether or not these subsystems are entangled. Therefore, for the non-entangled case, we will have:

$\rho^{A \cup B}=\rho^{A} \otimes \rho^{B}$,

but for the entangled case, the density matrix will be,

$\rho^{A \cup B} \neq \rho^{A} \otimes \rho^{B}$

This operator will depend on each of the three cases that will be analyzed, where Equation 54 represents the separable case, while, Equation 55 represents the non-separable case ${ }^{[1]}$. Now, with respect to the entropy, we will begin with the traditional definition introduced by von Neumann ${ }^{[1]}$, that is,

$S^{A}=S^{B}=-\operatorname{tr}\left[\rho^{A} \log \left(\rho^{A}\right)\right]=-\operatorname{tr}\left[\rho^{B} \log \left(\rho^{B}\right)\right]$,

where $\log (\bullet)$ means logarithm base 2 of $(\bullet)$, and $\operatorname{tr}(\bullet)$ is the trace of the square matrix $(\bullet)$. Then, replacing Equation 53 into Equation 56, yields,

$S^{A}=S^{B}=-\operatorname{tr}\left[\frac{1}{2}\left[\begin{array}{ll}1 & 0 \\ 0 & 1\end{array}\right] \log \left(\frac{1}{2}\left[\begin{array}{ll}1 & 0 \\ 0 & 1\end{array}\right]\right)\right]=1$. 
In the same way, for a composed system, the entropy is,

$S^{A \cup B}=-\operatorname{tr}\left[\rho^{A \cup B} \log \left(\rho^{A \cup B}\right)\right]$.

$S^{A \cup B}$ depends on the degree of correlation (completely independent, classically-correlated, and entangled) between both subsystems. Besides, in the classical and the quantum worlds, the correlations between the subsystems are those established by the additional information. In the case of composite quantum systems, the mutual information $S^{A \cap B}$ is introduced to quantify that additional information, allowing us to obtain the degree of correlation between both subsystems ${ }^{[1]}$,

$S^{A \cap B}=S^{A}+S^{B}-S^{A \cup B} \geq 0$.

Therefore, the entropy of the composite system $S^{A \cap B}$ indicates that the uncertainty of a state $\rho^{A \cup B}$ is less than the two subsystems $S^{A}$ and $S^{B}$ added together. In other words, $S^{A \cap B}$ tells us how much more information the composite system can store compared to the individual subsystems that compose it. Besides, $S^{A \cap B}$ measures the distance between the state $\rho^{A \cup B}$ and the non-entangled state $\rho^{A} \otimes \rho^{B}$ [1]. If states like those in Equation 54 are separable states, then the reduced density matrices of Equation 53 are pure states, thus the entropy of the state is zero. The nonzero value of the entropy of the reduced density matrix, therefore, is an indication of the possible existence of entanglement. Besides, the entropies of the subsystems $S^{A}$ and $S^{B}$ are equal to one in both cases (i.e., $S^{A}=S^{B}=1$ ); while the entropy of the composite system $S^{A \cup B}$ will have different values for each and every of the three mentioned cases. On the other hand, since both entropy and mutual information are scalar magnitudes ${ }^{[1]}$, from here on, we will use a scalar notation based on them which allows for a more practical treatment of the problem posed. Therefore, taking into account this criterion, and considering Equations 57 and 58, we will have,

$\mu_{A}^{2}=S^{A}=-\operatorname{tr}\left[\rho^{A} \log \left(\rho^{A}\right)\right]=1$, 
$\mu_{B}^{2}=S^{B}=-\operatorname{tr}\left[\rho^{B} \log \left(\rho^{B}\right)\right]=1$,

$\mu_{A \cup B}^{2}=S^{A \cup B}=-\operatorname{tr}\left[\rho^{A \cup B} \log \left(\rho^{A \cup B}\right)\right]$,

where $\mu_{A}, \mu_{B}$, and $\mu_{A \cup B}$ are the entropic values of the subsystems $\mathrm{A}$ and $\mathrm{B}$, and the composed system $\mathrm{A} \cup \mathrm{B}$, respectively. From Equation 59, we can obtain $S^{A \cup B}$,

$S^{A \cup B}=S^{A}+S^{B}-S^{A \cap B} \geq 0$.

The mutual information model as an effective coupling that reflects the experimental reality is:

$\mu_{A \cap B}^{2}=S^{A \cap B}=\mu_{A} \mu_{B} \kappa$,

where $\mu_{A \cap B}$ is the entropic-value of the mutual information, and $\kappa$ is a parameter which depends on the degree of correlation (completely independent, classically-correlated, and entangled) between both subsystems. Then, we can replace the elements of Equations 60, 61, 62, and 64 into Equation 63,

$\mu_{A \cup B}^{2}=\mu_{A}^{2}+\mu_{B}^{2}-\mu_{A \cap B}^{2}=\mu_{A}^{2}+\mu_{B}^{2}-\mu_{A} \mu_{B} \kappa$.

Table 4 shows Equation 65 for the three degrees of correlations between both subsystems, where each element of Equation 65 takes its respective value, including the parameter $\kappa$.

The only entropic-values that will have unalterable values regardless of the degree of correlation between the subsystems will be those belonging to the subsystems themselves, i.e., $\mu_{A}^{2}$ and $\mu_{B}^{2}$ of Equations 60 and 61 which are both equal to one, being this the reason why they do not appear in Table 4. Each of the three degrees of correlation will be analyzed below, where for the sake of simplicity, the subsystems are firstly expressed in qubits, being their respective entropy replaced by their corresponding entropic-value, where, said replacement together with a correct modeling of the Mutual Information will allow to highlight certain elements that would otherwise go unnoticed.

Table 4. Entropic-values in terms of the degree of correlations between both subsystems.

\begin{tabular}{c|c|c|c|c}
\hline \hline $\begin{array}{c}\text { Degree of } \\
\text { correlation }\end{array}$ & $\mu_{A \cup B}^{2}$ & $\kappa$ & $\mu_{A \cap B}^{2}$ & Graphic of sets \\
\hline
\end{tabular}




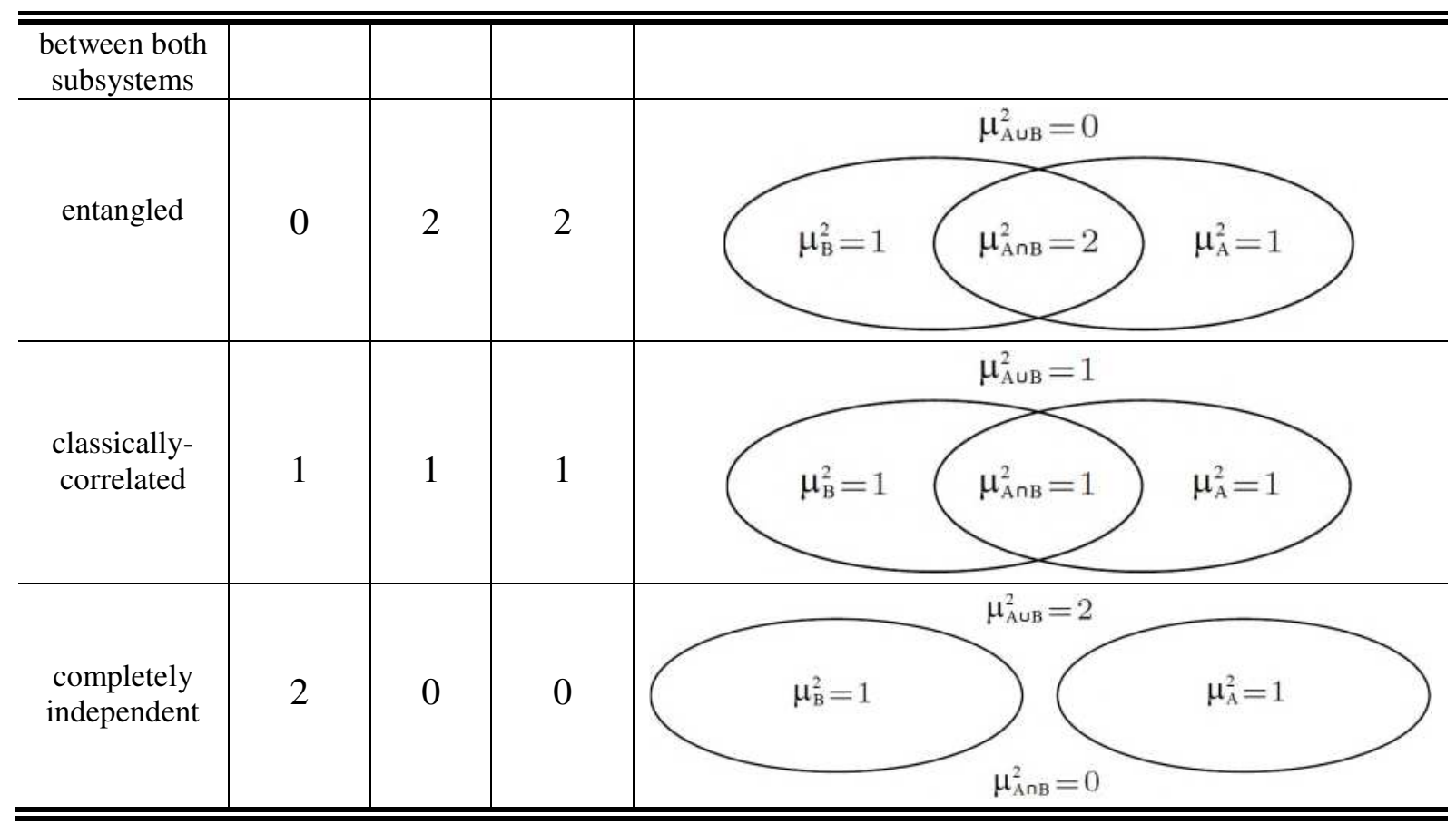

Completely independent subsystems: In this case, both subsystems have absolute and complete independence between them, i.e., $\rho^{A \cup B}$ is a Kronecker's product of density matrices like Equation 54. We can clearly see the independence of this case in Table 4. Therefore, there are no correlations between such subsystems. In the computational basis of $\mathrm{H}^{A} \otimes \mathrm{H}^{B}, \rho^{A \cup B}$ takes the following form,

$\rho^{A \cup B}=\frac{1}{4}\left(\left|0^{A}, 0^{B}\right\rangle\left\langle 0^{A}, 0^{B}|+| 0^{A}, 1^{B}\right\rangle\left\langle 0^{A}, 1^{B}|+| 1^{A}, 0^{B}\right\rangle\left\langle 1^{A}, 0^{B}|+| 1^{A}, 1^{B}\right\rangle\left\langle 1^{A}, 1^{B}\right|\right)=\frac{1}{4} I$,

where $I$ is a $4 \times 4$ identity matrix. Then, replacing Equation 66 into Equation 62, $\mu_{A \cup B}^{2}$ will be

$\mu_{A \cup B}^{2}=-\operatorname{tr}\left\{\frac{1}{4}\left[\begin{array}{llll}1 & 0 & 0 & 0 \\ 0 & 1 & 0 & 0 \\ 0 & 0 & 1 & 0 \\ 0 & 0 & 0 & 1\end{array}\right] \log \left(\frac{1}{4}\left[\begin{array}{llll}1 & 0 & 0 & 0 \\ 0 & 1 & 0 & 0 \\ 0 & 0 & 1 & 0 \\ 0 & 0 & 0 & 1\end{array}\right]\right)\right\}=2$.

This result can be seen in Table $4^{[1]}$, which introduced together with $\kappa=0$, and the results of Equations 60 and 61 into Equation 65, yields,

$\mu_{A \cup B}^{2}=\mu_{A}^{2}+\mu_{B}^{2}-\mu_{A \cap B}^{2}=\mu_{A}^{2}+\mu_{B}^{2}-\mu_{A} \mu_{B} \kappa=1+1-1 \times 1 \times 0=2$. 
It is evident that the entropic value of the mutual information is null, i.e., $\mu_{A \cap B}^{2}=0$, which implies null correlations between both subsystems for this case. ${ }^{[1]}$

Classically-correlated subsystems: In this case, it is possible to create correlations without obtaining entanglement for $\mu_{A \cap B}^{2} \neq 0$ as we can see in Table 4 for this case. For example, let us do this with the separable mixture of pure product states, where the density matrix will be,

$\rho^{A \cup B}=\frac{1}{2}\left(\left|0^{A}, 0^{B}\right\rangle\left\langle 0^{A}, 0^{B}|+| 1^{A}, 1^{B}\right\rangle\left\langle 1^{A}, 1^{B}\right|\right)=\frac{1}{2}\left[\begin{array}{cccc}1 & 0 & 0 & 0 \\ 0 & 0 & 0 & 0 \\ 0 & 0 & 0 & 0 \\ 0 & 0 & 0 & 1\end{array}\right]$.

Replacing Equation 69 into Equation 62, $\mu_{A \cup B}^{2}$ will be

$\mu_{A \cup B}^{2}=-\operatorname{tr}\left\{\frac{1}{2}\left[\begin{array}{llll}1 & 0 & 0 & 0 \\ 0 & 0 & 0 & 0 \\ 0 & 0 & 0 & 0 \\ 0 & 0 & 0 & 1\end{array}\right] \log \left(\frac{1}{2}\left[\begin{array}{cccc}1 & 0 & 0 & 0 \\ 0 & 0 & 0 & 0 \\ 0 & 0 & 0 & 0 \\ 0 & 0 & 0 & 1\end{array}\right]\right)\right\}=1$.

This result can be seen in Table $4^{[1]}$, which introduced together with $\kappa=1$ into Equation 65 , yields,

$\mu_{A \cup B}^{2}=\mu_{A}^{2}+\mu_{B}^{2}-\mu_{A \cap B}^{2}=\mu_{A}^{2}+\mu_{B}^{2}-\mu_{A} \mu_{B} \kappa=1+1-1 \times 1 \times 1=1$.

As we can see, $\rho^{A \cup B}$ of Equation 69 contains fewer terms than the completelly independent case of Equation 66 in the computational basis of $\mathrm{H}^{A} \otimes \mathrm{H}^{B}$, however, Equation 71 is more complete (all his terms are different from 0) than its counterpart of the completely independent case. In fact, the result of Equation 71 implies that $\mu_{A \cap B}^{2}=1$, bringing out a certain degree of correlation between both subsystems ${ }^{[1-3]}$, which has deep implications in the study of several types of correlated subsystems, not aligned with the central idea of this work.

Entangled subsystems: This is undoubtedly the most important case of the three, because of its projection on quantum communications. For this case, we are going to resort to Table 4. First, we will take one of the four possible Bell's states of Equation 28, specifically 
$\left|\beta_{00}\right\rangle=\frac{1}{\sqrt{2}}\left(\left|0^{A}, 0^{B}\right\rangle+\left|1^{A}, 1^{B}\right\rangle\right)$

Since it is a pure state ${ }^{[1]}$, we have

$\mu_{A \cup B}^{2}=S^{A \cup B}=0$.

This result can be seen in Table $4^{[1]}$, which introduced together with $\kappa=2$ into Equation 65 , yields,

$\mu_{A \cup B}^{2}=\mu_{A}^{2}+\mu_{B}^{2}-\mu_{A \cap B}^{2}=\mu_{A}^{2}+\mu_{B}^{2}-\mu_{A} \mu_{B} \kappa=1+1-1 \times 1 \times 2=0$.

Equation 74 shows that the degree of coupling, interaction, and mutual information between both subsystems is maximum. In fact, in a certain way Equation 74 also tells us about a conservation of the entropy through a balance of the involved entropic values, since this equation can be reorganized as follows:

$\mu_{A \cup B}^{2}=\mu_{A}^{2}+\mu_{B}^{2}-\mu_{A} \mu_{B} 2=\left(\mu_{A}^{2}+\mu_{B}^{2}\right)_{\text {originals }}-\left(\mu_{A} \mu_{B}+\mu_{A} \mu_{B}\right)_{\text {avatars }}=0$.

Equation 74 shows, on the one hand, the entropic values of the subsystems A and B as in the case of completely independent particles, i.e., $\mu_{A}^{2}$ and $\mu_{B}^{2}$, which will be called originals, while on the other hand, we have the entropic values of the mutual information, i.e., $\mu_{A} \mu_{B} 2$, which separated into two identical terms perfectly balance the equation, and we will call avatars. These avatars embody the maximum interaction or intersection between both subsystems like nothing else in the world of entanglement. It is precisely for this reason, that they are an instrument of interaction or intersection that in the next subsection will be placed physically, functionally and geometrically in the middle of the originals.

The avatars have identity and correspondence, that is, they are unambiguously associated with their corresponding original-entropic-value forming an indivisible original-avatar pair.

Moreover, the original values are inaccessible, unless the entanglement is destroyed (since they are immersed in an integrated context such as the Bell's elements of Equation 72), therefore, the avatars are too, i.e., if we do a local measurement ${ }^{[38,60]}$ on $\left|\beta_{00}\right\rangle$ for example on Alice's side, 
then the entanglement is destroyed and the avatars of Equation 75 immediately disappear, leaving one original in the hands of Alice and the other in the hands of Bob. The point here is that the avatars are equivalent to the originals from the operational point of view. This will also be seen in detail in the next subsection.

Projections of the three cases on a new internal model on entanglement: We are going to analyze the three degrees of correlation seen above but from the point of view of the spins or wave-functions involved. Having as a reference the Bell state $\left|\beta_{00}\right\rangle=1 / \sqrt{2}\left(\left|0^{A}, 0^{B}\right\rangle+\left|1^{A}, 1^{B}\right\rangle\right)$ again, at the top of Figure 27, we have the two possible configurations of the completely independent case, i.e.: $\left|0^{A}, 0^{B}\right\rangle$, and $\left|1^{A}, 1^{B}\right\rangle$. We arrived at these two possible configurations after locally applying a PBS on $\left|\beta_{00}\right\rangle$. As we can see, both spins are separated, in other words, there is no link between them and it is a configuration stripped of all dynamics, that is, it is a completely static case, without any other spins among the originals.

At the middle of Figure 27, two configurations of the classically-correlated case are presented: $\left|0^{A},+^{B}\right\rangle$, and $\left|+{ }^{A}, 1^{B}\right\rangle$. At a first glance, this case shows us a spin in the middle of the original spins. However, making an appropriate grouping of the spins by means of a gray balloon according to the corresponding membership of the intermediate spin for each configuration of this case, we see that although the degree of correlation increased with respect to the previous case, there are still two separate particles, giving rise to configurations without dynamics or physical link between both particles.

At the bottom of Figure 27, we analyze two possible configurations for the entangled particle case. As a result of all that has been said so far in this work, which is supported by countless 
a)

$\uparrow^{\left|0^{A}\right\rangle} \quad\left|0^{B}\right\rangle$

completely

independent

(static \& no link)

b)

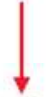

$\left|1^{A}\right\rangle$

$\left|1^{\lambda}\right\rangle\left|1^{\mathrm{B}}\right\rangle$

a)

$\left.\uparrow^{\left|0^{A}\right\rangle}\right|^{\left|0^{B}\right\rangle} \quad\left(\left|0^{A}\right\rangle\left|0^{B}\right\rangle+\left|0^{A}\right\rangle\left|1^{B}\right\rangle\right) \frac{1}{\sqrt{2}}=\left|0^{A}\right\rangle\left(\left|0^{B}\right\rangle+\left|1^{B}\right\rangle\right) \frac{1}{\sqrt{2}}=\left|0^{A}\right\rangle\left|+^{B}\right\rangle$

classically-

correlated

(static \& no link)

b)
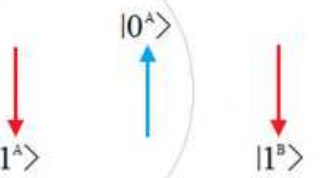

$\left(\left|0^{A}\right\rangle\left|1^{B}\right\rangle+\left|1^{A}\right\rangle\left|1^{B}\right\rangle\right) \frac{1}{\sqrt{2}}=\left(\left|0^{A}\right\rangle+\left|1^{A}\right\rangle\right)\left|1^{B}\right\rangle \frac{1}{\sqrt{2}}=\left|+^{A}\right\rangle\left|1^{B}\right\rangle$

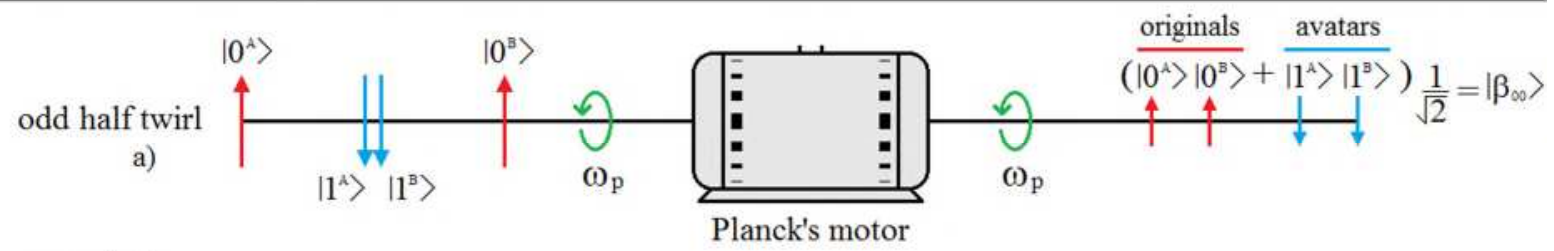

entangled

(dynamic \& linked)

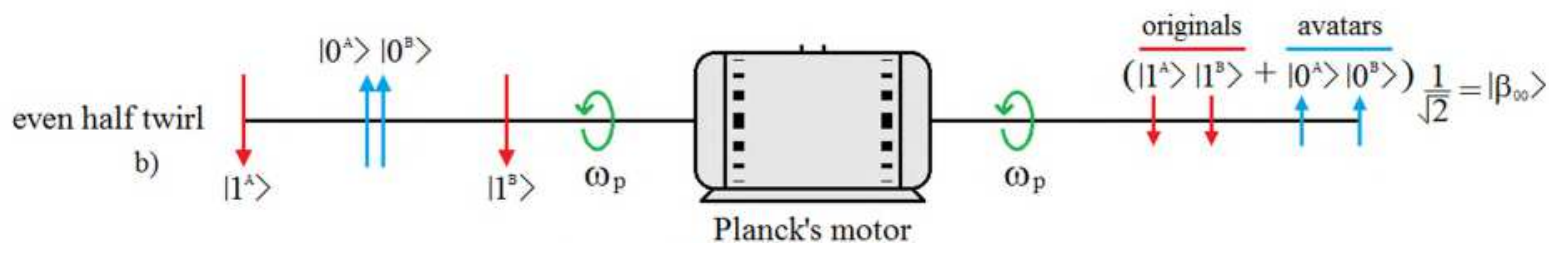

Figure 27. Representation of the three possible degrees of correlation between spins. Upper graph:

completely independent, a) $\left|0^{\mathrm{A}}>\right| 0^{\mathrm{B}}>$, b) $\left|1^{\mathrm{A}}>\right| 1^{\mathrm{B}}>$. It is a static case with no link between the spins.

Middle graph: classically-correlated, a) $\left.\left|0^{\mathrm{A}}>\right|+^{\mathrm{B}}\right\rangle$, b) $\left|+^{\mathrm{A}}>\right| 1^{\mathrm{B}}>$. It is another static case with no link between the spins. Lower graph: entangled, a) in an odd turn the originals are up $\left.\left|0^{\mathrm{A}}>\right| 0^{\mathrm{B}}\right\rangle$, and the avatars are down $\left|1^{\mathrm{A}}>\right| 1^{\mathrm{B}}>;$ b) in an even turn the originals are down $\left.\left|1^{\mathrm{A}}>\right| 1^{\mathrm{B}}\right\rangle$, and the avatars are up $\left|0^{\mathrm{A}}>\right| 0^{\mathrm{B}}>$. It is a dynamic case where the four spins are linked by a rigid bar in such a way that the structure performs quantum turns thanks to a Planck's motor at the Planck's frequency. 
laboratory experiences carried out around the world, each time we measure $\left|\beta_{00}\right\rangle$ we can obtain the two possible results mentioned repeatedly: $\left|0^{A}, 0^{B}\right\rangle$, and $\left|1^{A}, 1^{B}\right\rangle$. This clearly speaks to the underlying dynamics behind entanglement. In fact, the model presented here tries to reflect this dynamic as closely as possible.

The first step we will take is to functionally describe the first of the three fundamental tools behind the aforementioned dynamics. We will call this tool the Planck's motor. This motor allows its axis to perform quantum twirls, that is, the twirls go from $0^{\circ}$ to $180^{\circ}$ without going through the intermediate or prohibited angles similar to the case of prohibited orbitals in the electron shells of atoms, and it does so at the Planck's frequency $f_{\mathrm{P}}$ of the Equation 52.

The second tool needed to model this dynamics is a rotation shaft or bar that rotates without friction in vacuum and independent of the action of gravity, that links all the spins involved in each of the two possible configurations:

- odd half twirl: two original spins-up (red) to both ends, and two avatar spins-down (blue) at the middle, and

- even half twirl: two original spins-down (red) to both ends, and two avatar spins-up (blue) at the middle.

In these two configurations of the entangled case, we see two spins: the avatars in the middle of the two original spins. The avatars must be in the middle since as we saw in the analysis of the mutual information of Equation 75, they are interaction, intersection, or border spins. The avatars are the ones who exchange information and share mutual information since they interact without any distance between them, where one of them belongs to Alice and the other one to Bob. In fact, the avatars are the ones who really communicate.

The third and final tool needed to model this dynamic is an imaginary plane that rotates thanks to the shaft attached to the Planck's motor which contains all spins: originals and avatars, in other words, this plane contains $\left|\beta_{00}\right\rangle$. 
In this dynamic, a quantum measurement is understood as a sudden (instantaneous) stop of the entire structure in one of the two possible configurations: odd half twirl $\left(0^{\circ}\right)$ with outcomes $\left|0^{A}, 0^{B}\right\rangle$, and even half twirl $\left(180^{\circ}\right)$ with outcomes $\left|1^{A}, 1^{B}\right\rangle$, and never in intermediate or prohibited angles. In this context, we understand the synchronization of nonlocal outcomes, before and after quantum measurement, as the rotation of the structure composed by the Planck's motor axis, and therefore, the plane that contains all the spins, that is, there is no transmission of information, according to the standards of traditional communication systems in which something travels from point A to B, but rather a mechanical twirl of a single structure, which is why there is no synchronization effort. It is not that something travels from A to B fasterthan-light, but that A and B are forming part of something bigger, they are integrated, so the synchronization is instantaneous without violating Special Relativity. In fact, there is no A and $\mathrm{B}$, but $\mathrm{AB}$. The entanglement does not constitute a communication system since there is never a communication at any transmission speed. For this reason, it is not necessary to resort to faster-than-light speed to instantly synchronize non-local outcomes. There is no such thing as two sides at the same time, but a single thing occupying a greater space through a link with a dilated, integrated or linked locality, that is, a kind of macro-particle.

Next, we will delve into detail about this particular aspect: the locality. Moreover, we will analyze why avatars are much more than a simple binder, representing an equivalent to the transmission that connects the engine with the wheels in a car. In fact, they are the icon of the dynamics of entanglement, as they are the first to disconnect (they disappear from the scene) when the structure stops.

The length of the axis is part of the distribution process of the EPRs prior to the measurement. In fact, and in order to get into the analysis of the locality, let us suppose that the axis is like a single train track that connects NY with LA. The portion of the track near NY is local to NY, the same happens with the one near LA, but the NY portion of track is not local to LA, although 
the track is only one. The phenomenon is non-local, but it can also be interpreted as an expansion of the locality thanks to the process of distribution of the EPRs, or extension of the axis. At this point, if there is no transfer of the state from A to B and vice versa, but simply a common axis that rotates for all the elements at the same time, being this a single rigid structure, then there is no transmitter, no receiver, nor channel. This single fact, represents in itself, a change of the communicational paradigm. In fact, the channel has a length equal to zero since it is the distance between the avatars, which are located exactly at the same point, and this channel will only be in effect as long as the entanglement exists.

Making the focus on the locality, we resort to Figure 28, which only presents the configurations (a) of the three cases of Figure 27. For the completely independent case, both their original and equivalent configurations look identical in the two central columns of Figure 28, where there is no overlap between the locality globes of both particles. In contrast, for the classicallycorrelated case, there appears to be such an overlap with a shared spin-down (blue) in the middle. However, that spin only belongs to Bob. Therefore, exercising the correct treatment of the equations found in the lower part of the locality globes, we see that there is separability in two well-defined particles: $\left|0^{A}\right\rangle$, and $\left|+{ }^{B}\right\rangle$, which are as separate as the completely independent case, as its equivalent configuration shows us. It is an intermediate instance (between the classically-correlated case and the entangled one) since they are canonically overlapped, however they are factorisable, separable or distinguishable.

On the other hand, at the bottom of Figure 28, both entangled particles have overlapped their respective locality balloons (grays) with two spin-down avatars (blue) in the intersection of those balloons, where finally these balloons constitute a single integrator. This is equivalent to a single wave-function (green) or single locality corresponding to a single equivalent macroparticle. The overlapping of the locality globes tells us of an overlap of the same localities, forming a single dilated locality. 


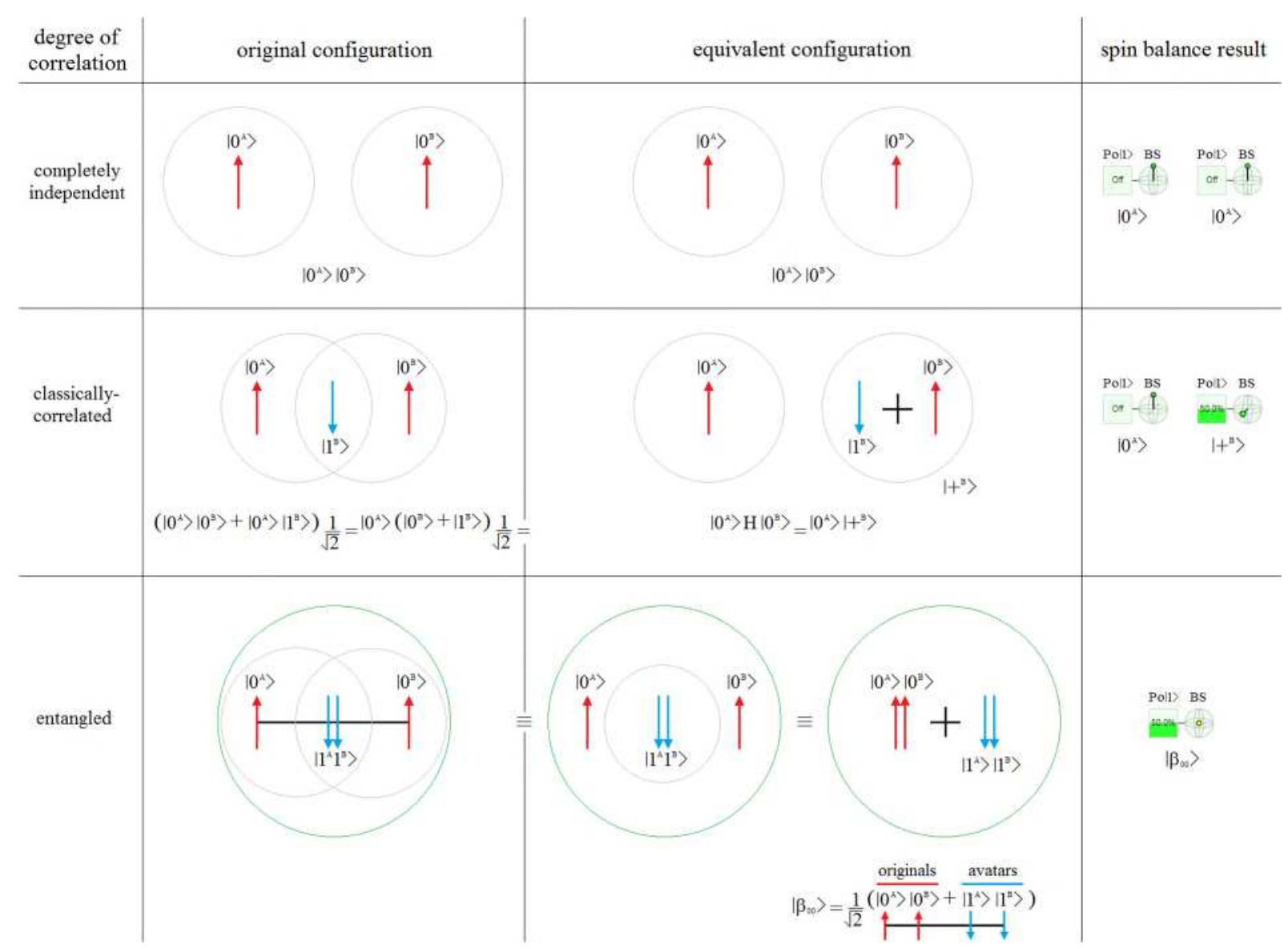

Figure 28. Locality balloon diagrams for the three degree of correlation, with the equivalent configurations, and the spin balance results. In the case of entanglement, it can be seen how the individual localities overlap, constituting a unified and dilated locality, which justifies local control of non-local outcomes.

On the other hand, since:

a) one of the two avatars corresponds to Alice and the other one to Bob,

b) Alice's locality contains her original spin and her respective avatar, in the same way as in the case of Bob, and

c) both avatars are in the middle, in the same place, with no separation between them, even though the original spins are on opposite sides of the universe,

it is clear that it is the avatars that stretch the individual localities until they overlap and finally integrate into only one, reproducing the behavior of an indivisible macro-particle. In fact, if the 
avatars did not exist, then both localities would never overlap (as in completely independent and classically-correlated) and there would be no entanglement.

This model explains instantaneity, since synchronization does not imply a communication effort between Alice and Bob to coordinate their results at all, as it is the same result associated with a simple rotation of an axis of a rigid structure. This automatically allows both outcomes to be synchronized without confronting Special Relativity with Quantum Mechanics, since nothing travels faster than light to instantly synchronize both, because from the point of view of entropy, there is no such thing as both.

By getting the PBS in the path of Alice's beam, the final state does not depend on a transfer of information between $\mathrm{A}$ and $\mathrm{B}$, but on where the plane stops when measuring, and since it is a single rigid object, both of them are naturally in sync. There is no need for additional communication between A and B to synchronize the outcomes through a classical auxiliary channel, there are no roles of $\mathrm{A}$ and $\mathrm{B}$ as sender and receiver but only $\mathrm{AB}$ inside the entanglement, that is, it is a single macro-particle with a macro-wave-function, which has zero spin (small yellow circle) located in the center of the Bloch sphere as shown in the last column of Figure 28. This represents a perfect balance or conservation of the spins within the common locality globe (green).

\subsubsection{Analysis about the interaction between the models}

In the previous subsections, it was established that the internal models of the PBS and the entanglement can be individually perfectly regular, each with a perfect internal clock, and yet the quantum measurement continues to be random. In this subsection, we are going to up the ante and assume that not only are the internal model of the PBS and the entanglement individually regular, but that they are simultaneously regular, to the point that they can be the same internal clock and in perfect phase, and yet the quantum measurement can be completely random due to the human intervention only.

Figure 29 shows with Cartesian axes: a) the internal model of the PBS in the three upper axes, 
and b) the internal model of the entanglement in the four lower axes, which represent the internal state of each of the spins that constitute the internal model of the Bell State $\left|\beta_{00}\right\rangle$. Each

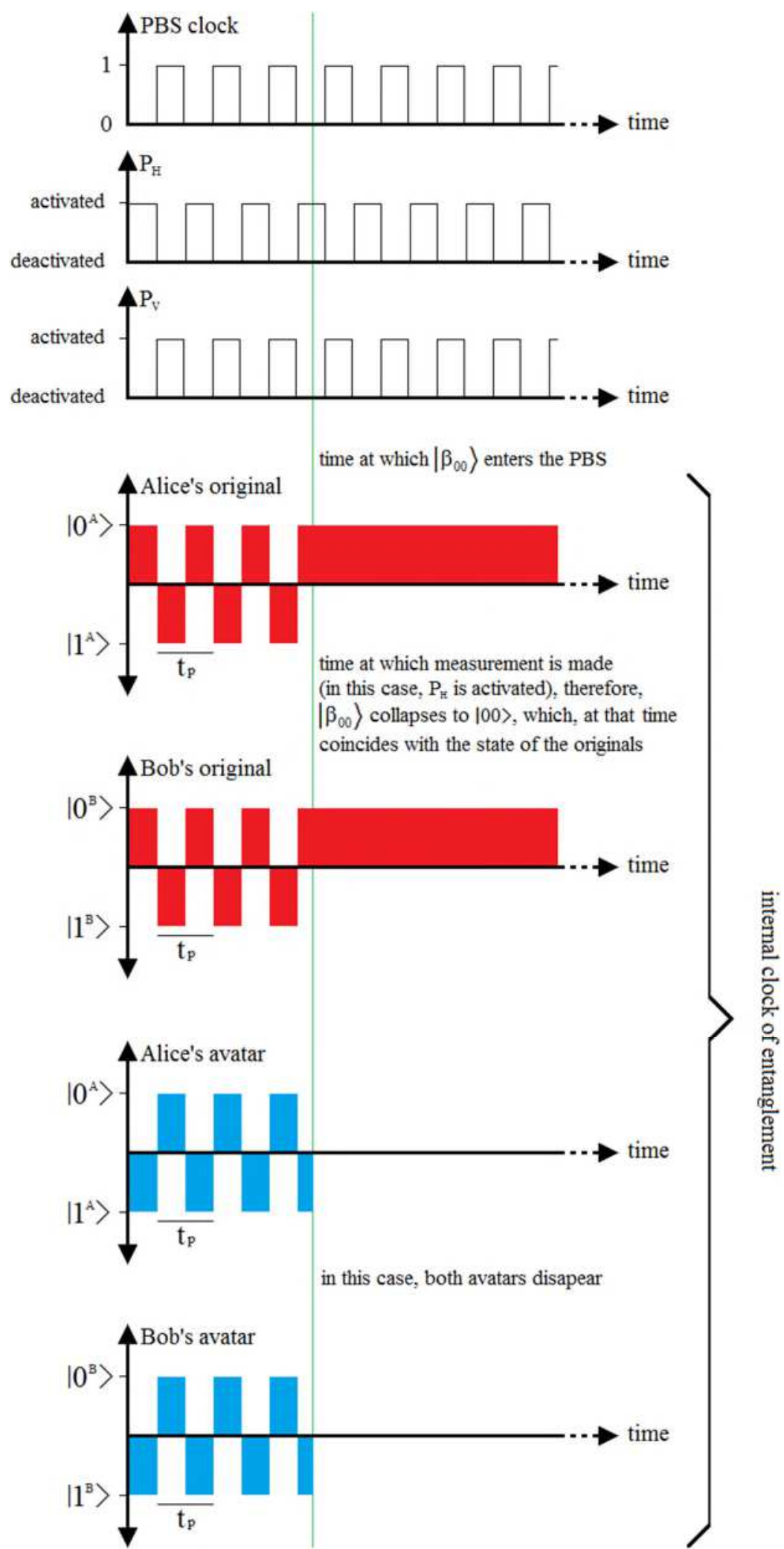

Figure 29. Details of the internal clocks of the PBS and entanglement. 
half pulse of the PBS internal clock (Figure 26) corresponds to each half-turn of the plane of the configurations in the lower part of Figure 27, such that in each odd half twirl, the plane is at $0^{\circ}$, while in every even half twirl, the plane is at $180^{\circ}$.

Figure 29 is in perfect coordination with the internal pulses of the PBS of Figure 26, and with the lower part of Figure 27. When the internal clock of the PBS of Figure 26 is at 0 , said 0 enters the inverter $\mathrm{X}$ and becomes a 1 , activating the $\mathrm{P}_{\mathrm{H}}$, and deactivating the $\mathrm{P}_{\mathrm{V}}$ by action of the second inverter X. In this case, in the configuration (a) of the lower part of Figure 27, both original spins are spin-up, while their respective avatars are spin-down, as shown by the first half pulse of each one of the final four axes of Figure 29. On the other hand, when the internal clock of Figure 26 is at 1, the opposite happens, which corresponds to the configuration (b) at the bottom of Figure 27, and to the second half pulse of each of the four final axes in Figure 29. The important thing happens when the PBS actually gets in the way of Alice's beam, in which case a collapse of the wave-function occurs. If at the time of collapse the originals are at $|0\rangle$, then they will remain like that, while the avatars disappear. Instead, if the collapse happens when the original spins are at $|1\rangle$, they will stay that way, while the avatars will again disappear. Finally, Figures 27 (lower part) and 29 show that local or non-local hidden variables are not necessary for the aforementioned instantaneous synchronization of non-local outcomes. Only the original spins and their avatars are required in the entropic lay-out of the Figure 27 (lower part).

We conjecture that God does not play dice: Having performed detailed internal modeling of both the PBS and the entanglement, where both elements can be perfectly regular and yet the quantum measurement can continue to be random, we can establish that these models undermine the very foundations of the randomness, not its existence but its origin, since the inherent regularity of both models is perfectly feasible, and the randomness is exclusively reserved at the moment in which the experimenter decides to apply the PBS. On the other hand, 
the literature holds measurement directly and fully responsible as if randomness were something intrinsic to it. Instead, these models show us that both the entanglement (as regards the alternation of their spins) and the PBS could be perfectly regular elements and the sole intervention of a man choosing the moment to apply the PBS on the entanglement could return an overall result of random characteristics.

There could be a single perfect clock of the quantum universe, something like a metronome of the music of said universe that synchronizes everything, which within the PBS alternates 0s and 1s, and within $\left|\beta_{00}\right\rangle$ alternates $|00\rangle$ and $|11\rangle$, and even so the location of the PBS (taking into account the speed of the photons in the medium used, the number of photons per second emitted by the pump, the number of entangled pairs traveling through each branch, and the trajectory of each entangled photon by its respective branch of Figure 4) decides the result, that is, God does not play dice but randomness is the absolute responsibility of a man, that is, of the experimenter. In fact, the frequency of emission of entangled photons by the pump+BBO configuration depends on the power of the pump and generally this source provides a maximum production rate of $23010^{6}$ pairs (230 Mpairs) of energy time entangled photons per second. That is to say, the frequency of the clock behind the entanglement and the PBS is much higher than that of the occurrence of entangled photons in each branch. Furthermore, the frequency of the laser is not stable, so the frequency of appearance of the entangled photons at the doors of the PBS will not be either. This generates an arrhythmia that triggers another contribution to the randomness associated with the measurement of the entanglement, in contrast to the internal clock of the PBS and the entanglement, which is perfectly regular.

Based on this, we apply a discard as part of the procedure applied here to identify the true culprit of the randomness. Said discarding consists of analyzing what values each of the terms of Equation 51 can take, and which ones they cannot. That is, if in Equation 51 we associate randomness with a result equal to 1 , in such a way that with only one of its three members being 
1, said equation complies with the randomness observed during each quantum measurement process carried out in laboratories around the world, and the PBS model can be regular (RIPBS $=0$ ), and that of the entanglement also $\left(\mathrm{RI}_{\mathrm{E}}=0\right)$, then, the complete responsibility for the randomness can only be due to human intervention, since by the difference in magnitudes and characteristics (classical vs. quantum) of the elements involved, human intervention can never be regular $\left(\mathrm{RI}_{\mathrm{HI}} \neq 0\right)$. That is, it is preferable to associate randomness to the exclusive result of human intervention since this result is more elegant, simple, ordered and regular than the possibility that each quantum element in the quantum universe has its own independent random dynamics.

In fact, human intervention is not just random, it is much more than that, it is the icon of the classical world trying to intervene in the quantum world, like a bull in a china shop, and this is due to two reasons:

a) human intervention in the problem of quantum measurement is equivalent to trying to select or separate with a finger a particular molecule in a gas with $10^{23}$ molecules enclosed in a cubic container with a side of $1 \mathrm{~cm}$. Absurd, right? The selection instrument, in this case a human finger (classical) is not the appropriate tool on the appropriate scale for that particular task. This is the root of the problem, that is, the experimenter makes an imprecise execution decision given the difference in scale in the dimensions involved. In other words, there is a scale incompatibility between the frequency of the internal clock of the entanglement and the PBS, that is, $f_{P}$ (and hence the Planck length $l_{P}$ ), the number of entangled photons per second in each branch, and the location of PBS by the experimenter. This difference in scale or resolution is due to the fact that the finger is much larger than many clock pulses in the quantum world. Therefore, as much as man could pinpoint PBS with picoscopic precision, there would still be mismatch and randomness, not to mention that

b) a man cannot look inside the entanglement or the PBS to know what phase they are in to correctly adjust or synchronize the phase, and select a particular state to apply the PBS. In 
fact, it is not possible to intervene in an intermediate instant of each half cycle of the clock of the quantum world that synchronizes everything, since $t_{p}$ is about the minimum quantum expression of time, therefore, Figure 29 is, in a certain form, only symbolic.

In other words, any man-made instrument intended to accurately measure or intervene in the quantum world will be so crude in its conception, application, resolution, and precision that it will inevitably lead to randomness. On the other hand, in the same way that Figure 27 explains post-distribution instantaneity, i.e., instantaneous synchronization as a result of quantum measurement without the need for FTL-type information transmission, Figure 29 explains that it is possible a complete regularity of the quantum world and still obtain a random result as a consequence of the application of a quantum measurement.

Quantum horologium unique: In Figure 29, the pre-existence of a clock inside a PBS (where the latter is basically a crystal) prior to the application of said PBS in the path of $\left|\beta_{00}\right\rangle$ may seem strange, but as we have seen so far, we are talking about a single clock of the quantum world, therefore, the phase of selection of the polarizers internal to the PBS is given in relation to that single clock, the same one that governs the internal rhythm in the dynamics of the entanglement. The PBS itself resorts to that clock when it is required to do so. In other words, since the measurement only becomes effective at the time of the arrival of $\left|\beta_{00}\right\rangle$ to the PBS, then the activation of the appropriate polarizers within the PBS only takes place after said PBS resorts to the mentioned clock, that is, after that it resorts to the quantum nature of its particles. Thus, the quantum world could be absolutely regular, synchronized by that single clock (the Planck's clock) and yet the result of the measurement would be random due to the random moment at which $\left|\beta_{00}\right\rangle$ enters the PBS, neglecting any internal delay it may have placed in it. In Figure 29 a symbolic moment of incidence of $\left|\beta_{00}\right\rangle$ in the PBS is shown, since the effort of locating said PBS has an imprecision enormously greater than the wavelength of the internal clock of the PBS and entanglement. Although in reality we speak of a symbolic instant because 
it is unattainable by men, since this last wavelength, the Planck length $l_{P}$ is the elementary unit of quantum length, in the same way that $t_{P}$ is the minimum expression of time in the quantum world, that is, it represents the quantum of time. There is no possibility of accessing an intermediate instant of any pulse in Figure 29. In this order of things, $E_{P}=\omega_{P} \mathrm{~h}$, is the minimum energetic expression of nature, where $\omega_{P}=2 \pi f_{P}$, and $\mathrm{h}$ is the reduced Plank constant.

Other contributors to randomness are: a) phase and frequency instability of the laser, since there is no perfect laser, which also impacts at the time of arrival of the $\left|\beta_{00}\right\rangle$ to the PBS, as does, b) the location of the PBS in the path of the beam. That is, randomness responds to a sum of factors, all due to human intervention.

Finally, there is an underlying clock in the bowels of the quantum world with which the whole universe coordinates and that started with the creation of said universe, and if at some point the speed of light was not the same as it is now, then said clock worked accordingly.

Bandwidth and channel capacity of entanglement: If we observe the four lower axes of Figure 29, all due to the internal model of the entanglement, we can see that they are perfectly rectangular pulses with rising and falling flanks at $90^{\circ}$ which shows the instantaneity of synchronism of non-local outcomes as a consequence of quantum measurement. Based on Fourier analysis ${ }^{[61]}$, these pulses have spectral components of null and infinite frequencies. Therefore, the entanglement will have infinite intrinsic bandwidth. This does not mean at all that a communication system based on entanglement has unlimited bandwidth, in the same way that it cannot be instantaneous either.

In any man-made system, the strength of that system is that of its weakest component. An entanglement-based communication system is no exception. Many elements in solidarity with entanglement and that are part of the communication protocol are degrading its intrinsic bandwidth, for example: the laser bandwidth, the bandwidth of the optical fiber that distributes 
the entangled pairs, and so on.

Strictly speaking on quantum communication, the lower parts of Figures 27 and 28 show zero distance between avatars. This implies the absence of a channel between them, which is reduced to a point. Therefore, what a transmitter deposits at that point is perfectly and exactly equal to what a receiver receives from that point, i.e., what you put at that point is equal to what you get from that point (WYPWYG). Then, that channel has infinite bandwidth and null latency, as a consequence of this, it is more appropriate to say that the avatars share information in that pointchannel, instead of transmitting information through that point, then this communication technology is a non-channel technology. In fact, the distribution of the EPR pair can be interrupted by any factor that blocks the optical link, or any other means used for distribution, but once the distribution is established, nothing will interrupt the entanglement, since the avatars are all together at the same point, therefore, a link based on entanglement does not suffer fading, vanishing, no-thing interrupts it or intercepts it, it does not need antennas, or high power transmission systems. The entire universe is reduced to that point. There is no dark side of the moon for entanglement. Only one thing ends with entanglement, quantum measurement or decoherence. Also, it is important to mention that entanglement is a monogamous process. ${ }^{[1-3]}$ A direct consequence of everything mentioned here is that since there is no channel between avatars, there is no channel noise, since noise cannot be introduced into a channel that does not exist. So from Information Theory ${ }^{[62]}$, we know that with infinite bandwidth and zero noise, the intrinsic channel capacity of the entanglement is also infinite. Unfortunately, a communication system based on entanglement could never take advantage of these formidable virtues.

The quantum world self-organizes into octaves: That organization is essential for all the options present in each experiment to exist. For example, in Figure 17, the controller injects two different pulse trains into the respective rotatable polarizers, so the options are:

- the controller injects a pulse train 0101 into the rotatable polarizer of $\mathrm{q}[0]$, and

- the controller injects a 0011 pulse train into the rotatable polarizer of $\mathrm{q}[1]$. 
As we have seen, when the controller in Figure 17 sends zeros to both rotatable polarizers at the same time, both $\mathrm{P}_{\mathrm{H}}$ are activated, and consequently Bob directly reconstructs the teleported qubit without applying any other unitary transforms other than the identity matrix. Then, in Figures 19 and 20, we see that the $\mathrm{P}_{\mathrm{H}}$ of $\mathrm{q}[0]$ retains $50 \%$ of the state that passes through it, at the same time canceling that same percentage in $q[1]$, in such a way that the $\mathrm{P}_{\mathrm{H}}$ of $\mathrm{q}[1]$ retains $50 \%$ of $50 \%$, that is, it blocks another $25 \%$. Both of them hold together $75 \%$ of everything that goes through them on Alice's side, allowing Bob only to receive the remaining $25 \%$. The selforganization in octaves can be seen in two very clear aspects:

- the pulse train of $\mathrm{q}[1]$, is a disambiguous sub-harmonic of the pulse train of $\mathrm{q}[0]$, of exactly half its frequency, i.e., in a generic way, $f_{\text {sub-harmonic }}(i+1)=f_{\text {sub-harmonic }}(i) / 2$, where $i$ is the degree of the sub-harmonic one, being $i=0$ for the original pulse, and

- the $\mathrm{P}_{\mathrm{H}}$ of $\mathrm{q}[1]$ retains half of what the $\mathrm{P}_{\mathrm{H}}$ of $\mathrm{q}[0]$ retains.

All of this happens identically inside a PBS.

If in Figure 17, there was a single clock pulse in the quantum world, then, how can we explain the generation of the four options? Clearly, $\mathrm{f}_{\mathrm{P}}$ is the basal rate, the rest is organized into subharmonics. However, in the entire period of the sub-harmonic (the one with the lowest frequency), both trains of pulses have the same number of 1s:

- 0101 , in 2 periods $\left(t_{\mathrm{P}}\right)$ of the basal rate $\left(\mathrm{f}_{\mathrm{P}}\right)$ there are $21 \mathrm{~s}$, and

- 0011 , in 1 period $\left(2 t_{\mathrm{P}}\right)$ of the first sub-harmonic with frequency $\left(f_{\mathrm{P}} / 2\right)$ there are $21 \mathrm{~s}$ too.

For the case of quantum secret sharing in Figure 23(b), the controller injects three pulses with the following results:

- the original pulse of the clock 01010101 enters the rotatable polarizer of $\mathrm{q}[0]$,

- the first disambiguous sub-harmonic 00110011 enters the rotatable polarizer of $\mathrm{q}[1]$, and

- the second disambiguous sub-harmonic 00001111 enters the rotatable polarizer of q[2].

The $\mathrm{P}_{\mathrm{H}}$ of $\mathrm{q}[0]$ retains $50 \%$ of the state that passes through it, the $\mathrm{P}_{\mathrm{H}}$ of $\mathrm{q}[1]$ retains $50 \%$ of $50 \%=25 \%$, 
while the $\mathrm{P}_{\mathrm{H}}$ of $\mathrm{q}[2]$ retains $50 \%$ of $25 \%=12.5 \%$. The three $\mathrm{P}_{\mathrm{H}}$ hold together $87.5 \%$ of everything that goes through them on Alice's side, allowing Bob only to receive the remaining $12.5 \%$.

The case of Figure 24(b) for entanglement swapping is identical in its characteristics and results to those of Figures 19 and 20 for teleportation, retaining $75 \%$ on Alice's side and keeping $25 \%$ on Bob's side. However, an interesting case is represented by the quantum repeater in Figure 25(b) where a controller injects four pulses with the following results:

- the original clock pulse 0101010101010101 enters the rotatable polarizer of $\mathrm{q}[2]$,

- the first disambiguous sub-harmonic 0011001100110011 enters the rotatable polarizer of q[3],

- the second disambiguous sub-harmonic 0000111100001111 enters the rotatable polarizer of $\mathrm{q}[0]$, and

- the third disambiguous sub-harmonic 0000000011111111 enters the rotatable polarizer of q[1], where the respective $\mathrm{P}_{\mathrm{H}}$ produces the following results:

- the $\mathrm{P}_{\mathrm{H}}$ of $\mathrm{q}[2]$ retains $50 \%$,

- the $\mathrm{P}_{\mathrm{H}}$ of $\mathrm{q}[3]$ retains $50 \%$ of $50 \%=25 \%$,

- the $\mathrm{P}_{\mathrm{H}}$ of $\mathrm{q}[0]$ retains $50 \%$ of $25 \%=12.5 \%$, and

- the $\mathrm{P}_{\mathrm{H}}$ of $\mathrm{q}[1]$ retains $50 \%$ of $12.5 \%=6.25 \%$.

The four $\mathrm{P}_{\mathrm{H}}$ together retain $93.75 \%$ on Alice's side and hold only $6.25 \%$ on Bob's side.

To summarize, as we have seen, in order to generate the necessary options for teleportation, entanglement swapping, quantum secret sharing and quantum repeater, nature organizes itself in octaves within all of PBS.

\subsubsection{A homology between entanglement and the double slit experiment}

The double slit experiment ${ }^{[63]}$ is commonly associated with the wave-particle duality of electrons. However, this experiment can be implemented with water waves in a pond, photons, and so on.

Figure 30 represents an implementation of this experiment carried out with photons. In the 
lower part of this figure the experiment begins with the emission of photons by a single particle source. These photons pass through both slits of the double slit assembly generating two rays that, when converging together at different points on the screen, generate two types of patterns. The distribution of photons on the screen when both slits are open is given by $\psi_{A \cup B}(x)=c\left(\psi_{A}(x)+\psi_{B}(x)\right)$,
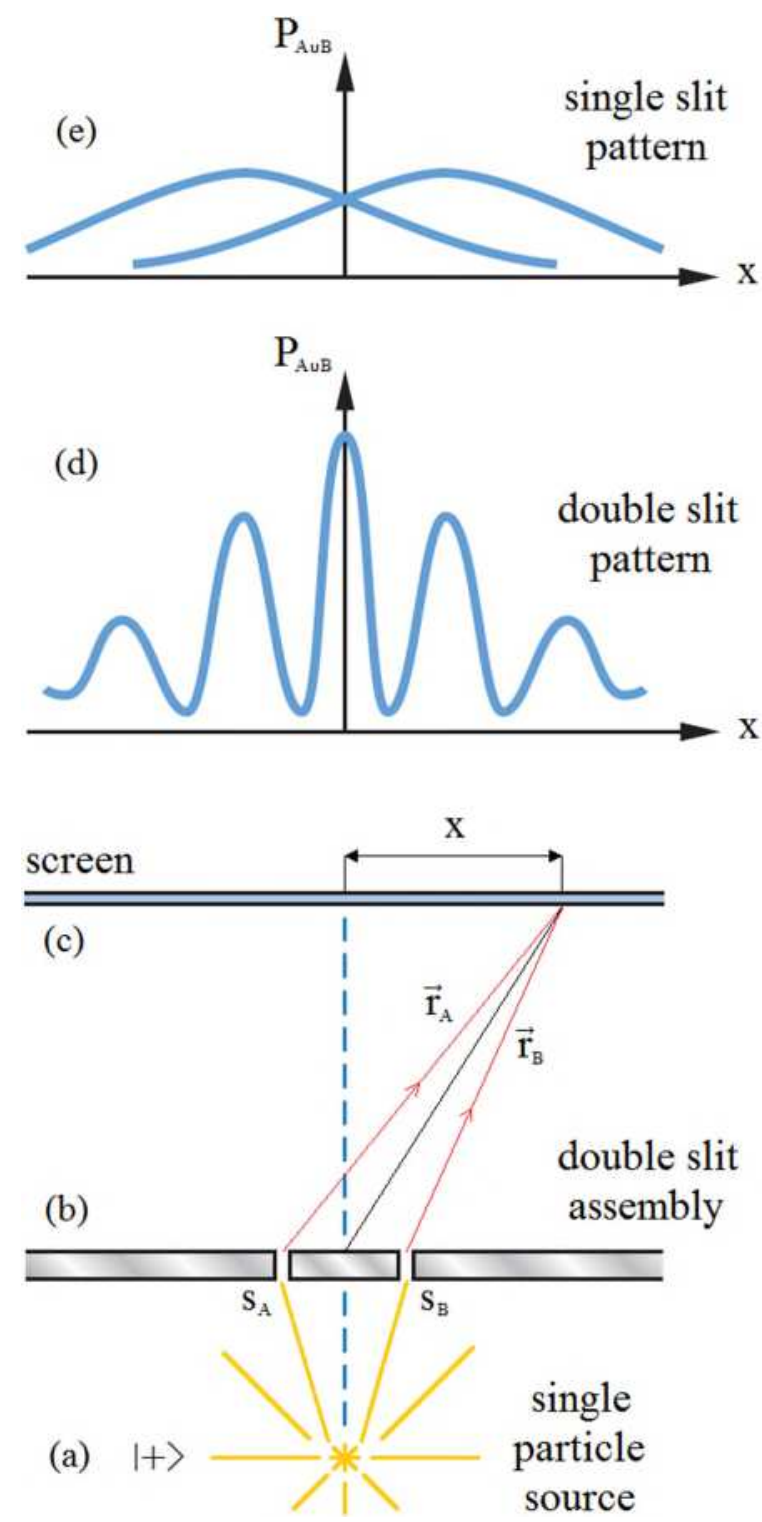

Figure 30. Double slit experiment. The particles generated by a single particle source (a) traverse both slits $\left(\mathbf{S}_{\mathrm{A}}\right.$ and $\left.\mathbf{S}_{\mathrm{B}}\right)$ of a double slit assembly (b). If no detector is used, then the pattern observed on screen (c) is the one shown in figure (d), but if a detector is located in either of the two slits, then the observed pattern is that of figure (e). 
where $c$ is a constant of normalization, $\psi_{A}(x)$ is the photon distribution on the screen when slit $\mathrm{A}$ is open and slit $\mathrm{B}$ is closed, while $\psi_{B}(x)$ is the photon distribution on the screen when slit B is open and slit A is closed. The theory of interference of waves tells us that the probability distribution of the photon position on the screen when both slits are open is

$$
\begin{aligned}
P_{A \cup B}(x) \cong\left|\psi_{A}(x)+\psi_{B}(x)\right|^{2} & =\left|\psi_{A}(x)\right|^{2}+\left|\psi_{B}(x)\right|^{2}+2\left|\psi_{A}(x)\right|\left|\psi_{B}(x)\right| \cos \left(\frac{2 \pi}{\lambda}\left(\begin{array}{ll}
\mathbf{u r} & \mathbf{u r} \\
r_{A} & -r_{B}
\end{array}\right)\right) \\
& =\left|\psi_{A}(x)\right|^{2}+\left|\psi_{B}(x)\right|^{2}+2 \operatorname{Re}\left(\psi_{A}^{*}(x) \psi_{B}(x)\right)
\end{aligned}
$$

where $\lambda$ is the wavelength of the incident wave, $\operatorname{Re}(\bullet)$ means real part of $(\bullet)$, and $(\bullet)^{*}$ is the complex conjugate of $(\bullet)$. However, if the use of detectors in slits A and B is alternate, so that one slit is always closed, the probability distribution of the photon position on the screen will be

$$
P_{A \cup B}(x) \cong\left|\psi_{A}(x)\right|^{2}+\left|\psi_{B}(x)\right|^{2}
$$

That is, the interference factor just disappears as well as the double slit effect. Therefore, if we redefine Equation 77 in terms of each of the individual contributions to the overall pattern observed on the screen, we have to

$$
P_{A \cup B}(x) \cong\left(\left(\left|\psi_{A}(x)\right|^{2}+\left|\psi_{B}(x)\right|^{2}\right)_{\text {singlesslit }}+\left(\operatorname{Re}\left(\psi_{A}^{*}(x) \psi_{B}(x)\right)+\operatorname{Re}\left(\psi_{A}^{*}(x) \psi_{B}(x)\right)\right)_{\text {interference }}\right)_{\text {double-slit }}
$$

From Equation 79, we can see that it bears a great similarity to the Equation 75, which we reordered appropriately resulting in

$$
\begin{aligned}
\mu_{A \cup B}^{2} & =\left(\mu_{A}^{2}+\mu_{B}^{2}\right)_{\text {originals }}-\left(\mu_{A} \mu_{B}+\mu_{A} \mu_{B}\right)_{\text {avatars }} \\
& =\left(\left(\mu_{A}^{2}+\mu_{B}^{2}\right)_{\text {single-slit }}-\left(\mu_{A} \mu_{B}+\mu_{A} \mu_{B}\right)_{\text {interference }}\right)_{\text {double-slit }}
\end{aligned}
$$

The similarity between these two equations provides clear evidence that:

- the original spins keep a complete correspondence with the individual opening of the slits,

- avatars embody interference,

- double slit experiment appears to be the result of an entanglement, and

- entanglement appears to be the result of an interference, definitely embodied by the avatars. 
As a counterpart of this, we observe that:

- the sign difference is irrelevant, since at times the contribution of the interference is positive and at other times negative, as evidenced by the double slit pattern of Figure 31, and

- in one case it is about entropies, and in the other about probabilities, however, the identical morphology of the equations shows a phenomenon of similar characteristics.
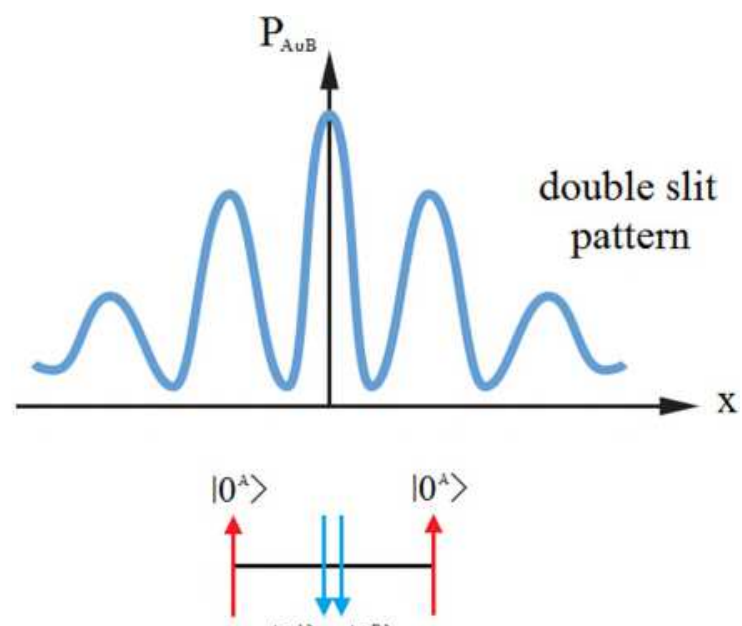

$\left|1^{A}\right\rangle\left|1^{B}\right\rangle$

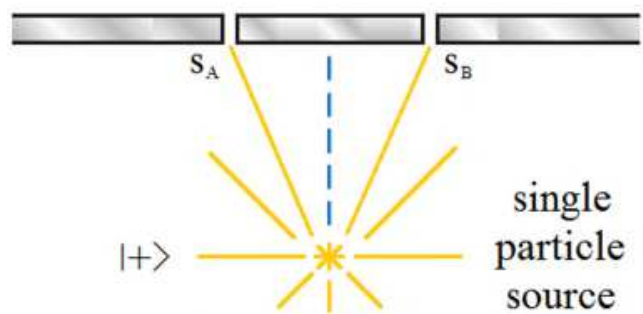

Figure 31. Double slit experiment with both slits open.

At this point, it is interesting to ask ourselves, how is it possible that an entanglement arises from a double slit experiment? To answer this question, we are going to make two assumptions:

a) the single particle source generates particles instead of waves as in the case of Equation 76. Using the same criteria of Equation 76, the particles will be of the form: $|+\rangle=(|0\rangle+|1\rangle) / \sqrt{2}$, where $|+\rangle$ is equivalent to $\psi_{A \cup B}(x),|0\rangle$ is equivalent to $\psi_{A}(x),|1\rangle$ is equivalent to $\psi_{B}(x)$, and $c=1 / \sqrt{2}$, and 
b) the double slit assembly is a clone machine, which tries to clone the incident particle to the double slit assembly into two identical ones.

The problem is that when we try to apply a universal cloning machine to different qubits, the following happens ${ }^{[64]}$ :

- $\quad|0\rangle \rightarrow[$ copy machine $] \rightarrow|0\rangle|0\rangle$,

- $\quad|1\rangle \rightarrow[$ copy machine $] \rightarrow|1\rangle|1\rangle$, and

- $\quad|+\rangle=(|0\rangle+|1\rangle) / \sqrt{2} \rightarrow[$ copy machine $] \rightarrow(|00\rangle+|11\rangle) / \sqrt{2}=\left|\beta_{00}\right\rangle$.

That is, in those cases where the qubits that are being cloned have a projection exclusively on a single axis of the Bloch sphere, as is the case of the CBS $\{|0\rangle,|1\rangle\}$, the copying machine works perfectly ${ }^{[65]}$, but in those other cases involving superposition, the double-slit assembly is a lousy cloning machine, that is the reason why it generates $\left|\beta_{00}\right\rangle$ from $|+\rangle$. This explains the appearance of the entanglement in the double-slit experiment.

Finally, if we apply a detector in any of the slits, equivalent to a quantum measurement in the entanglement, we arrive at Figure 32, which corresponds to the case of completely independent
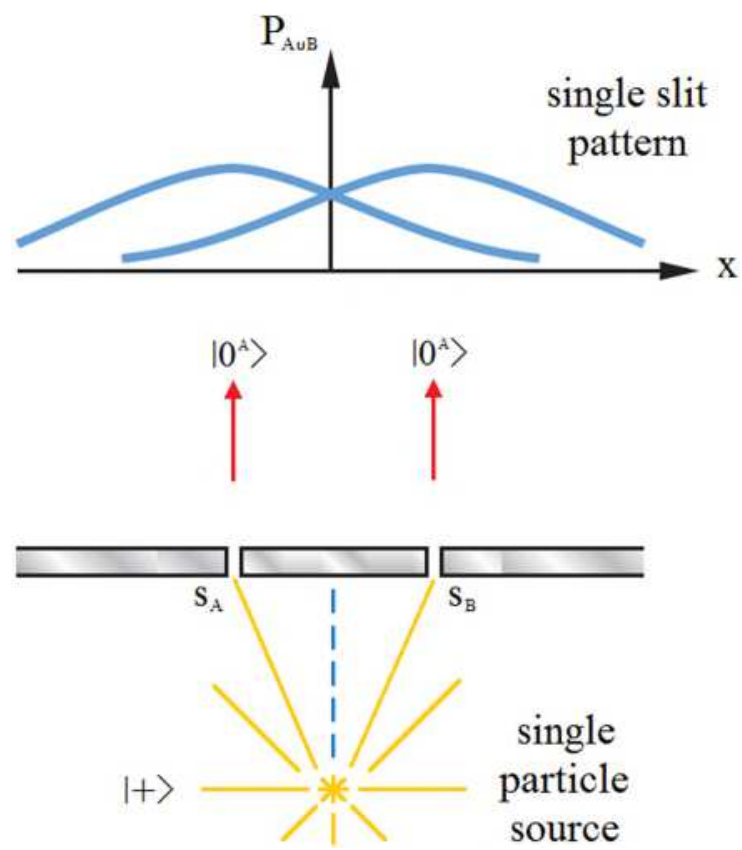

Figure 32. Double slit experiment with only one slit open each time. 
particles, where the absence of avatars in entanglement is completely equivalent to that of interference in the double-slit experiment.

To summarize, the impossibility of making a perfect copy gives rise to the entanglement and the interference pattern in the double-slit experiment, which is equivalent to saying that the absence of a perfect copy machine is the root of these extraordinary phenomena. It is not surprising that the most interesting things in Quantum Physics revolve around the No-Cloning Theorem. ${ }^{[18]}$

\subsection{Times analysis}

As an example that allows us to make it clear what is instantaneous and what is not in a communication system that uses entanglement based on the concepts developed so far, in this subsection we analyze all the times involved in the full 4-bit transmission for a configuration like the one in Figure 11. Imagine that we will transmit those 4 bits from Earth to Mars and that we will send the entangled photon or EPR to Mars through a double telescope optical system. ${ }^{[42]}$ As we will see below, no communication system based on entanglement can take place in real time due to the time it takes for light to travel the distance between Earth and Mars: 4, 10, or 20 minutes, which depends on the distance between both planets at a particular time.

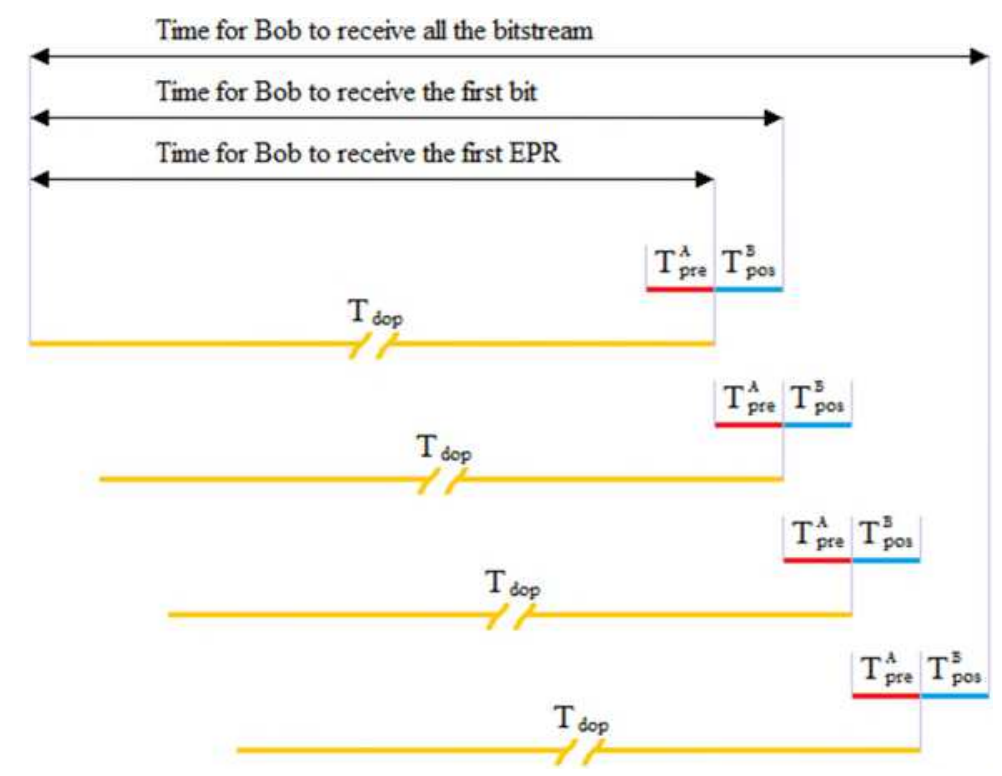

Figure 33. Times involved in the transmission of 4 bits using the configuration of Figure 11. 
Assuming that some of the times involved in this example can be overlapped, the full (absolute) time for the transmission of the first bit of Figure 33 will be:

$T_{a 1 b}=T_{s v e}+T_{d o p}+T_{p o s}^{B}$,

where,

$T_{a 1 b}:$ absolute (total) time for the transmission of one bit, e.g., the first one,

$T_{\text {sve }}:$ synchronization time via entanglement $=$ effect time or entanglement time $=0$,

$T_{d o p}:$ time for the distribution of every entangled pair or EPR pair,

$T_{\text {pos }}^{B}$ : postprocessing time of Bob's side $=$ transit time through the gates on Bob's side + transit time through the Quantum-to-Classical (Q2C) interface (measurement, or state tomography) + the time for the delivery of the information.

At this point, we make five important assumptions, which do not alter the balance of times involved at all:

1. the transmission system begins with the previous distribution of the first EPR pair by Alice, as well as the rest of the consecutive entangled pairs, before any type of formal information transfer takes place between Alice and Bob,

2. Alice retains her entangled photon in a quantum memory ${ }^{[53,66]}$,

3. Alice begins with the process of acquiring and processing the first bit to transmit before the first EPR arrives at Bob's side, that is, part of the travel time of the EPR pair from Alice to Bob overlaps with the preprocessing time of the bit to be transmitted by Alice, which is memory intensive in order to compensate for any timing differences in the above times,

4. the entangled photon (EPR) sent from Earth by Alice, reaches Bob on Mars without the need for any additional satellite configuration that performs optical relay tasks to complement the aforementioned double telescope system, i.e., we assume a perfect optical transmission without any type of interference, and 
5. $T_{p r e}^{A} ; T_{p o s}^{B}$, where, $T_{p r e}^{A}$ : preprocessing time of Alice's side = acquisition time of the bits to transmit + transit time through the Classical-to-Quantum (C2Q) interface + transit time through the gates on Alice's side.

Since the synchronization time via entanglement $T_{s v e}$, effect time, or entanglement time is 0 , Equation 81 results in,

$T_{a 1 b}=T_{d o p}+T_{p o s}^{B}$

In all practical cases, including the present case of transmission of 4 bits from Earth to Mars, the distribution time of the entangled pair, which generates the quantum channel, is the longest time involved in any communication system that uses entanglement, that is,

$T_{d o p} ? T_{p o s}^{B}$

Then,

$T_{a 1 b} ; T_{d o p}$

Consequently, if we look at Figure 33, we can overlap the distribution times of the subsequent entangled pairs so that the transmitted stream does not lose the desired continuity. Therefore, the time required to receive the full 4-bit stream (see Figure 33) will be:

$T_{a 4 b}=T_{d o p}+4 T_{p o s}^{B}$

where,

$T_{a 4 b}:$ absolute (total) time for the transmission of the 4 bits (complete stream).

At this point in our analysis, even if it is a case in which the transmission is exclusively unidirectional with extremely large bitstream, perhaps for remote storage, or in order to transfer instructions to an individual or system, in which case $T_{d o p}=N T_{p o s}^{B}$, the time wasted in the distribution of the entangled pair is not amortized here either. The transmission time performance of the aforementioned bitstream is very low, which is an indication of how unprofitable or inefficient a transmission system based on entanglement is for the case of long 
distances, in fact, the transmission time efficiency is the same as that of a purely classical equivalent system. The only thing that justifies the migration to a quantum system concerns security, this being the reason why for the future migration of the current Internet to its quantum version. Even in a more bidirectional context, even though the stream has continuity and this implies a very large number of bits to transmit, the distribution time of the EPR pairs occupies an excessive percentage of the total transmission time. Besides, the bi-directionality itself must be added, with which everything starts over, every time we say over.

On the other hand, heavy use of quantum memories allows us to put preprocessing time on Alice's side $T_{p r e}^{A}$ in the shadow of time for the distribution of every entangled pair (or EPR) $T_{d o p}$. This is highly recommended, in order to save some time. In this context, the only thing that can interrupt the stream is a difficulty in the bit acquisition on Alice's side. Regardless of the context, the distribution time $T_{d o p}$ is impossible to eliminate.

What the literature treats as instantaneity of entanglement, which can eventually be exploited in a quantum communication system, concerns exclusively the process that takes place after the distribution of the entangled pair has been carried out. Regardless of the size of the bit-stream to be transmitted, the obvious and necessary bi-directionality of any communication system forces everything to start over again by changing the role of each participant from receiver to sender, thus eliminating eventual progress in the continuity of the bit-stream transmission.

It is precisely this conclusion that this study tries to reach, trying to establish definitively that an instantaneity of the process that takes place after the distribution of the entangled pairs; be it without control, with quasi-control, or with complete local control of non-local outcomes; does not imply at all the fact that the communication system that uses the entanglement is instantaneous, precisely because of the initial relativistic time for the distribution of the entangled pair, which gives rise to the essential quantum channel.

\section{Conclusions}


An internal modeling of the PBS was presented in Section 3 based on a horizontal and vertical polarizers $\left\{\mathrm{P}_{\mathrm{H}}, \mathrm{P}_{\mathrm{V}}\right\}$. Thanks to this modeling, it can be understood how the quantum measurement operator $M$ works, which acts as a closed system without access from the outside, i.e., outside of our external control. In particular, the PBS model was used to measure the Bell state $\left|\beta_{00}\right\rangle$ at the $\{|H\rangle,|V\rangle\}$ basis, although the model is perfectly extensible to the $\{|D\rangle,|A\rangle\}$ basis (Equation 5) by simply replacing the polarizers $\left\{\mathrm{P}_{\mathrm{H}}, \mathrm{P}_{\mathrm{V}}\right\}$ by $\left\{P_{D}, P_{A}\right\}=\{|D\rangle\langle D|| A\rangle,\langle A|\}$, or to the $\{|R\rangle,|L\rangle\}$ basis (Equation 6), thanks to the polarizers $\left\{P_{R}, P_{L}\right\}=\{|R\rangle\langle R|| L\rangle,\langle L|\}$, even for the remaining Bell states of Equation 28.

Moreover, an analogy between the randomness of quantum measurement process with the toss of a coin by different actors was established, where we associate the result "head" with the activation of the horizontal polarizer $\mathrm{P}_{\mathrm{H}}$ and the deactivation of the vertical polarizer $\mathrm{P}_{\mathrm{V}}$, while the result "tail" is associated to the opposite. Said process is clearly of the stochastic type, where all quantum measurement results from an average of the outcomes obtained in each case, however, the instantaneous synchronization of results takes place without the use of a classical channel. Curiously, part of this stochastic process is deterministic, since after the coin is tossed, each coin result will produce the same output: $\{$ head $\rightarrow|H\rangle \equiv|0\rangle$, tail $\rightarrow|V\rangle \equiv|1\rangle\}$.

In Section 4, we use the same PBS model but as an open system, i.e., under our complete control, associated with an optical experiment consisting of a rotatable polarizer, for only two positions $\{\mathrm{H}, \mathrm{V}\} \equiv\left\{0^{\circ}, 90^{\circ}\right\}$, controlled by a computer, which when emitting a 0 activates the horizontal polarizer $\mathrm{P}_{\mathrm{H}}$ and deactivates the vertical $\mathrm{P}_{\mathrm{V}}$, while when emitting a 1, the opposite happens. The possibility of locally controlling non-local outcomes through the local application of a $\mathrm{P}_{\mathrm{H}}$ vertical polarizer (Figure 14) made the experiment completely deterministic, and without the use of a classical channel. The probabilities mentioned in this section have to do with those that unpolarized light like $\left|\beta_{00}\right\rangle$ has of crossing a horizontal polarizer $\mathrm{P}_{\mathrm{H}}$ or a vertical polarizer $\mathrm{P}_{\mathrm{V}}$, 
which are always $50 \%$.

The total compatibility between the model of a PBS and that of the aforementioned rotatable polarizer acting on $\left|\beta_{00}\right\rangle$ in the $\{|H\rangle,|V\rangle\}$ basis, is the main responsible for the local control of non-local results in an instantaneous way, since, both the PBS and the rotatable polarizer act on the same Bell state, with the same model and on the same basis, in one case without external control and in the other one with external control, respectively.

Such is the degree of identity of both models that regardless of whether or not man can take advantage of post-distribution instantaneity to send information or not, it is clearly established that the conflict between post-distribution instantaneity and the No-Cloning Theorem present in literature ${ }^{[19,20]}$ is not something inherent to Nature but rather to the configuration of the protocols used to date. In fact, the PBS model (identical to that of the rotatable model) shows Nature instantaneously synchronizes long distance results, and said instantaneity is established per se in the entanglement locally controlling non-local outcomes. Then, if Nature can achieve it with the same model, why not man?

In Section 5, we analyze the resulting percentage of outcomes obtained by the intervention of the polarizers $\left\{\mathrm{P}_{\mathrm{H}}, \mathrm{P}_{\mathrm{V}}\right\}$, and we introduce the possibility of increasing this percentage through an amplification process. Said amplifier is only viable in specific cases ${ }^{[18]}$, and even more so in the case of CBS $\{|0\rangle,|1\rangle\}$, which can be cloned ${ }^{[63]}$, and therefore amplified without any problem. Moreover, both the PBS model as well as the rotatable polarizer model proved to have excellent performance when applied to the quantum teleportation protocol, adjusting perfectly to the experimental probabilities of the outcomes, in the first case, and accurately predicting the value of the outcomes, in the second case, until concluding in the equivalent (simplified) circuit of Figure 18, which showed the same efficiency for two practical examples implemented on Quirk simulator. ${ }^{[46]}$ The same performance was obtained, with and without amplification factor, when applying the simplified model to three of the main tools in the quantum Internet toolbox: 
quantum secret sharing, entanglement swapping, and quantum repeater. In all cases, a simplified configuration based on $\mathrm{P}_{\mathrm{H}}$ made it possible to convert all these protocols into deterministic and without the need for classical channels.

Section 6 begins with an analysis of the results of the previous sections, and continues with an answer to the question that has been present in Physics for the last hundred years: how is it possible that a random phenomenon can be instantaneously synchronized, and independently of the distance that separates the particles involved in said phenomenon? The answer involved a new internal model of entanglement, as well as a new analysis about randomness and instantaneity in the synchronization of non-local outcomes as a result of a quantum measurement; without confronting two of the main pillars of Physics: Special Relativity, and Quantum Mechanics.

The entanglement model presented here shows us that:

- unlike the independent and classically-correlated cases, the entanglement constitutes a unified and dynamic structure, with a rotating axis that links the original particles and the avatars, where the instantaneous synchronization is due to the rotation of the unified structure and not to a faster-than-light (FTL) transmission of a synchronization order between $\mathrm{A}$ and $\mathrm{B}$, which eliminates any confrontation between two of the main pillars of Physics: Special Relativity and Quantum Mechanics,

- the localities of the particles overlap forming a single and extended locality which is equivalent to a non-locality, and

- as a consequence of the above, there is no channel between $\mathrm{A}$ and $\mathrm{B}$, no transmitter, and no receiver. In fact, once the entangled pair is distributed, as there is no channel between $\mathrm{A}$ and $\mathrm{B}$, nothing in the middle of Alice and Bob interrupts this mechanism, delays or attenuates it, without the need to use antennas or power system to amplify an eventual signal to be transmitted. For the entanglement, it is as if the entire universe was no more than a point, that point where the avatars are. 
In the literature, it is accepted in a per se way, that for there to be randomness in the result of an entanglement measurement it is because quantum Nature is inherently random. However, if we add the PBS model with the entanglement model seen in this work, we can attack randomness in another way, i.e., dividing the problem into parts in order to trace the true factor responsible for such randomness.

In fact, both the PBS and the entanglement could have perfectly regular clocks, and both clocks could even be synchronized to such an extent that they are the same clock (with the same frequency and perfectly in-phase) and even then the randomness would be ensured by the classical (non-quantum) intervention of man by getting the PBS in the way of the Bell state at a certain point in time.

This is mainly due to two factors:

- this moment is impossible to establish exactly by man with the intention of making the two elements work in perfect synchronization or in phase (between the internal clock of the PBS or entanglement and the moment of applying the PBS), given the impossibility that the experimenter has to access in practice the internal process that takes place in the PBS or the entanglement itself to synchronize the application of the PBS with a particular instant of the internal clock, and

- the macroscopic (classical) scale in time and space of human action to locate the PBS in the path of the entangled photon contrasts so extravagantly with the frequency of the internal PBS clock and entanglement, that the result could not be other than absolutely random

In other words, there could be a single clock (equal frequency and phase) that synchronizes everything in the quantum universe and still the quantum measurement turns out to be random as a whole due to human intervention from the classical world. Consequently, we conjectured that God does not play dice, that is, in terms of the existence of a clock with which everything in the quantum universe is organized, something like a metronome of the quantum universe rhythm. 
The intrinsic randomness raised by the literature is less understandable, more complex, and further from the typical quantum characteristics where everything is so organized and regular. Therefore, applying the Ockham's razor ${ }^{[58,59]}$ : pluralitas non-est ponenda sine necessitate (plurality should not be posited without necessity) we ask ourselves: why complicate things with an independence and concurrence of two randomness, if instead everything can be at the same frequency and in phase by means of a single clock in the quantum world and still explain the randomness of the quantum measurement completely? To such an extent, the randomness analysis presented in this work is more satisfactory to us since it is the only one of the two criteria (that of the literature and this one) that can explain why in the face of an ambiguity the quantum nature is organized by octaves to generate the options necessary in Figures 19, 20, 23(b), 24(b), and 25(b), maintaining a basal rate consisting of the amount of ones per second. Moreover, a homology between the entanglement and the double slit experiment is presented. This homology fits exactly in both cases: the double slit and the entanglement, as well as in the case of the single slit and completely independent particles, which shows that entanglement occurs in the double slit experiment and, at the same time, there is always an interference inside entanglement, which is embodied by the avatars.

Section 6 concluded with the time analysis of a communication system based on entanglement, showing that the greatest time involved in the transmission of each bit is the distribution of the entangled pair (quantum channel), which is irreducible and much greater than zero, therefore, regardless of the control and instantaneity that the post-distribution process of the EPR pairs may have, a communication system based on entanglement can never be instantaneous.

Entanglement is a phenomenon with the following characteristics: it is

- non-local,

- instant in post-distribution, and

- controllable without a classical channel with the appropriate experiment. 
Emphasizing the second characteristic, the three experiments in Sections 3-5 show that the postdistribution time can be zero, in the absence of a classical channel, and yet the communication system, as a whole, will never be instantaneous.

Moreover, entanglement is not a communication system per se, and an entanglement-based communication system must necessarily include the pre-distribution of entangled particles to generate a quantum channel, which happens at relativistic speeds. Therefore, although entanglement was instantaneous with respect to a remote control of non-local outcomes through local actions, the transmission of information through such a system could never be instantaneous due to successive distributions of entangled pairs at great distances at relativistic speeds, since for the transfer of each qubit to complete, we must first remove said qubit from the transmitter in order not to violate the No-Cloning Theorem. That is, it is the distribution of the entangled pairs that prevents the instantaneity of a communication system based on entanglement and nothing else, which confirms what is established in this study.

In fact, the transmission of a given qubit would never take place if the EPR is not first distributed in relativistic times, which makes the instantaneous transmission of the qubit impossible.

To summarize, a communication system based on entanglement necessarily includes the distribution of entangled pairs, ergo such a system can never be instantaneous, this being exclusively the reason for non-instantaneousness, and not any other.

\section{Data Availability}

The experimental data that support the findings of this study are available in ResearchGate with the identifier https://doi.org/10.13140/RG.2.2.36266.13766.

\section{Acknowledgements}

M. Mastriani thanks the staff of the Knight Foundation School of Computing and Information Sciences at Florida International University for all their help and support.

\section{Conflict of Interest}

The author declares no conflict of interest. 


\section{References}

[1] J. Audretsch, Entangled Systems: New Directions in Quantum Physics, Wiley-VCH Verlag GmbH \& Co., Weinheim, Germany 2007.

[2] G. Jaeger, Entanglement, Information, and the Interpretation of Quantum Mechanics, The Frontiers Collection, Springer-Verlag, Berlin, Germany 2009.

[3] R. Horodecki, P. Horodecki, M. Horodecki, K. Horodecki, Rev. Mod. Phys. 2009, 81, 865.

[4] A. Einstein, Annln. Phys. 1911, 35, 898; Reprinted in A. Einstein, H. A. Lorentz, H. Minkowski, H. Weyl, The Principle of Relativity: a collection of original memoirs on the special and general theory of relativity, Methuen, London, UK 1923, reprinted by Courier Dover Publications, New York, USA 1952.

[5] A. C. Phillips, Introduction to Quantum Mechanics, Wiley, Chichester, UK 2003.

[6] A. Einstein, B. Podolsky, N. Rosen, Phys. Rev. 1935, 47, 10, 777.

[7] A. Einstein, in Albert Einstein, Philosopher Scientist, edited by P. A. Schilpp, Evanston, Library of Living Philosophers III, 85, Illinois, USA 1949.

[8] A. Shimony, Controllable and uncontrollable nonlocality, in S. Kamefuchi et al., ed., Proc. of the Inter. Symp. on the Foundations of Quantum Mechanics, Tokyo, Physical Society of Japan, 225, (1984).

[9] J. Bell, Physics 1964, 1, 195.

[10] J. F. Clauser, M. A. Horne, A. Shimony, R. A. Holt, Phys. Rev. Lett. 1969, 23, 15, 880.

[11] A. Aspect, P. Grangier, G. Roger, Phys. Rev. Lett. 1982, 49, $2,91$.

[12] A. Aspect, J. Dalibard, G. Roger, Phys. Rev. Lett. 1982, 49, 25, 1804.

[13] A. Aspect, Nature 2007, 446, 7138, 866.

[14] R. Hanson, Nature 2015, 526, 682. 
[15] C. H. Bennett, G. Brassard, C. Crépeau, R. Jozsa, A. Peres, W. K. Wootters. Phys. Rev. Lett. 1993, 70, 1895.

[16] D. Bouwmeester, J-W. Pan, K. Mattle, M. Eibl, H. Weinfurter, A. Zeilinger, Nature 1997, 390, 575 .

[17] D. Boschi, S. Branca, F. De Martini, L. Hardy, S. Popescu, Phys. Rev. Lett. 1998, 80, 1121.

[18] W. K. Wootters, W. H. Zurek, Nature 1982, 299, 802.

[19] K. Banaszek, R. Demkowicz-Dobrzanski, Quantum information 1/2, https://www.fuw.edu.pl/ demko/Teksty/ik05/2012/qi12.pdf, 71, June, 2012.

[20] M. Krenn, M. Malik, T. Scheidl, R. Ursin, A. Zeilinger, Quantum Communication with Photons, in: Al-Amri M., El-Gomati M., Zubairy M. (eds) Optics in Our Time, 460, Springer, Cham, Switzerland 2016.

[21] .-, Lecture Notes in Physics 797: Applied Quantum Cryptography. C. Kollmitzer and M. Pivk Eds, Heidelberg, Springer, 2010.

[22] A. Ekert, Quantum Cryptography, in A.V. Sergienko (Ed.): Quantum Communications and Cryptography: Optical Science and Engineering, CRC Press, Boca Raton, USA 2006.

[23] M. Caleffi, D. Chandra, D. Cuomo, S. Hassanpour, A. Cacciapuoti, Computer 2020, $53,06,67$.

[24] D. Chandra, S. A. Cacciapuoti, M. Caleffi, L. Hanzo, arXiv, preprint, https://arxiv.org/abs/2012.11982, December, 2020.

[25] A. S. Cacciapuoti, M. Caleffi, F. Tafuri, F. S. Cataliotti, S. Gherardini, G. Bianchi, IEEE Network 2020, 34, 1, 137.

[26] A. S. Cacciapuoti, M. Caleffi, R. van Meter, L. Hanzo, IEEE Trans. on Comm. 2020, $68,6,3808$.

[27] M. Caleffi, A. S. Cacciapuoti, IEEE Journal on Selected Areas in Communications 2020, $38,3,575$. 
[28] M. Caleffi, A. S. Cacciapuoti, G. Bianchi, Quantum Internet: from Communication to Distributed Computing! NANOCOM'18: Proc. 5th ACM Int. Confe. on Nanoscale Comp. \& Comm., Reykjavik, Iceland, 1 (Sept., 2018).

[29] D. Cuomo, M. Caleffi, A. S. Cacciapuoti, IET Quantum Communication 2020, 1, 1, 3.

[30] K. Chakraborty, F. Rozpedeky, A. Dahlbergz, S. Wehner, arXiv, preprint, https://arxiv.org/abs/1907.11630, July, 2019.

[31] S. Wehner, D. Elkouss, R. Hanson, Science 2018, 362, eaam9288.

[32] W. Dür, R. Lamprecht, S. Heusler, Eur. J. Phys. 2017, 38, 043001.

[33] H. J. Kimble, Nature 2008, 453, 1023.

[34] L. Gyongyosi, S. Imre, Quant. Info. Proc. 2020, 19, 115.

[35] L. Gyongyosi, S. Imre, arXiv, preprint, https://arxiv.org/abs/1905.00256, May, 2019.

[36] L. Gyongyosi, S. Imre, arXiv, preprint, https://arxiv.org/abs/1905.00258, May, 2019.

[37] M. A. Nielsen, I. L. Chuang, Quantum Computation and Quantum Information, Cambridge University Press, Cambridge, UK 2004.

[38] P. Busch, P. Lahti, J. P. Pellonpää, K. Ylinen, Quantum Measurement. Springer, A.G. Switzerland 2016.

[39] A. Furusawa, P. van Loock, Quantum Teleportation and Entanglement: A Hybrid Approach to Optical Quantum Information Processing, Wyley-VCH, Weinheim, Germany, 2011.

[40] M. Sadiq, Doctor of Philosophy in Physics, Stockholm University, March, 2016.

[41] D. F. V. James, P. G. Kwiat, Quantum State Entanglement Creation, characterization, and application. Los Alamos Science, 27, 52, https://permalink.lanl.gov/object/tr?what=info:lanl-repo/lareport/LA-UR-02-1236, August, 2006.

[42] A. Zeilinger, Phys. Scr. 2017, 92, 072501. 
[43] G. Cariolaro, Quantum Communications: Signals and Communication Technology, Springer, A. G., Switzerland 2015.

[44] S. Imre, L. Gyongyosi, Advanced Quantum Communications: An Engineering Approach, Wiley-IEEE Press, N.Y., USA 2013.

[45] M. Benslama, A. Benslama, S. Aris, Quantum Communications in New Telecommunications Systems. John Wiley \& Sons, Hoboken, USA 2017.

[46] Algorithmic Assertions. https://algassert.com/quirk, accessed: March, 2021.

[47] D. Joy, M. Sabir, B. K. Behera, P. K. Panigrahi, Quantum Inf. Process. 2020, 19, 33.

[48] J-W. Pan, Z-B. Chen, C-Y. Lu, H. Weinfurter, A. Zeilinger, M. Zukowski, Rev. Mod. Phys. 2012, 84, 777.

[49] N. Sangouard, C. Simon, H. de Riedmatten, N. Gisin, Rev Mod Phys. 2011, 83, 33.

[50] Y. Hasegawa, R. Ikuta, N. Matsuda, K. Tamaki, H-K. Lo, T. Yamamoto, K. Azuma, N. Imoto, Nat Commun. 2019, 10, 378.

[51] W. J. Munro, K. Azuma, K. Tamaki, K. Nemoto, IEEE J. Sel. Top Quantum Electron. 2015, 21, 6400813.

[52] Q. Ruihong, M. Ying, IOP J. Phys. Conf. Ser. 2019, 1237, 052032.

[53] M. Razavi, M. Piani, N. Lutkenhaus, arXiv, preprint, https://arxiv.org/abs/0810.5334, September, 2009.

[54] K. Boone, J.-P. Bourgoin, E. Meyer-Scott, K. Heshami, T. Jennewein, C. Simon, Phys. Rev. A 2015, 91, 5, 052325.

[55] M. Mastriani, S. S. Iyengar, Wiley, Quantum Engineering, QUE255 2020.

[56] M. Mastriani, S. S. Iyengar, K.J. Latesh Kumar, Quantum Inf. Process. 2021, 20, 22.

[57] M. Mastriani, S. S. Iyengar, K.J. Latesh Kumar, Satellite quantum communication protocol regardless of the weather, accepted in Optical and Quantum Electronics 2021.

[58] W. M. Thorburn, Occam's razor, Mind 1915, 24, 287-288.

[59] W. M. Thorburn, The Myth of Occam's razor, Mind 1918, 27, 345. 
[60] M. Schlosshauer, Reviews of Modern Physics 2005, 76, 4, 1267.

[61] H. P. Hsu, Fourier Analysis. Simon \& Schuster, New York, USA 1970.

[62] D. J. C. MacKay, Information Theory, Inference, and Learning Algorithms, Cambridge University Press, Cambridge, UK 2003.

[63] A. Ananthaswamy, Through Two Doors at Once: The Elegant Experiment That Captures the Enigma of Our Quantum Reality, Dutton Books, NY, USA 2018.

[64] W. Dür, S. Heuslery, arXiv, preprint, https://arxiv.org/abs/1312.1463, December, 2013.

[65] A. J. Arul, arXiv, preprint, https://arxiv.org/abs/quant-ph/0107085, July, 2001.

[66] A. Lvovsky, B. Sanders, W. Tittel, Nature Photon 2009, 3, 706. 